\title{
Uniform Asymptotic Expansions for Weber Parabolic Cylinder Functions of Large Orders
}

\author{
F. W. J. Olver*
}

(April 6, 1959)

Expansions of solutions of the differential equation

$$
\frac{d^{2} w}{d t^{2}}=\mu^{4}\left(t^{2}-1\right) w,
$$

are sought for large values of $|\mu|$, which are uniformly valid with respect to arg $\mu$ and unrestricted values of the complex variable $t$. Two types of expansion are found. Those of the first type are in terms of elementary functions and are valid outside the neighborhoods of the points $t= \pm 1$, the turning points of the differential equation. The second are in terms of Airy functions and hold in unbounded regions containing one of the turning points.

The special forms of the expansions when the variables are real are considered in detail, and asymptotic expansions for the zeros of solutions of the differential equation are found by reversion. Numerical examples are included.

\section{Contents}

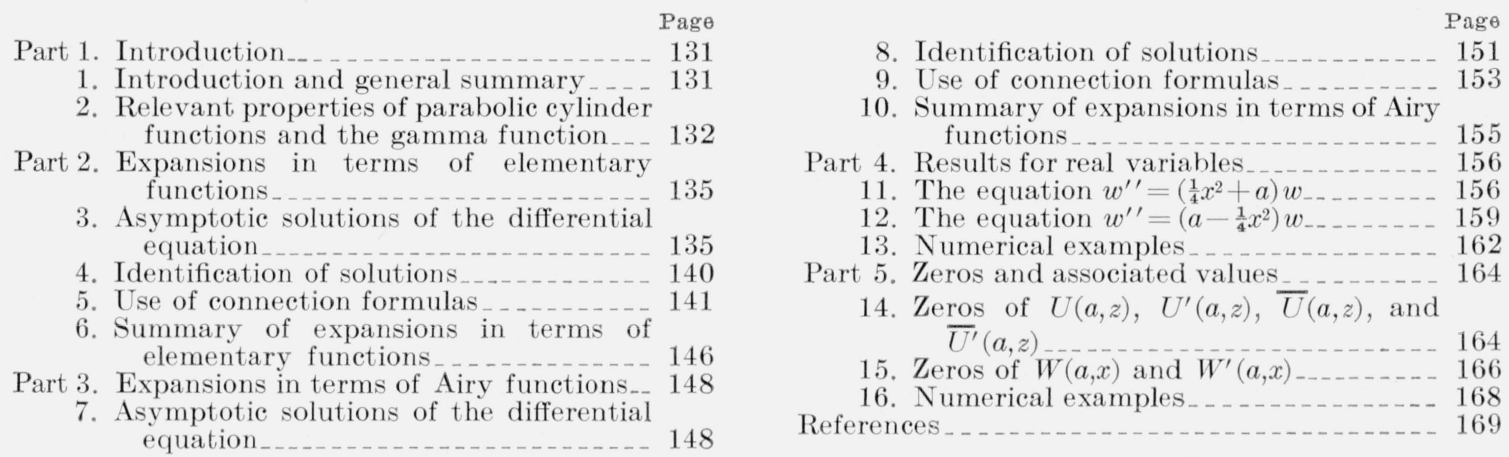

\section{Part 1. Introduction}

\section{Introduction and General Summary}

The purpose of this paper is to determine asymptotic expansions of solutions of the differential equation

$$
\frac{d^{2} w}{d t^{2}}=\mu^{4}\left(t^{2}-1\right) w
$$

for large values of the complex parameter $\mu$ which are uniformly valid with respect to arg $\mu$ and the complex variable $t$.

The immediate reason for this investigation is that the solutions of eq (1.1) are basic functions in the theory of the asymptotic solution of the differential equation

$$
\frac{d^{2} w}{d t^{2}}=\left\{\mu^{4} p(t)+q(t)\right\} w
$$

for large values of $|\mu|$ in a region in which $p(t)$ has two simple zeros, just as Airy functions are

\footnotetext{
*Present address: National Physical Laboratory, Teddington, Middlesex, England.
} 
basic functions in the theory of eq (1.2) in a region containing one simple zero of $p(t)$. A knowledge of the uniform asymptotic behavior of the solutions of (1.1) is required in developing the asymptotic theory of (1.2).

Equation (1.1) is of interest, however, in other connections, for example in the study of wave motion inside or outside parabolic cylinders, and wave propagation in an inhomogeneous atmosphere. Hermite polynomials of order $n$ are expressible in terms of the solutions of the equation when $\mu^{2}=2 n+1$. Accordingly, for the sake of completeness many results are derived in this paper which go beyond those needed for the immediate purpose stated in the preceding paragraph.

The desired asymptotic expansions are obtained by application of the general theory of the asymptotic solution of linear differential equations of the second order as developed by the present writer in [9]. ${ }^{1}$ From the standpoint of the general theory, eq (1.1) is characterized by having turning points at $t= \pm 1$. Asymptotic expansions in terms of elementary functions can be found in any region bounded away from these points and expansions of this kind are obtained in part 2 by application of theorem $\mathrm{A}$ of [9].

In order to determine the behavior near the turning points, expansions in terms of Airy functions need to be used. In part 3 these expansions are obtained by application of theorem $\mathrm{B}$ of [9]; they are valid in unbounded $t$-regions which include one (but not both) of the turning points. All combinations of $\mu$ and $t$ are covered.

When the variables are real, eq (1.1) has important special solutions. Expansions of these solutions are derived in part 4 from the results of parts 2 and 3 . Also included in part 4 are numerical examples illustrating the powerful nature of the expansions.

In part 5 uniform asymptotic expansions for the zeros and associated values are obtained by reversion and illustrated by numerical examples. Much of the analysis of this part and some in earlier parts is similar to that used in [8] in the theory of Bessel functions of large order, and advantage is taken of this analogy whenever possible.

Investigations of the asymptotic solution of eq (1.1) for large $|\mu|$ have been made by several writers. Their work on expansions in terms of elementary functions is described in section 6 of part 2, and on expansions in terms of Airy functions in section 10 of part 3 . At this stage it suffices to remark that the expansions obtained in this paper include practically all of the earlier results and extend them in various ways.

\section{Relevant Properties of Parabolic Cylinder Functions and the Gamma Function}

A comprehensive account of the properties of parabolic cylinder functions is given by Miller in the Introduction to [4]. For the purpose of reference we collect here the properties which will be required in this paper. We use Miller's notation rather than that of Whittaker [13] and adopt Miller's choice of solutions when the variables are real.

The standard form of differential equation for the parabolic cylinder functions is

$$
\frac{d^{2} w}{d z^{2}}=\left(\frac{1}{4} z^{2}+a\right) w
$$

The principal solution $U(a, z)$ is determined by the condition

$$
U(a, z) \sim z^{-a-\frac{1}{2}} e^{-\frac{1}{4} z^{2}} \quad \text { as } z \rightarrow+\infty .
$$

It is an integral (entire) function of $z$ and an integral function of $a$. In terms of Whittaker's notation $D_{n}(z)$ for parabolic cylinder functions and $W_{k, m}(z)$ for the confluent hypergeometric function, we have

$$
U(a, z)=D_{-a-\frac{1}{2}}(z)=2^{-\frac{1}{2} a} z^{-\frac{1}{2}} W_{-\frac{1}{2} a,-\frac{1}{4}}\left(\frac{1}{2} z^{2}\right) .
$$

\footnotetext{
${ }^{1}$ Figures in brackets indicate the literature references at the end of this paper.
} 
Other solutions of $(2.1)$ are $U(a,-z), U(-a, i z)$ and $U(-a,-i z)$. The connection formulas are

$$
\begin{gathered}
U(-a, \pm i z)=(2 \pi)^{-\frac{1}{2}} \Gamma\left(\frac{1}{2}+a\right)\left\{e^{-i \pi\left(\frac{1}{2} a-\frac{1}{4}\right)} U(a, \pm z)+e^{i \pi\left(\frac{1}{2} a-\frac{1}{4}\right)} U(a, \mp z)\right\}, \\
U(a, \pm z)=(2 \pi)^{-\frac{1}{2}} \Gamma\left(\frac{1}{2}-a\right)\left\{e^{-i \pi\left(\frac{1}{2} a+\frac{1}{4}\right)} U(-a, \pm i z)+e^{i \pi\left(\frac{1}{2} a+\frac{1}{4}\right)} U(-a, \mp i z)\right\} .
\end{gathered}
$$

For fixed $a$ and large $|z|$

$$
U(a, z)=z^{-a-\frac{1}{2}} e^{-\frac{1}{4} z^{2}}\left\{1+O\left(|z|^{-2}\right)\right\} \quad\left(|\arg z| \leq \frac{3}{4} \pi-\epsilon\right),
$$

where $^{2}$ the $O$ is uniform with respect to arg $z$. Here and elsewhere $\epsilon$ denotes an arbitrary positive number which is independent of all other variables. From (2.4) and (2.6), we obtain

$$
U(a,-z)=e^{-i \pi\left(a+\frac{1}{2}\right)} z^{-a-\frac{1}{2}} e^{-\frac{1}{4} z^{2}}\left\{1+O\left(|z|^{-2}\right)\right\}+\frac{(2 \pi)^{\frac{1}{2}}}{\Gamma\left(\frac{1}{2}+a\right)} z^{a-\frac{1}{2}} e^{\frac{1}{4} z^{2}}\left\{1+O\left(|z|^{-2}\right)\right\},
$$

valid when $-\frac{3}{4} \pi+\epsilon \leq \arg z \leq \frac{1}{4} \pi-\epsilon$. The same result with the sign of $i$ changed holds when $-\frac{1}{4} \pi+\epsilon \leq \arg z \leq \frac{3}{4} \pi-\epsilon$; the apparent discrepancy between the formulas in their common region of validity is merely an example of the Stokes phenomenon.

Let the z-plane be divided into four sectors $\mathbf{M}_{0}, \mathbf{M}_{1}, \mathbf{M}_{2}$, and $\mathbf{M}_{3}$, defined by $|\arg z| \leq \frac{1}{4} \pi$, $\frac{1}{4} \pi \leq \arg z \leq \frac{3}{4} \pi, \quad|\arg (-z)| \leq \frac{1}{4} \pi$ and $-\frac{3}{4} \pi \leq \arg z \leq-\frac{1}{4} \pi$, respectively. Then eq (2.6) shows that for large $|z|, U(a, z)$ is exponentially small in $\mathbf{M}_{0}$ and exponentially large in $\mathbf{M}_{1}$ and $\mathbf{M}_{3}$. Equation (2.7) shows that $U(a, z)$ is also exponentially large in $\mathbf{M}_{2}$, unless $a$ has one of the values $-\frac{1}{2},-\frac{3}{2},-\frac{5}{2}, \ldots$, in which case it is exponentially small. In fact in this event

$$
U\left(-n-\frac{1}{2},-z\right)=(-)^{n} U\left(-n-\frac{1}{2}, z\right) \quad(n=0,1,2, \ldots) .
$$

The solutions $U(a, \pm z), U(-a, \pm i z)$ clearly form a numerically satisfactory set in the complex plane, because in each sector $\mathbf{M}_{j}$ one of the solutions is exponentially small and at least two others exponentially large. Accordingly, we may expect that a knowledge of the asymptotic behavior of $U(a, z)$ for large $|a|$ in the sector $|\arg z| \leq \frac{3}{4} \pi$, or even the half-plane $|\arg z| \leq \frac{1}{2} \pi$, will enable us to determine the behavior of any solution of (2.1) over the whole z-plane by use of connection formulas.

Solutions when the variables are real. There are two forms of eq (2.1) of importance when the variables are real, namely

$$
\frac{d^{2} w}{d x^{2}}=\left(\frac{1}{4} x^{2}+a\right) w
$$

and

$$
\frac{d^{2} w}{d x^{2}}=\left(a-\frac{1}{4} x^{2}\right) w
$$

in which $a$ and $x$ are real. Equation (2.10) is obtained from (2.1) by setting $z=x e^{-\frac{1}{4} \pi i}$ and replacing $a$ by $i a$.

Real solutions of $(2.9)$ are $U(a, x)$ and $U(a,-x)$ but they do not form a satisfactory pair for all values of $a$; in particular they are linearly dependent when $a=-\frac{1}{2},-\frac{3}{2},-\frac{5}{2}, \ldots$. The fundamental solutions are taken to be $U(a, x)$ and $V(a, x)$, where

$$
V(a, x)=(1 / \pi) \Gamma\left(\frac{1}{2}+a\right)\{\sin \pi a \cdot U(a, x)+U(a,-x)\} .
$$

${ }^{2}$ Miller [4, p. 27], who is more concerned with asymptotic expansions fulfilling the uniqueness conditions of Watson [11] than the Poincaré condition, gives the range $|\arg z|<\frac{1}{2} \pi$. The validity, in Poincaré's sense, for the wider range is established in [14, p. 347]. 
For all values of $a, U(a, x)$ and $V(a, x)$ are linearly independent. When $a$ is negative it is often convenient to use in place of $V(a, x)$ its multiple $\bar{U}(a, x)$, given by

$$
\bar{U}(a, x)=\Gamma\left(\frac{1}{2}-a\right) V(a, x)=\tan \pi a \cdot U(a, x)+\sec \pi a \cdot U(a,-x) .
$$

When $a+\frac{1}{2}$ is a positive integer $\bar{U}(a, x)$ is infinite.

Wronskian relations for these solutions are

$$
\mathscr{W}\{U(a, x), V(a, x)\}=(2 / \pi)^{\frac{1}{2}}, \quad \mathscr{W}\{U(a, x), \bar{U}(a, x)\}=(2 / \pi)^{\frac{1}{2}} \Gamma\left(\frac{1}{2}-a\right) .
$$

The Hermite polynomials

$$
H_{n}(x)=(-)^{n} e^{x^{2}} \frac{d^{n}}{d x^{n}} e^{-x^{2}}
$$

are related to the function $U(a, x)$ by

$$
H_{n}(x)=2^{\frac{1}{2} n} e^{\frac{1}{2} x^{2}} U\left(-n-\frac{1}{2}, x \sqrt{ } 2\right) .
$$

For eq (2.10), the fundamental pair of real solutions is taken to be $W(a, x)$ and $W(a,-x)$, defined by

$$
\begin{gathered}
W(a, x)=(2 k)^{\frac{1}{2}} e^{\frac{1}{4} \pi a} \operatorname{Re}\left\{e^{i\left(\frac{1}{2} \phi_{2}+\frac{1}{8} \pi\right)} U\left(i a, x e^{-\frac{1}{4} \pi i}\right)\right\}, \\
W(a,-x)=(2 / k)^{\frac{1}{2} e^{\frac{1}{4} \pi a}} \operatorname{Im}\left\{e^{i\left(\frac{1}{2} \phi_{2}+\frac{1}{8} \pi\right)} U\left(i a, x e^{-\frac{1}{4} \pi i}\right)\right\} .
\end{gathered}
$$

where

$$
k=\left(1+e^{2 \pi a}\right)^{\frac{1}{2}}-e^{\pi a}, \quad 1 / k=\left(1+e^{2 \pi a}\right)^{\frac{1}{2}}+e^{\pi a} \quad \phi_{2}=\arg \Gamma\left(\frac{1}{2}+i a\right) .
$$

The value of arg $\Gamma\left(\frac{1}{2}+i a\right)$ here is not the principal one but is determined by the conditions that it is a continuous function of $a$ and equals $a \ln a-a+O\left(a^{-1}\right)$ as $a \rightarrow+\infty$.

The Wronskian relation is

$$
\mathscr{W}\{W(a, x), W(a,-x)\}=1 \text {. }
$$

Gamma function expansions. We shall need the asymptotic expansion of the function $\Gamma\left(\frac{1}{2}+z\right)$ and its reciprocal for large $|z|$. From Stirling's series [5, chapter 9], we have

$$
\ln \Gamma\left(\frac{1}{2}+z\right)=\frac{1}{2} \ln 2 \pi+z \ln z-z+Z(z)
$$

where

$$
Z(z) \sim \sum_{s=1}^{\infty} \frac{B_{2 s}\left(\frac{1}{2}\right)}{2 s(2 s-1)} \frac{1}{z^{2 s-1}} \equiv-\frac{1}{24} \frac{1}{z}+\frac{7}{2880} \frac{1}{z^{3}}-\frac{31}{40320} \frac{1}{z^{5}}+\ldots \quad(|\arg z| \leq \pi-\epsilon)
$$

uniformly with respect to $\arg z, B_{2 s}$ denoting the Bernoulli polynomial of degree $2 s$. Hence

$$
\Gamma\left(\frac{1}{2}+z\right) \sim(2 \pi)^{\frac{1}{2}} e^{-z} z^{z} \sum_{s=0}^{\infty} \frac{\gamma_{s}}{z^{s}} \quad(|\arg z| \leq \pi-\epsilon),
$$

where the constants are determined by the asymptotic identity

$$
\sum_{s=0}^{\infty} \frac{\gamma_{s}}{z^{s}} \sim \exp \{\boldsymbol{Z}(z)\}
$$

or equivalently,

$$
\sum_{s=1}^{\infty} \frac{s \gamma_{s}}{z^{s+1}} \sim\left\{\sum_{s=1}^{\infty} \frac{B_{2 s}\left(\frac{1}{2}\right)}{2 s} \frac{1}{z^{2 s}}\right\}\left\{\sum_{s=0}^{\infty} \frac{\gamma_{s}}{z^{s}}\right\} .
$$


Numerical calculation yields

$$
\gamma_{0}=1, \quad \gamma_{1}=-\frac{1}{24}, \quad \gamma_{2}=\frac{1}{1152}, \quad \gamma_{3}=\frac{1003}{414720}, \quad \gamma_{4}=-\frac{4027}{39813120} .
$$

Since $Z(z)$ is an odd function of $z$, it follows from (2.23) that

identically, and hence that

$$
\left\{\sum_{s=0}^{\infty} \frac{\gamma_{s}}{z^{s}}\right\}\left\{\sum_{s=0}^{\infty}(-)^{s} \frac{\gamma_{s}}{z^{s}}\right\} \sim 1
$$

$$
\frac{1}{\Gamma\left(\frac{1}{2}+z\right)} \sim \frac{e^{z} z^{-z}}{(2 \pi)^{\frac{1}{2}}} \sum_{s=0}^{\infty}(-)^{s} \frac{\gamma_{s}}{z^{s}} \quad(|\arg z| \leq \pi-\epsilon) .
$$

\section{Part 2. Expansions in Terms of Elementary Functions}

\section{Asymptotic Solutions of the Differential Equation}

In order to bring the differential eq (1.1) into the standard form required for the application of theorem $\mathrm{A}$ of [9], we take new variables $\xi=\xi(t)$ and $W$ (not the $W(a, x)$ of sec. 2), defined by

$$
\left(\frac{d \xi}{d t}\right)^{2}=t^{2}-1, \quad W=\left(t^{2}-1\right)^{\frac{1}{4}} w
$$

(cf. [7] sec. 2). Equation (1.1) then becomes

where

$$
\frac{d^{2} W}{d \xi^{2}}=\left\{\mu^{4}+\mathscr{F}(\xi)\right\} W
$$

$$
\mathscr{F}(\xi)=\left(t^{2}-1\right)^{-\frac{1}{4}} \frac{d^{2}}{d \xi^{2}}\left(t^{2}-1\right)^{\frac{1}{4}}=-\frac{3 t^{2}+2}{4\left(t^{2}-1\right)^{3}} .
$$

For convenience we prescribe that $\xi=0$ when $t=1$. From the first of eqs (3.1) we then obtain

and on expansion

$$
\xi=\int_{1}^{t}\left(t^{2}-1\right)^{\frac{1}{2}} d t=\frac{1}{2} t\left(t^{2}-1\right)^{\frac{1}{2}}-\frac{1}{2} \ln \left\{t+\left(t^{2}-1\right)^{\frac{1}{2}}\right\}
$$

$$
\xi=\frac{1}{2} t^{2}-\frac{1}{2} \ln 2 t-\frac{1}{4}+\frac{1}{4} \sum_{s=1}^{\infty} \frac{1.3 .5 \ldots(2 s-1)}{s(s+1) !\left(2 t^{2}\right)^{s}} \quad(|t|>1) .
$$

Temporarily we suppose that the branches of the many-valued functions occurring in (3.4) and (3.5) are positive when $t>1$ and determined by continuity elsewhere. The relation

$$
\xi\left(t e^{ \pm i \pi}\right)=\xi(t) \mp \frac{1}{2} i \pi
$$

follows immediately from (3.5) when $|t|>1$, and thence by analytic continuation throughout the $t$-plane cut along the join of $t=-1$ and $t=+1$.

The mapping of the $t$-plane, cut along the real axis from $-\infty$ to +1 , is illustrated in figure 1 . The center diagram corresponds to the upper half of the $t$-plane and the right-hand diagram to the lower half. They are of course conjugate to each other. Features of the mapping are that the upper side of the real $t$-axis is mapped on three straight lines BA $(\operatorname{Im} \xi=0, \operatorname{Re} \xi \geq 0)$, $\mathrm{AE}\left(\operatorname{Re} \xi=0,0 \geq \operatorname{Im} \xi \geq-\frac{1}{2} \pi\right), \quad \mathrm{ED}\left(\operatorname{Im} \xi=-\frac{1}{2} \pi, \quad \operatorname{Re} \xi \geq 0\right)$, and that the positive imaginary $t$-axis is mapped on the straight line OC $\left(\operatorname{Im} \xi=-\frac{1}{4} \pi, \quad \operatorname{Re} \xi \leq 0\right)$.

Next, we make the substitutions (cf. [9], sec. 1)

$$
u=|\mu|^{2}, \quad \theta=\arg \mu, \quad \xi=e^{-2 i \theta} x
$$




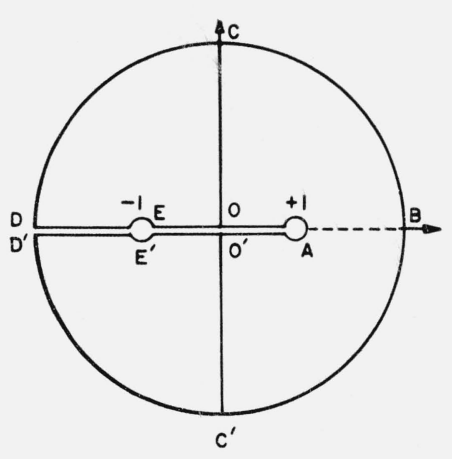

t-plane

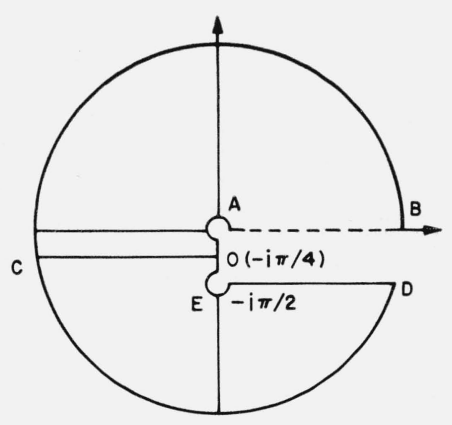

$\xi$-plane $(i)$

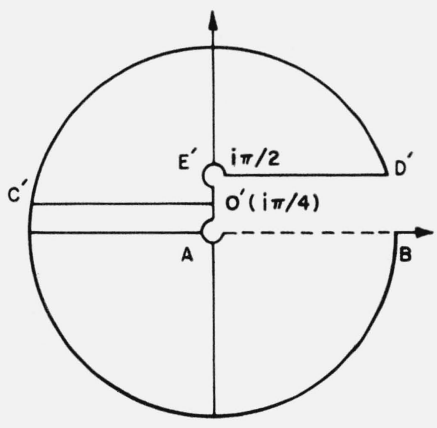

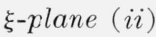

Figure 1. Mapping of the function $\xi(t)$.

(the $x$ here is not to be confused with the real variable of sec. 2). Then (3.2) becomes

$$
\frac{d^{2} W}{d x^{2}}=\left\{u^{2}+e^{-4 i \theta} \mathscr{F}\left(e^{-2 i \theta} x\right)\right\} W,
$$

which has the form of eq (2.1) of [9]. The relationship between $x$ and $t$ is given by

$$
x=x(t)=e^{2 i \theta} \xi(t)=e^{2 i \theta} \int_{1}^{t}\left(t^{2}-1\right)^{\frac{1}{2}} d t
$$

the $x$-map of the $t$-plane is obtained from the $\xi$-map by rotation through an angle $2 \theta$. The function $\mathscr{F}$ is expressed in terms of $t$ by (3.3). For large $|x|$ we deduce from (3.3) and (3.5) that

$$
e^{-4 i \theta} \mathscr{F}\left(e^{-2 i \theta} x\right) \sim-\frac{3}{4} e^{-4 i \theta} t^{-4} \sim-\frac{3}{16 x^{2}},
$$

unformly with respect to bounded $\arg x$ and bounded $\theta$.

In consequence of the last result we could, if we wished, take the $x$-domain $\mathbf{D}(\theta)$ of [9], section 2 to be the map of the $t$-plane cut along the real axis from $-\infty$ to +1 with the circles $|t \pm 1|\left\langle\delta^{\prime}\right.$ removed, $\delta^{\prime}$ being a fixed small positive number. The preliminary condition (2.2) of [9] would then be satisfied with $\sigma=1$. Greater regions of validity in the desired expansions will result however, if we arrange that the boundaries of $\mathbf{D}(\theta)$ are parallel to the positive imaginary $x$-axis where possible. Accordingly, we shall now investigate what we shall call the principal curves in the $t$-plane, namely the $t$-maps of the straight lines in the $\xi$-plane passing through $\mathrm{A}(\xi=0)$ and $\mathrm{E}\left(\xi=\mp \frac{1}{2} i \pi\right)$ and inclined at an angle $-2 \theta$ to the imaginary $\xi$-axis. In other words, the principal curves are the level curves of the function $e^{x(t)}$ which pass through $t= \pm 1$.

Near $t=1$ we find from (3.9) that

$$
x(t) \sim \frac{1}{3} 2^{\frac{3}{2}} e^{2 i \theta}(t-1)^{\frac{3}{2}} .
$$

Hence three principal curves emanate from $t=1$, and have as tangents the rays

$$
\arg (t-1)= \pm \frac{1}{3} \pi-\frac{4}{3} \theta, \quad \pi-\frac{4}{3} \theta
$$

Equation (3.6) shows that three principal curves also emanate from $t=-1$.

For large $|t|$ we have from (3.5)

$$
x(t) \sim \frac{1}{2} e^{2 i \theta} t^{2} .
$$


Hence the six principal curves are asymptotic to the four rays arg $t= \pm \frac{1}{4} \pi-\theta, \quad \pm \frac{3}{4} \pi-\theta$.

Figure 2 illustrates the principal curves when $0 \leq \theta<\pi$. Those through $\mathrm{A}$ are the continuous curves $\mathrm{AP}, \mathrm{AQ}, \mathrm{AS}$ when $0<\theta<\frac{1}{2} \pi$, and $\mathrm{AP}, \mathrm{AQ}, \mathrm{AR}$ when $\frac{1}{2} \pi<\theta<\pi$. When $\theta=0, \mathrm{AQ}$ degenerates into the curve $\mathrm{EQ}$ plus the join $\mathrm{AE}$, and when $\theta=\frac{1}{2} \pi, \mathrm{AS}, \mathrm{AR}$ degenerate into $\mathrm{AE}+\mathrm{ES}, \mathrm{AE}+\mathrm{ER}$, respectively. The principal curves through $\mathrm{E}$ are the images in the origin of those through $A$; this can be verified by using the continuation formula (3.6).

We can now define the branch of the many-valued function $\xi(t)$ which will be the most convenient for our purposes. When $0 \leq \theta<\pi$ we cut the $t$-plane along the principal curves $\mathrm{AQ}$ and ER and select the branch of $\xi(t)$ which is continuous in the cut plane and for which

$$
\xi(0)=\frac{1}{4} i \pi \quad(0<\theta<\pi), \quad \xi(0-0 i)=\frac{1}{4} i \pi \quad(\theta=0) .
$$

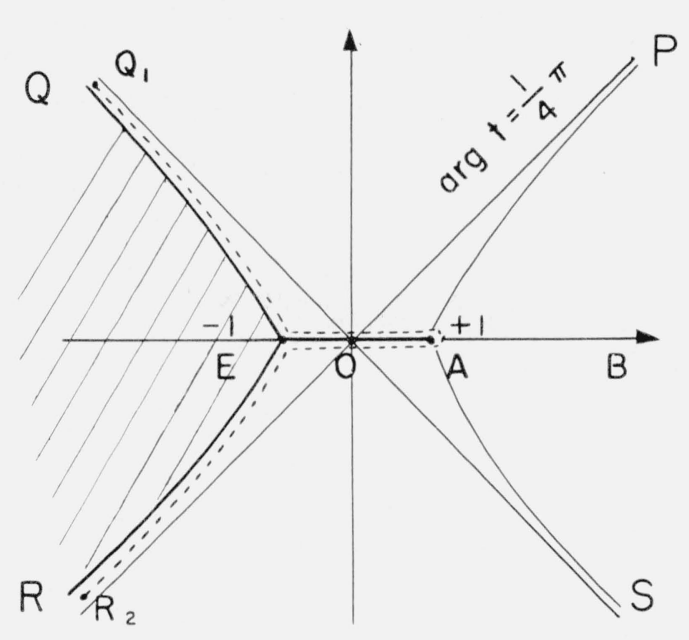

(a) $\theta=0$

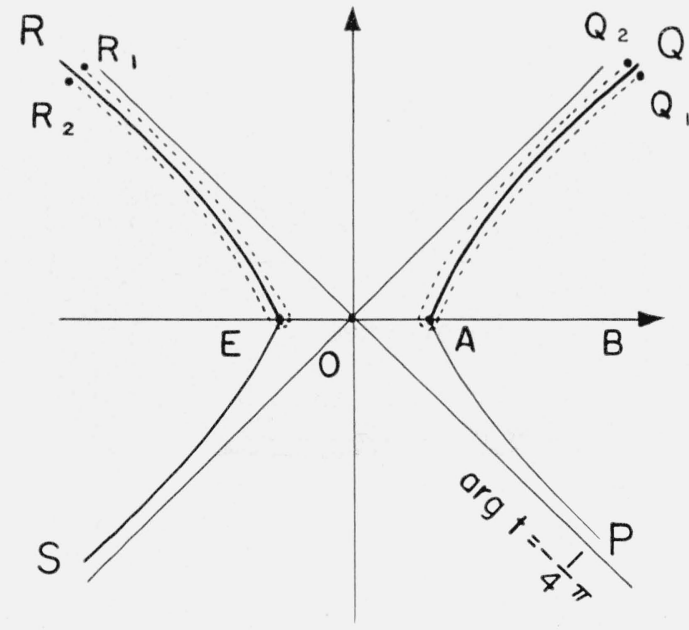

(c) $\theta=\frac{1}{2} \pi$

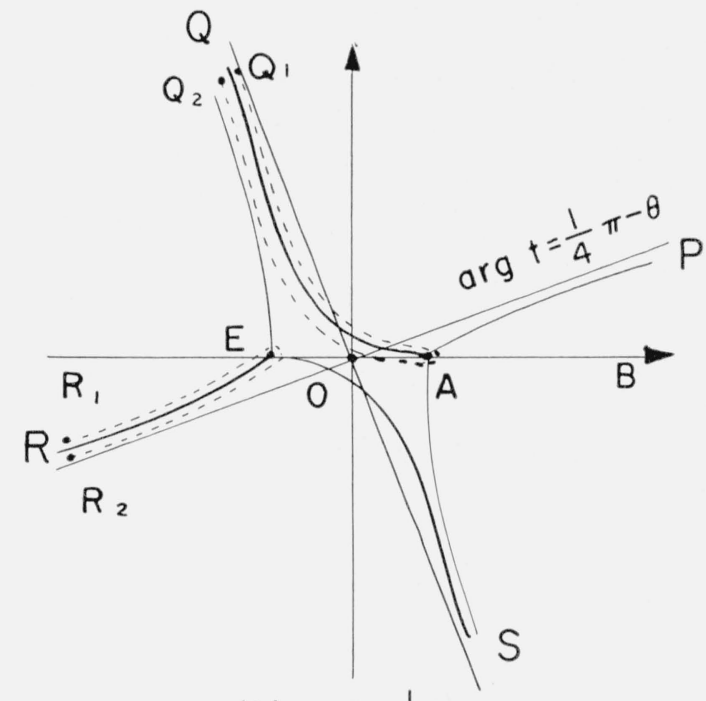

(b) $0<\theta<\frac{1}{2} \pi$

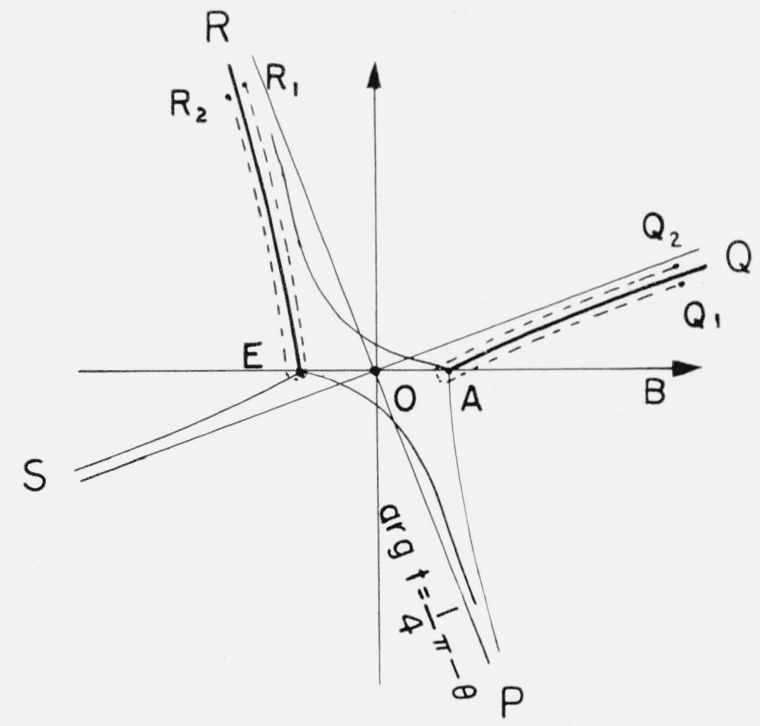

(d) $\frac{1}{2} \pi<\theta<\pi$

Figure 2. t-plane: principal curves and domains $\mathbf{S}(\theta)$. 
When $-\pi<\theta \leq 0$ the cuts are taken to be the conjugates of those for $-\theta$, and the branch is fixed by

$$
\xi(0)=-\frac{1}{4} i \pi \quad(-\pi<\theta<0), \quad \xi(0+0 i)=-\frac{1}{4} i \pi \quad(\theta=0) .
$$

It is evident from (3.6) that (3.13) and (3.14) are consistent when $\theta=0$. Henceforth we understand $\xi$ and $\xi(t)$ to denote this branch. For a given value of $\theta(-\pi<\theta<\pi), \quad \xi(t)$ is a singlevalued function of $t$ defined everywhere in the $t$-plane except when $\theta=0$ and $t$ lies in the shaded region to the left of $\mathrm{ER}+\mathrm{EQ}$ in figure $2(\mathrm{a})$. When $t>1, \xi(t)$ is positive provided that $|\theta|<\frac{3}{4} \pi$; this result is false if $\frac{3}{4} \pi<|\theta|<\pi$ for the cut AQ passes across the real axis as $\theta$ passes through $\frac{3}{4} \pi$.

Let $x \equiv x(t)$ now denote the branch $e^{2 i \theta} \xi(t)$. We then take the $x$-domain $\mathbf{D}(\theta)$ of [9], section 2 to be the map of the function $x(t)$ from which circles surrounding the points $\mathrm{A}$ and $\mathrm{E}$ and of radius $\frac{1}{2} \delta$ have been removed, $\delta$ being a fixed small positive number.

The map of $x(t)$ has two sheets when $\theta=0$ and three sheets when $0<|\theta|<\pi$. It is illustrated in figure 3 for $\theta=0$ and $0<\theta<\frac{1}{2} \pi$. The passage from one sheet to another takes place across the positive real axis and, when $0<\theta<\frac{1}{2} \pi$, the join AE. The heavy lines correspond to the cuts in the $t$-plane and are boundaries of $\mathbf{D}(\theta)$.

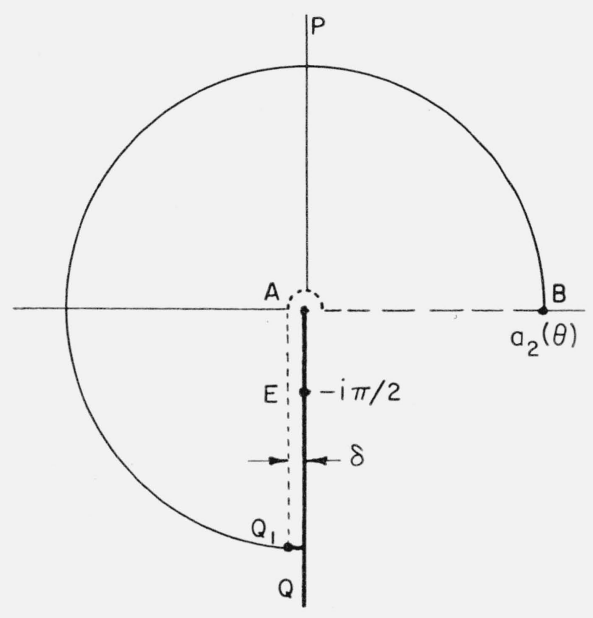

$\theta=0(i)$

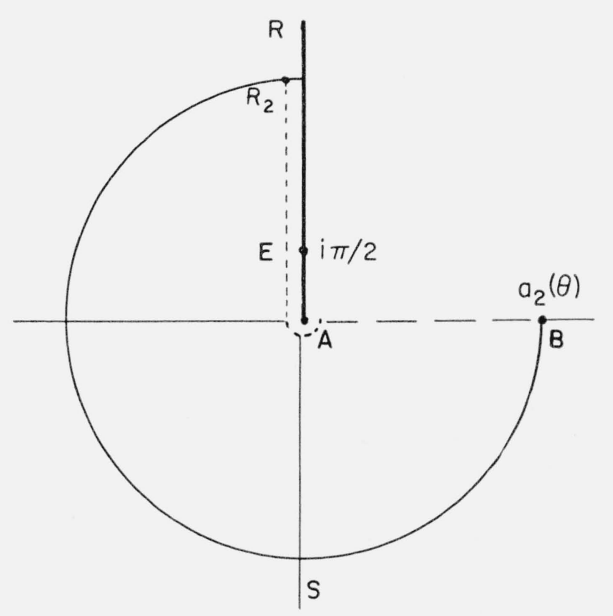

$\theta=0$ (ii)

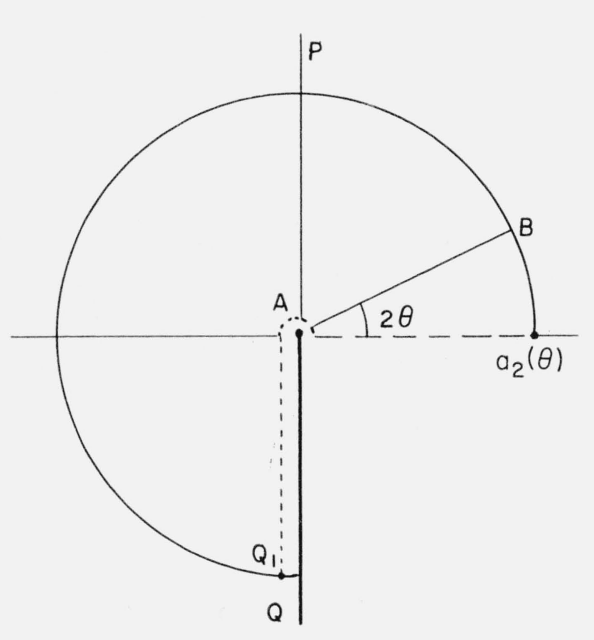

$0<\theta<\frac{1}{2} \pi(i)$

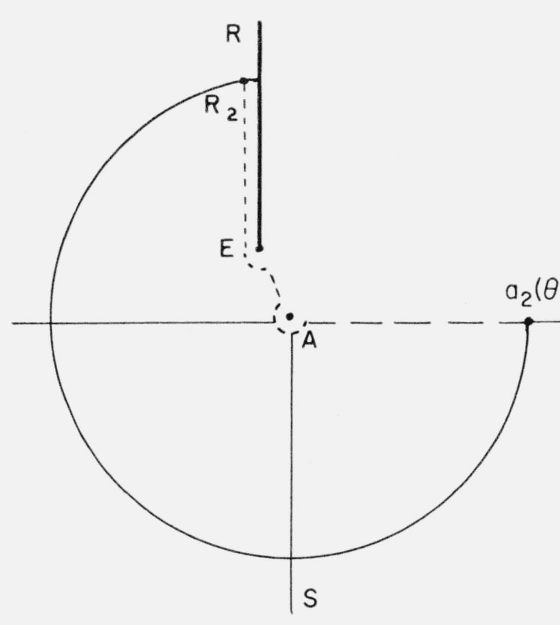

$0<\theta<\frac{1}{2} \pi$ (ii)

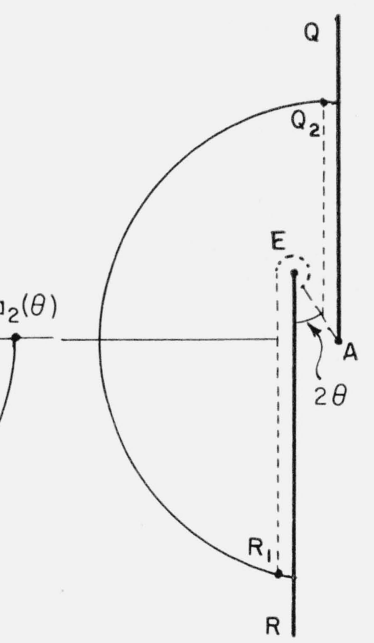

$0<\theta<\frac{1}{2} \pi($ iii $)$

Figure 3. $x$-plane: domains $\mathbf{D}(\theta)$ and $\mathbf{G}(\theta)$. 
The map of $x(t)$ is clearly a single unbounded domain. In order to ensure that $\mathbf{D}(\theta)$ is a single domain we have to impose the restriction $|\theta| \leq \pi-\epsilon$, omit sheet (iii) when $|\theta|<\epsilon$ and make $\delta=\delta(\epsilon)$ small enough. Otherwise, for example, the circle center E radius $\frac{1}{2} \delta$ would intersect the boundary $\mathrm{AQ}$.

Next, we define the $x$-domain $\mathbf{G}(\theta)$ required by [9], section 2 to be that part of the map of $x(t)$ which remains after removing all points at a distance less than $\delta$ from its boundaries. Sheet (iii) is again to be omitted when $|\theta|<\epsilon$. The boundaries of $\mathbf{G}(\theta)$ are the dotted lines and circular arcs of figure 3; their $t$-maps are the dotted curves of figure 2. Clearly when $|\theta| \leq \pi-\epsilon$, $\mathbf{G}(\theta)$ is a single domain if $\delta=\delta(\epsilon)$ is small enough. We readily show that conditions (i) and (ii) of [9], section 2 are fulfilled (cf. [9], sec. 3(i)).

Following [9], section 2 further, we take $a_{2}(\theta)$ to be the point at infinity on the positive real axis. We then find that $\mathbf{H}_{2}(\theta) \equiv \mathbf{G}(\theta)$. From theorem $\mathrm{A}$ it now follows that there exists a solution $W_{2}$ of eq (3.8) such that for large positive values of $u$

$$
W_{2} \sim e^{-u x} \sum_{s=0}^{\infty}(-)^{s} \frac{\mathscr{A}_{s}^{*}(\theta, x)}{u^{s}} \quad(x \in \mathbf{G}(\theta), \quad|\theta| \leq \pi-\epsilon),
$$

uniformly with respect to $x$ and $\theta$. The coefficients are given by the recurrence relation

$$
\mathscr{A}_{s+1}^{*}(\theta, x)=-\frac{1}{2} \frac{d}{d x} \mathscr{A}_{s}^{*}(\theta, x)+\frac{1}{2} \int e^{-4 i \theta} \mathscr{T}\left(e^{-2 i \theta} x\right) \mathscr{A}_{s}^{*}(\theta, x) d x,
$$

the path of integration lying in $\mathbf{D}(\theta)$.

If we now restore the variables $\mu$ and $\xi$ by means of eqs (3.7), and let

$$
\mathscr{A}_{s}(\xi) \equiv(-)^{s} e^{2 s i \theta} \mathscr{A}_{s}^{*}(\theta, x),
$$

the relations (3.15) and (3.16) become

$$
W_{2} \sim e^{-\mu^{2} \xi} \sum_{s=0}^{\infty} \frac{\mathscr{A}_{s}(\xi)}{\mu^{2 s}} \quad(t \epsilon \mathbf{S}(\arg \mu), \quad|\arg \mu| \leq \pi-\epsilon)
$$

as $|\mu| \rightarrow \infty$, where

$$
\mathscr{A}_{s+1}(\xi)=\frac{1}{2} \mathscr{A}_{s}^{\prime}(\xi)-\frac{1}{2} \int \mathscr{F}(\xi) \mathscr{A}_{s}(\xi) d \xi
$$

Here $\mathbf{S}(\arg \mu) \equiv \mathbf{S}(\theta)$ denotes the $t$-map of $\mathbf{G}(\theta)$; its boundaries are the dotted curves of figure 2 .

It is of some interest to determine the asymptotic behavior of the boundaries of $\mathbf{S}(\theta)$. Consider, for example, the dotted curve $A Q_{1}$. Its equation may be written

$$
\xi=(y-i \delta) e^{\left(\frac{3}{2} \pi-2 \theta\right) i},
$$

where the real parameter $y$ ranges from 0 to $+\infty$. Reversion of (3.5) yields

$$
t^{2}=2 \xi+\frac{1}{2} \ln 8 \xi+\frac{1}{2}+O\left(\xi^{-1} \ln \xi\right) \quad \text { as }|\xi| \rightarrow \infty .
$$

When $y$ is large, we may substitute (3.20) in (3.21) and thence obtain

$$
\arg t=\frac{3}{4} \pi-\theta+\frac{\cos 2 \theta}{8} \frac{\ln 8 y}{y}+\frac{\cos 2 \theta+\left(2 \theta-\frac{3}{2} \pi\right) \sin 2 \theta-4 \delta}{8 y}+O\left\{\left(\frac{\ln y}{y}\right)^{2}\right\} \text {. }
$$

The corresponding equation of the principal curve AQ is of course obtained on setting $\delta=0$ in this result. Clearly the principal curve $A Q$ and the dotted curve $A Q_{1}$ ultimately always lie on the same side of their common asymptote, except when $\theta=\frac{3}{4} \pi$, in which event $A Q$ is its own asymptote. 


\section{Identification of Solutions}

The substitutions

$$
a=-\frac{1}{2} \mu^{2}, \quad z=\mu t \sqrt{ } 2,
$$

transform eq (2.1) into (1.1). This result and the second of eqs (3.1) show that

$$
W=\left(t^{2}-1\right)^{\frac{1}{4}} U\left(-\frac{1}{2} \mu^{2}, \mu t \sqrt{ } 2\right)
$$

is a solution of (3.2). Equation (2.2) shows that when $\mu$ is fixed and $t \rightarrow \infty e^{-i \arg \mu}$ this solution becomes exponentially small. The same is true of the solution $W_{2}$ introduced in section 3 , provided that $|\mu|$ is sufficiently large and $|\arg \mu| \leq \pi-\epsilon$; this can be seen from (3.18) and (3.5). Since all solutions which are linearly independent of (4.2) are exponentially large in the same circumstances, we see immediately that the solution (4.2) is a multiple of $W_{2}$.

Hence we have

$$
U\left(-\frac{1}{2} \mu^{2}, \mu t \sqrt{ } 2\right) \sim g(\mu) \frac{e^{-\mu^{2} \xi}}{\left(t^{2}-1\right)^{\frac{1}{4}}} \sum_{s=0}^{\infty} \frac{\mathscr{A}_{s}(\xi)}{\mu^{2 s}} \quad(t \in \mathbf{S}(\arg \mu), \quad|\arg \mu| \leq \pi-\epsilon)
$$

as $|\mu| \rightarrow \infty$, uniformly with respect to $t$ and $\arg \mu$. The branch of $\left(t^{2}-1\right)^{\frac{1}{4}}$ here and elsewhere is understood to be continuous in $\mathbf{S}(\arg \mu)$ and to be asymptotic to the principal value of $\sqrt{ } t$ as $t \rightarrow \infty e^{-i \arg \mu}$. Similarly for the branch of $\left(t^{2}-1\right)^{\frac{1}{2}}$ used later. The function $g(\mu)$ is independent of $t$. Its asymptotic expansion for large $|\mu|$ can be found from the limiting form of the ratio of the two sides of the relation (4.3) as $t \rightarrow \infty e^{-i \arg \mu}$. Thus with the aid of (2.2) and (3.5) we find that

$$
\frac{1}{g(\mu)} \sim 2^{\frac{1}{4} \mu^{2}+\frac{1}{4}} e^{\frac{1}{4} \mu^{2}} \mu^{-\frac{1}{2} \mu^{2}+\frac{1}{2}} \sum_{s=0}^{\infty} \frac{g_{s}}{\mu^{2 s}} \quad(|\arg \mu| \leq \pi-\epsilon)
$$

as $|\mu| \rightarrow \infty$, uniformly with respect to $\arg \mu$. Here

$$
g_{s}=\lim \mathscr{A}_{s}(\xi) \quad \text { as } \xi \rightarrow \infty e^{-2 i \arg \mu} .
$$

That this limit exists and is independent of arg $\mu$ can be seen from the lemma of [9], section 5 . The $g_{s}$ are not determined however until the arbitrary constants in the recurrence relation (3.19) have been fixed, and we now consider how to do this in the most advantageous manner.

From (3.19), (3.3) and the first of (3.1), we obtain

$$
\mathscr{A}_{s+1}=\frac{1}{2} \frac{1}{\left(t^{2}-1\right)^{\frac{1}{2}}} \frac{d \mathscr{A}_{s}}{d t}+\frac{1}{8} \int \frac{3 t^{2}+2}{\left(t^{2}-1\right)^{\frac{5}{2}}} \mathscr{A}_{s} d t
$$

Taking $\mathscr{A}_{0}=1$, we find

$$
\mathscr{A}=\frac{t^{3}-6 t}{24\left(t^{2}-1\right)^{\frac{3}{2}}}+\text { constant. }
$$

A convenient value for this constant is zero, then $\mathscr{A}_{1}$ has the form of a polynomial in $t$ divided by $\left(t^{2}-1\right)^{\frac{3}{2}}$.

The eq (4.7) suggests the substitution

$$
\mathscr{A}_{s}=u_{s}(t) /\left(t^{2}-1\right)^{\frac{3}{2} s}
$$

in (4.6). This leads to the recurrence relation

where

$$
\left(t^{2}-1\right) u_{s}^{\prime}(t)-3 s t u_{s}(t)=r_{s-1}(t),
$$

$$
8 r_{s}(t)=\left(3 t^{2}+2\right) u_{s}(t)-12(s+1) t r_{s-1}(t)+4\left(t^{2}-1\right) r_{s-1}^{\prime}(t) .
$$


We can satisfy this relation by taking $u_{s}(t)$ to be a polynomial in $t$ of degree $3 s$ which is an odd or even function of $t$ according as $s$ is odd or even. All the coefficients are determined automatically if $s$ is odd, but there is a degree of freedom if $s$ is even, since the left of eq (4.9) vanishes when $u_{s}$ is a multiple of $\left(t^{2}-1\right)^{\frac{3}{2} s}$. We remove this by making the coefficient of the highest power of $t$ vanish.

Thus we prescribe that $u_{s}(t)$ is a polynomial in $t$ of degree $3 s$ ( $s$ odd), $3 s-2(s$ even, $s \geq 2)$. With $u_{0}(t)=1$ this condition determines the coefficients completely. Numerical calculation based on (4.9) and (4.10) yields

$$
\left.\begin{array}{l}
u_{0}(t)=1, \quad u_{1}(t)=\left(t^{3}-6 t\right) / 24, \quad u_{2}(t)=\left(-9 t^{4}+249 t^{2}+145\right) / 1152, \\
u_{3}(t)=\left(-4042 t^{9}+18189 t^{7}-28287 t^{5}-151995 t^{3}-259290 t\right) / 414720 .
\end{array}\right\}
$$

Hence

$$
g_{2 s}=0 \quad(s=1,2, \ldots), \quad g_{0}=1, \quad g_{1}=\frac{1}{24}, \quad g_{3}=-\frac{2021}{207360} .
$$

An independent method of calculating the asymptotic expansion of $g(\mu)$ for large $\mu$ will be given in section 5 .

Expansion for the derivative. Theorem A of [9] shows that the asymptotic series (4.3) may be differentiated term by term with respect to $t$. With the aid of the first of eqs (3.1) we deduce that

$$
U^{\prime}\left(-\frac{1}{2} \mu^{2}, \mu t \sqrt{ } 2\right) \sim-\frac{\mu}{\sqrt{ } 2} g(\mu)\left(t^{2}-1\right)^{\frac{1}{4}} e^{-\mu^{2} \xi} \sum_{s=0}^{\infty} \frac{\mathscr{B}_{s}(\xi)}{\mu^{2 s}} \quad(t \epsilon \mathbf{S}(\arg \mu), \quad|\arg \mu| \leq \pi-\epsilon)
$$

as $|\mu| \rightarrow \infty$, uniformly with respect to $t$ and $\arg \mu$. Here, in the notation of Miller,

$$
U^{\prime}(a, z) \equiv \partial U(a, z) / \partial z \text {. }
$$

The coefficients are given by

where

$$
\mathscr{B}_{s}(\xi)=v_{s}(t) /\left(t^{2}-1\right)^{\frac{3}{2} s}
$$

$$
v_{s}(t)=u_{s}(t)+\frac{1}{2} t u_{s-1}(t)-r_{s-2}(t)
$$

The first four $v_{s}(t)$ are

$$
\left.\begin{array}{l}
v_{0}(t)=1, \quad v_{1}(t)=\left(t^{3}+6 t\right) / 24, \quad v_{2}(t)=\left(15 t^{4}-327 t^{2}-143\right) / 1152, \\
v_{3}(t)=\left(-4042 t^{9}+18189 t^{7}-36387 t^{5}+238425 t^{3}+259290 t\right) / 414720 .
\end{array}\right\}
$$

\section{Use of Connection Formulas}

In this section we seek expansions which hold in the complement of the domain $\mathbf{S}(\arg \mu)$, Since $U(a, z)$ is an integral function of $a$ it will clearly suffice to obtain a set of expansions which, together with (4.3), cover the whole $t$-plane (except of course the neighborhoods of the points $t= \pm 1$ ) when $|\arg \mu| \leq \frac{1}{2} \pi$.

For convenience we introduce the notation $\mathbf{U}_{j} \equiv \mathbf{U}_{j}(\theta)(j=0,1,2,3,4)$ for the closed domains into which the $t$-plane is divided by the principal curves defined in section 3 . Figure 4 shows the enumeration of these regions when $0 \leq \theta<\pi$. When $-\pi<\theta \leq 0$ we define

$$
\mathbf{U}_{j}(\theta)=\mathbf{U}_{j}^{*}(-\theta) \quad(j=0,2,4), \quad \mathbf{U}_{1}(\theta)=\mathbf{U}_{3}^{*}(-\theta), \quad \mathbf{U}_{3}(\theta)=\mathbf{U}_{1}^{*}(-\theta),
$$

where the star denotes the conjugate region. Then the boundaries of each region $\mathbf{U}_{j}(\theta)$ vary continuously with $\theta$ in the interval $(-\pi, \pi)$. 


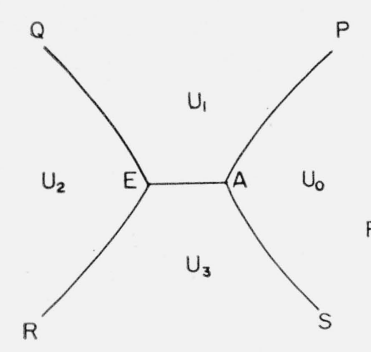

(a) $\theta=0$

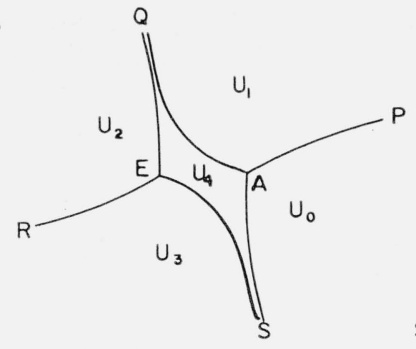

(b) $0<\theta<\frac{1}{2} \pi$

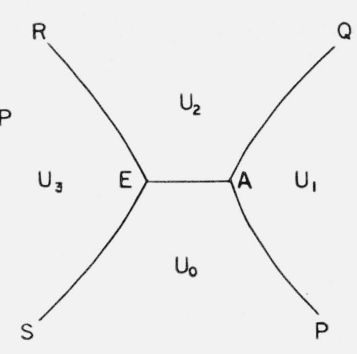

(c) $\theta=\frac{\pi}{2}$

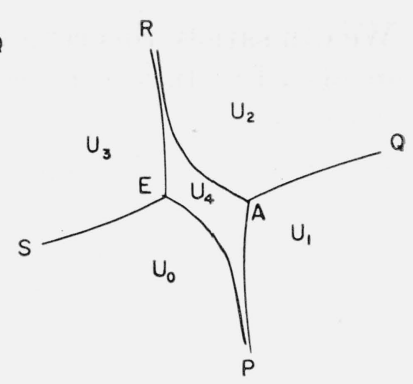

(d) $\frac{\pi}{2}<\theta<\pi$

Figure 4. t-plane: domains $\mathbf{U}_{j}(\theta)$.

The following relations, suggested by figure 4, are easily verified:

$$
\left.\begin{array}{c}
\mathbf{U}_{0}\left(\theta+\frac{1}{2} \pi\right)=\mathbf{U}_{3}(\theta), \quad \mathbf{U}_{1}\left(\theta+\frac{1}{2} \pi\right)=\mathbf{U}_{0}(\theta), \quad \mathbf{U}_{2}\left(\theta+\frac{1}{2} \pi\right)=\mathbf{U}_{1}(\theta), \\
\mathbf{U}_{3}\left(\theta+\frac{1}{2} \pi\right)=\mathbf{U}_{2}(\theta), \quad \mathbf{U}_{4}\left(\theta+\frac{1}{2} \pi\right)=\mathbf{U}_{4}(\theta),
\end{array}\right\}
$$

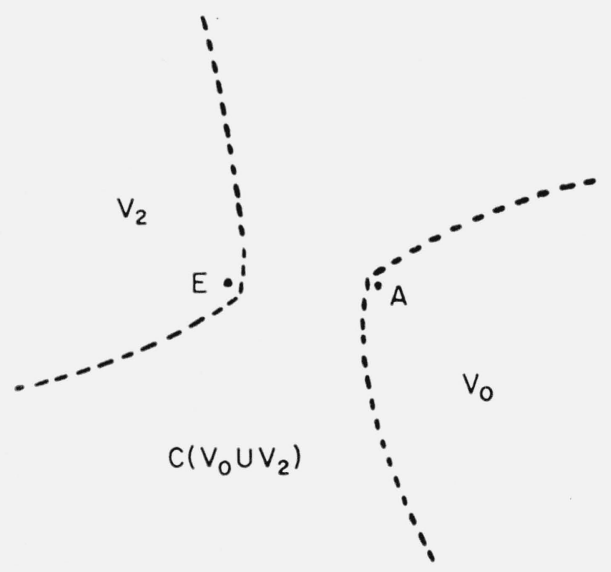

Figure 5. t-plane

$$
\left(0<\theta<\frac{1}{2} \pi\right)
$$

The region formed by adding to $\mathbf{U}_{j}(\theta)$ points whose $x$-maps (cf. fig. 3) are at a distance less than $\delta$ from the $x$-map of the boundary of $\mathbf{U}_{j}(\theta)$ we shall denote by $\mathbf{V}_{j}(\theta)$ or $\mathbf{V}_{j}$. Figure 5 illustrates for example $\mathbf{V}_{0}(\theta), \mathbf{V}_{2}(\theta)$, and $C\left\{\mathbf{V}_{0}(\theta) \cup \mathbf{V}_{2}(\theta)\right\}$, the complement of the union of $\mathbf{V}_{0}(\theta)$ and $\mathbf{V}_{2}(\theta)$, when $0<\theta<\frac{1}{2} \pi$. The boundaries are of the same character as the dotted curves of figure 2 .

The first connection formula we use is

$$
U\left(-\frac{1}{2} \mu^{2}, \mu t \sqrt{ } 2\right)=(2 \pi)^{-\frac{1}{2}} \Gamma\left(\frac{1}{2}+\frac{1}{2} \mu^{2}\right)\left\{e^{i \pi\left(-\frac{1}{4} \mu^{2}+\frac{1}{4}\right)} U\left(\frac{1}{2} \mu^{2},-i \mu t \sqrt{ } 2\right)+e^{i \pi\left(\frac{1}{4} \mu^{2}-\frac{1}{4}\right)} U\left(\frac{1}{2} \mu^{2}, i \mu t \sqrt{ } 2\right)\right\},
$$

obtained from (2.5). Replacing $\mu$ by $\mp i \mu$ in (4.3), we have

$$
U\left(\frac{1}{2} \mu^{2},-i \mu t \sqrt{ } 2\right) \sim g(-i \mu) \frac{e^{\mu^{2} \xi}}{\left(t^{2}-1\right)^{\frac{1}{4}}} \sum_{s=0}^{\infty}(-)^{s} \frac{\mathscr{A}_{s}(\xi)}{\mu^{2 s}} \quad\left(t \epsilon \mathbf{S}\left(\theta-\frac{1}{2} \pi\right), \quad-\frac{1}{2} \pi+\epsilon \leq \theta \leq \frac{3}{2} \pi-\epsilon\right),
$$


$U\left(\frac{1}{2} \mu^{2}, i \mu t \sqrt{ } 2\right) \sim g(i \mu) \frac{e^{\mu^{2} \xi}}{\left(t^{2}-1\right)^{\frac{1}{4}}} \sum_{s=0}^{\infty}(-)^{s} \frac{\mathscr{A}_{s}(\xi)}{\mu^{2 s}} \quad\left(t \epsilon \mathbf{S}\left(\theta+\frac{1}{2} \pi\right), \quad-\frac{3}{2} \pi+\epsilon \leq \theta \leq \frac{1}{2} \pi-\epsilon\right)$,

and from (4.4) and (4.12),

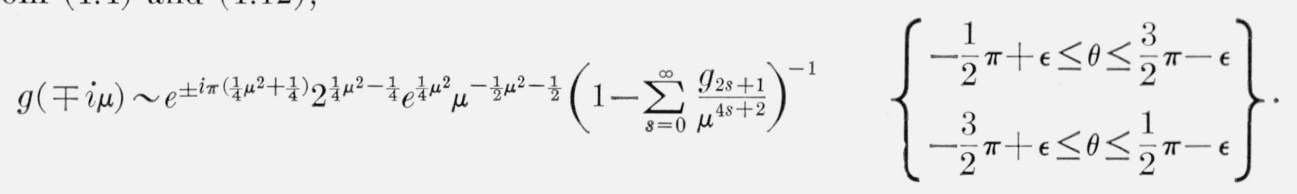

When $|\theta| \leq \frac{1}{2} \pi-\epsilon$, we can substitute (5.5) and (5.6) in (5.4). Care is required, however, because at any given point in the $t$-plane the branches of $\xi=\xi(t)$ and $\left(t^{2}-1\right)^{\frac{1}{4}}$ may not be the same in the two formulas.

When $0 \leq \theta \leq \frac{1}{2} \pi-\epsilon, \mathbf{S}\left(\theta-\frac{1}{2} \pi\right)$ is the complement of the region enclosed by "dotted curves" surrounding the principal curves AS and ER of figures $2(\mathrm{a})$ and $2(\mathrm{~b}) ; \mathbf{S}\left(\theta+\frac{1}{2} \pi\right)$ is the complement of the region enclosed by dotted curves surrounding AP and EQ. To the right of $\mathrm{AP}+\mathrm{AS}$, i.e., in $\mathbf{U}_{0}(\theta)$, the branches of $\xi(t)$ and $\left(t^{2}-1\right)^{\frac{1}{4}}$ are the same in (5.5) and (5.6). Substitution of these formulas in (5.4) leads to a nugatory result, however, as we would expect, since $e^{\mu^{2 \xi}}$ is exponentially large here whereas $U\left(-\frac{1}{2} \mu^{2}, \mu t \sqrt{ } 2\right)$ is exponentially small.

In the central region between $\mathrm{AP}+\mathrm{AS}$ and $\mathrm{EQ}+\mathrm{ER}$, i.e., in $C\left(\mathbf{U}_{0} \cup \mathbf{U}_{2}\right)$, the two branches of $\xi(t)$ have opposite signs. Let

$$
\xi=-i \eta \text { in }(5.5), \quad \xi=i \eta \text { in }(5.6) \text {. }
$$

Then $\eta$ is real and positive when $-1<t<1$; from (3.4) we find

$$
\eta=\int_{t}^{1}\left(1-t^{2}\right)^{\frac{1}{2}} d t=\frac{1}{2} \cos ^{-1} t-\frac{1}{2} t\left(1-t^{2}\right)^{\frac{1}{2}}
$$

The appropriate branches of $\left(t^{2}-1\right)^{\frac{1}{4}}$ and $\mathscr{A}_{s}(\xi)$ in $(5.5)$ are given by

$$
\left(t^{2}-1\right)^{\frac{1}{4}}=e^{\frac{1}{4} i \pi}\left(1-t^{2}\right)^{\frac{1}{4}}, \quad \mathscr{A}_{s}(\xi)=e^{-\frac{3}{2} i s \pi} u_{s}(t) /\left(1-t^{2}\right)^{\frac{3}{2} s}
$$

(cf. (4.8)), in which $\left(1-t^{2}\right)^{\frac{1}{4}}$ and $\left(1-t^{2}\right)^{\frac{3}{2} s}$ have their principal values when $-1<t<1$. For (5.6) we change the sign of $i$.

Carrying out the substitution, we obtain

$$
U\left(-\frac{1}{2} \mu^{2}, \mu t \sqrt{ } 2\right) \sim \frac{2 \tilde{g}(\mu)}{\left(1-t^{2}\right)^{\frac{1}{4}}}\left[\cos \left(\mu^{2} \eta-\frac{1}{4} \pi\right) \sum_{s=0}^{\infty} \frac{(-)^{s} u_{2 s}(t)}{\left(1-t^{2}\right)^{3 s} \mu^{4 s}}-\sin \left(\mu^{2} \eta-\frac{1}{4} \pi\right) \sum_{s=0}^{\infty} \frac{(-)^{s} u_{2 s+1}(t)}{\left(1-t^{2}\right)^{3 s+\frac{3}{2}} \mu^{4 s+2}}\right]
$$

as $|\mu| \rightarrow \infty$, uniformly with respect to $t \epsilon C\left(\mathbf{V}_{0} \cup \mathbf{V}_{2}\right)$ and $\theta$ in the interval $\left(-\frac{1}{2} \pi+\epsilon, \frac{1}{2} \pi-\epsilon\right)$. Here

$$
\begin{aligned}
\tilde{g}(\mu) & \sim(2 \pi)^{-\frac{1}{2}} \Gamma\left(\frac{1}{2}+\frac{1}{2} \mu^{2}\right) 2^{\frac{1}{4} \mu^{2}-\frac{1}{4}} e^{\frac{1}{4} \mu^{2}} \mu^{-\frac{1}{2} \mu^{2}-\frac{1}{2}}\left\{1-\sum_{s=0}^{\infty} \frac{g_{2 s+1}}{\mu^{4 s+2}}\right\}_{-1} \\
& \sim 2^{-\frac{1}{4} \mu^{2}-\frac{1}{4}} e^{-\frac{1}{4} \mu^{2}} \mu^{\frac{1}{2} \mu^{2}-\frac{1}{2}}\left\{\sum_{s=0}^{\infty} \frac{\gamma_{s}}{\left(\frac{1}{2} \mu^{2}\right)^{s}}\right\}\left\{1-\sum_{s=0}^{\infty} \frac{g_{2 s+1}}{\mu^{4 s+2}}\right\}_{-1} \quad\left(|\theta| \leq \frac{1}{2} \pi-\epsilon\right),
\end{aligned}
$$

on substituting for $\Gamma\left(\frac{1}{2}+\frac{1}{2} \mu^{2}\right)$ by means of $(2.22)$.

The expansion (5.11) is one of the required formulas. Before leaving it we shall show that $\widetilde{g}(\mu)=g(\mu)$, and in the process obtain a new derivation of the coefficients in the asymptotic expansion (4.4) for $1 / g(\mu)$.

When $\epsilon \leq \theta \leq \frac{1}{2} \pi-\epsilon$ the principal curve $\mathrm{AQ}$ is included in the region of validity of (5.11); this is easily proved by considering the $x$-map. On this curve $\mu^{2} \eta$ is positive. Let us set

$$
\eta=\eta_{m} \equiv\left(m+\frac{1}{4}\right) \frac{\pi}{\mu^{2}},
$$


where $m$ is a positive integer. Then (5.11) reduces to

$$
U\left(-\frac{1}{2} \mu^{2}, \mu t_{m} \sqrt{ } 2\right) \sim(-)^{m} \frac{2 \tilde{g}(\mu)}{\left(1-t_{m}^{2}\right)^{\frac{1}{4}}} \sum_{s=0}^{\infty} \frac{(-)^{s} u_{2 s}\left(t_{m}\right)}{\left(1-t_{m}^{2}\right)^{3 s} \mu^{4 s}}
$$

where $t_{m}$ is the value of $t$ corresponding to $\eta=\eta_{m}$.

Now keep $\mu$ fixed and let $m \rightarrow \infty$. Then $t_{m} \rightarrow \infty e^{i\left(\frac{3}{4} \pi-\theta\right)}$, and so from (2.7)

$$
U\left(-\frac{1}{2} \mu^{2}, \mu t_{m} \sqrt{ } 2\right) \sim e^{-\frac{1}{2} \mu^{2} t_{m}^{2}}\left(\mu t_{m} \sqrt{ } 2\right)^{\frac{1}{2} \mu^{2}-\frac{1}{2}}+\frac{(2 \pi)^{\frac{1}{2}} e^{i \pi\left(\frac{1}{2} \mu^{2}+\frac{1}{2}\right)}}{\Gamma\left(\frac{1}{2}-\frac{1}{2} \mu^{2}\right)} e^{\frac{1}{2} \mu^{2} t_{m}^{2}}\left(\mu t_{m} \sqrt{ } 2\right)^{-\frac{1}{2} \mu^{2}-\frac{1}{2}} .
$$

From (3.5) and the first of (5.8)

Hence

$$
\frac{1}{2} t_{m}^{2}-\frac{1}{2} \ln 2 t_{m}=-i \eta_{m}+\frac{1}{4}+O\left(\left|t_{m}\right|^{-2}\right) .
$$

$$
\begin{aligned}
& e^{-\frac{1}{2} \mu^{2} t_{m}^{2}}\left(\mu t_{m} \sqrt{ } 2\right)^{\frac{1}{2} \mu^{2}-\frac{1}{2}} \sim(-)^{m} e^{\frac{1}{4} \pi i-\frac{1}{4} \mu^{2}} 2^{-\frac{1}{4} \mu^{2-\frac{1}{4}}} \mu^{\frac{1}{2} \mu^{2}-\frac{1}{2}} t_{m}^{-\frac{1}{2}} \\
& e^{\frac{1}{2} \mu^{2} t_{m}^{2}}\left(\mu t_{m} \sqrt{ } 2\right)^{-\frac{1}{2} \mu^{2}-\frac{1}{2}} \sim(-)^{m} e^{-\frac{1}{4} \pi i+\frac{1}{4} \mu^{2}} 2^{\frac{1}{4} \mu^{2}-\frac{1}{4}} \mu^{-\frac{1}{2} \mu^{2}-\frac{1}{2}} t_{m}^{-\frac{1}{2}}
\end{aligned}
$$

and substituting these results in (5.15), we obtain

$$
(-)^{m} e^{\frac{1}{4} \pi i-\frac{1}{4} \mu^{2}} 2^{-\frac{1}{4} \mu^{2}-\frac{1}{4}} \mu^{\frac{1}{2} \mu^{2}-\frac{1}{2}}\left\{1+\frac{(2 \pi)^{\frac{1}{2}} e^{\frac{1}{2} i \pi \mu^{2}+\frac{1}{2} \mu^{2}} 2^{\frac{1}{2} \mu^{2}} \mu^{-\mu^{2}}}{\Gamma\left(\frac{1}{2}-\frac{1}{2} \mu^{2}\right)}\right\} t_{m}^{-\frac{1}{2}},
$$

as the limiting form of the left-hand side of (5.14).

On the right of (5.14) we have

$$
\left(1-t_{m}^{2}\right)^{\frac{1}{4}} \sim e^{-\frac{1}{4} \pi i} t_{m}^{\frac{1}{2}}, \quad u_{2 s}\left(t_{m}\right) /\left(1-t_{m}^{2}\right)^{3 s} \rightarrow 0 \quad(s \geq 1)
$$

(cf. (5.10), (4.5) and (4.12)). Comparing the two sides we see that

$$
\tilde{g}(\mu) \simeq 2^{-\frac{1}{4} \mu^{2}-\frac{1}{4}} e^{-\frac{1}{4} \mu^{2}} \mu^{\frac{1}{2} \mu^{2}-\frac{1}{2}}\left\{\frac{1}{2}+\frac{(2 \pi)^{\frac{1}{2}} e^{\frac{1}{2} i \pi \mu^{2}+\frac{1}{2} \mu^{2}} 2^{\frac{1}{2} \mu^{2}} \mu^{-\mu^{2}}}{2 \Gamma\left(\frac{1}{2}-\frac{1}{2} \mu^{2}\right)}\right\} \quad\left(\epsilon \leq \theta \leq \frac{1}{2} \pi-\epsilon\right),
$$

where we use the symbol $\simeq$ to mean that the two sides of this relation have the same asymptotic expansion for large $|\mu|$.

We now substitute $(2.27)$, with $z=-\frac{1}{2} \mu^{2}$, in (5.18) and obtain

$$
\tilde{g}(\mu) \sim 2^{-\frac{1}{4} \mu^{2}-\frac{1}{4}} e^{-\frac{1}{4} \mu^{2}} \mu^{\frac{1}{2} \mu^{2}-\frac{1}{2}}\left\{\frac{1}{2}+\frac{1}{2} \sum_{s=0}^{\infty} \frac{\gamma_{s}}{\left(\frac{1}{2} \mu^{2}\right)^{s}}\right\} .
$$

Although this result has been proved on the assumption that $\epsilon \leq \theta \leq \frac{1}{2} \pi-\epsilon$, comparison with (5.12) shows immediately that it holds in the range $|\theta| \leq \frac{1}{2} \pi-\epsilon$, and that

$$
\frac{1}{2}+\frac{1}{2} \sum_{s=0}^{\infty} \frac{\gamma_{s}}{\left(\frac{1}{2} \mu^{2}\right)^{s}} \sim\left\{\sum_{s=0}^{\infty} \frac{\gamma_{s}}{\left(\frac{1}{2} \mu^{2}\right)^{s}}\right\}\left\{1-\sum_{s=0}^{\infty} \frac{g_{2 s+1}}{\mu^{4 s+2}}\right\}^{-1}
$$

Identically. Thence using (2.26) and changing the sign of $\mu^{2}$, we find

$$
\left\{1+\sum_{s=0}^{\infty} \frac{g_{2 s+1}}{\mu^{4 s+2}}\right\}\left\{\frac{1}{2}+\frac{1}{2} \sum_{s=0}^{\infty} \frac{\gamma_{s}}{\left(\frac{1}{2} \mu^{2}\right)^{s}}\right\} \sim 1 .
$$

Multiplying this identity with (5.19), and comparing the result with (4.4) (remembering that $g_{2 s}=0$ if $s \geq 1$ ), we see that we can take

$$
\tilde{g}(\mu)=g(\mu)
$$


in the expansion (5.11), as stated earlier.

We can express the coefficients $g_{2 s+1}$ in terms of $\gamma_{s}$, by equating coefficients in (5.21): thus

$$
g_{1}=-\gamma_{1}=\frac{1}{24}, \quad g_{3}=-2 g_{1} \gamma_{2}-4 \gamma_{3}=-\frac{2021}{207360},
$$

on using (2.25). These values agree with those given in (4.12).

The expansion (5.11) is the first of our expansions for $U\left(-\frac{1}{2} \mu^{2}, \mu t \sqrt{ } 2\right)$ in regions complementary to $\mathbf{S}(\theta)$. The corresponding expansion for the derivative is

$$
\begin{aligned}
U^{\prime}\left(-\frac{1}{2} \mu^{2}, \mu t \sqrt{ } 2\right) \sim \mu \sqrt{2} g(\mu)\left(1-t^{2}\right)^{\frac{1}{4}}\left[\sin \left(\mu^{2} \eta-\frac{1}{4} \pi\right) \sum_{s=0}^{\infty}\right. & \frac{(-)^{s} v_{2 s}(t)}{\left(1-t^{2}\right)^{3 s} \mu^{4 s}} \\
& \left.\quad+\cos \left(\mu^{2} \eta-\frac{1}{4} \pi\right) \sum_{s=0}^{\infty} \frac{(-)^{s} v_{2 s+1}(t)}{\left(1-t^{2}\right)^{3 s+\frac{3}{2}} \mu^{4 s+2}}\right],
\end{aligned}
$$

valid under the same conditions as (5.11); the coefficients $v_{s}(t)$ are given oy (4.15) and (4.16).

A further expansion can be found by substituting (5.5) and (5.6) in (5.4) when $t$ lies in the region $\left(\mathbf{U}_{2}\right)$ to the left of $\mathrm{EQ}+\mathrm{ER}$. We shall not carry out this substitution however, because the expansion so obtained is contained in a more general result (5.28), which we shall now derive. As a preliminary we record the following relations in which the symbol $\simeq$ has the same meaning as in (5.18).

$$
\left.\begin{array}{rl}
g\left(\mu e^{i \pi}\right) & \simeq e^{i \pi\left(\frac{1}{2} \mu^{2}-\frac{1}{2}\right)} g(\mu) \quad(-\pi+\epsilon \leq \arg \mu \leq-\epsilon), \\
\frac{(2 \pi)^{\frac{1}{2}} e^{-i \pi\left(\frac{1}{4} \mu^{2}+\frac{1}{4}\right)}}{\Gamma\left(\frac{1}{2}-\frac{1}{2} \mu^{2}\right)} g\left(\mu e^{-\frac{1}{2} i \pi}\right) & \simeq 2 \cos \frac{1}{2} \pi \mu^{2} \cdot g(\mu) \quad\left(|\arg \mu| \leq \frac{1}{2} \pi-\epsilon\right) \\
& \simeq e^{-\frac{1}{2} i \pi \mu^{2}} g(\mu) \quad(\epsilon \leq \arg \mu \leq \pi-\epsilon) .
\end{array}\right\}
$$

We use the connection formula

$$
U\left(-\frac{1}{2} \mu^{2},-\mu t \sqrt{ } 2\right)=e^{2 \pi\left(-\frac{1}{2} \mu^{2}+\frac{1}{2}\right)} U\left(-\frac{1}{2} \mu^{2}, \mu t \sqrt{ } 2\right)+\frac{(2 \pi)^{\frac{1}{2}} e^{-i \pi\left(\frac{1}{4} \mu^{2}+\frac{1}{4}\right)}}{\Gamma\left(\frac{1}{2}-\frac{1}{2} \mu^{2}\right)} U\left(\frac{1}{2} \mu^{2},-i \mu t \sqrt{ } 2\right),
$$

obtained from (2.4). We may substitute the expansions (4.3) and (5.5) when

$$
-\frac{1}{2} \pi+\epsilon \leq \theta \leq \pi-\epsilon
$$

The branches of $\xi(t)$ are the same in both expansions when

$$
\left.\begin{array}{ll}
t \in C\left(\mathbf{V}_{2} \cup \mathbf{V}_{3} \cup \mathbf{V}_{4}\right) & \left(0 \leq \theta \leq \frac{1}{2} \pi\right), \\
t \in C\left(\mathbf{V}_{2} \cup \mathbf{V}_{3}\right) & \left(-\frac{1}{2} \pi+\epsilon \leq \theta \leq 0, \quad \frac{1}{2} \pi \leq \theta \leq \pi-\epsilon\right)
\end{array}\right\}
$$

Using (5.25) we obtain immediately

$$
\left.\begin{array}{cc}
U\left(-\frac{1}{2} \mu^{2},-\mu t \sqrt{ } 2\right) \sim \frac{g(\mu)}{\left(t^{2}-1\right)^{\frac{1}{4}}}\left[\left(\sin \frac{1}{2} \pi \mu^{2}+i \cos \frac{1}{2} \pi \mu^{2}\right) e^{-\mu^{2} \xi} \sum_{s=0}^{\infty} \frac{\mathscr{A}_{s}(\xi)}{\mu^{2 s}}\right. \\
\left.\quad+2 \cos \frac{1}{2} \pi \mu^{2} \cdot e^{\mu^{2 \xi}} \sum_{s=0}^{\infty}(-)^{s} \frac{\mathscr{A}_{s}(\xi)}{\mu^{2 s}}\right] \\
t \in C\left(\mathbf{V}_{2} \cup \mathbf{V}_{3}\right) & \left(-\frac{1}{2} \pi+\epsilon \leq \theta \leq 0\right), \\
t \in C\left(\mathbf{V}_{2} \cup \mathbf{V}_{3} \cup \mathbf{V}_{4}\right) & \left(0 \leq \theta \leq \frac{1}{2} \pi-\epsilon\right),
\end{array}\right\}
$$


and

$$
\left.\begin{array}{c}
U\left(-\frac{1}{2} \mu^{2},-\mu t \sqrt{2}\right) \sim e^{-\frac{1}{2} i \pi \mu^{2}} \frac{g(\mu)}{\left(t^{2}-1\right)^{\frac{1}{4}}}\left[i e^{-\mu^{2 \xi}} \sum_{s=0}^{\infty} \frac{\mathscr{A}_{s}(\xi)}{\mu^{2 s}}+e^{\mu^{2 \xi}} \sum_{s=0}^{\infty}(-)^{s} \frac{\mathscr{A}_{s}(\xi)}{\mu^{2 s}}\right] \\
t \in C\left(\mathbf{V}_{2} \cup \mathbf{V}_{3} \cup \mathbf{V}_{4}\right) \quad\left(\epsilon \leq \theta \leq \frac{1}{2} \pi\right), \\
t \in C\left(\mathbf{V}_{2} \cup \mathbf{V}_{3}\right) \quad\left(\frac{1}{2} \pi \leq \theta \leq \pi-\epsilon\right) .
\end{array}\right\}
$$

The last result may be expressed in a form more pertinent to the range $|\theta| \leq \frac{1}{2} \pi$ by replacing $\mu$ by $\mu e^{i \pi}$ and using (5.24). This gives

$$
\left.\begin{array}{c}
U\left(-\frac{1}{2} \mu^{2}, \mu t \sqrt{ } 2\right) \sim \frac{g(\mu)}{\left(t^{2}-1\right)^{\frac{1}{4}}}\left[e^{-\mu^{2} \xi} \sum_{s=0}^{\infty} \frac{\mathscr{A}_{s}(\xi)}{\mu^{2 s}}-i e^{\mu^{2 \xi}} \sum_{s=0}^{\infty}(-)^{s} \frac{\mathscr{A}_{s}(\xi)}{\mu^{2 s}}\right] \\
t \in C\left(\mathbf{V}_{0} \cup \mathbf{V}_{1}\right) \quad\left(-\frac{1}{2} \pi \leq \theta \leq-\epsilon\right) .
\end{array}\right\}
$$

Three further formulas, $\left(5.28^{*}\right),\left(5.29^{*}\right)$, and $\left(5.30^{*}\right)$, can be derived immediately from (5.28), (5.29), and (5.30), respectively, merely by changing the sign of $i$. They are valid with the conditions

$$
\begin{aligned}
& \left.\begin{array}{ll}
t \in C\left(\mathbf{V}_{1} \cup \mathbf{V}_{2} \cup \mathbf{V}_{4}\right) & \left(-\frac{1}{2} \pi+\epsilon \leq \theta \leq 0\right), \\
t \in C\left(\mathbf{V}_{1} \cup \mathbf{V}_{2}\right) & \left(0 \leq \theta \leq \frac{1}{2} \pi-\epsilon\right),
\end{array}\right\} \text { for }\left(5.28^{*}\right), \\
& \left.\begin{array}{ll}
t \in C\left(\mathbf{V}_{1} \cup \mathbf{V}_{2}\right) & \left(-\pi+\epsilon \leq \theta \leq-\frac{1}{2} \pi\right), \\
t \in C\left(\mathbf{V}_{1} \cup \mathbf{V}_{2} \cup \mathbf{V}_{4}\right) & \left(-\frac{1}{2} \pi \leq \theta \leq-\epsilon\right),
\end{array}\right\} \text { for }\left(5.29^{*}\right), \\
& t \in C\left(\mathbf{V}_{0} \cup \mathbf{V}_{3}\right) \quad\left(\epsilon \leq \theta \leq \frac{1}{2} \pi\right), \quad \text { for }\left(5.30^{*}\right) \text {. }
\end{aligned}
$$

\section{Summary of Expansions in Terms of Elementary Functions}

The principal expansion of this kind is (4.3). It is valid for large $|\mu|$ uniformly with respect to $\arg \mu \equiv \theta$ and $t$ when $-\pi+\epsilon \leq \theta \leq \pi-\epsilon$ and $t$ lies in the unbounded domain $\mathbf{S}(\theta)$, the boundaries of which are the dotted curves illustrated in figure 2. The function $\xi(t)$ is given by (3.4), that branch being chosen which is continuous in $\mathbf{S}(\theta)$ and takes the value (3.13) or (3.14) at the origin. The branch of $\left(t^{2}-1\right)^{\frac{1}{4}}$ in $(4.3)$ is continuous in $\mathbf{S}(\theta)$ and is asymptotic to the principal value of $\sqrt{ } t$ as $t \rightarrow \infty e^{-i \theta} \quad$ Both $\xi(t)$ and $\left(t^{2}-1\right)^{\frac{1}{4}}$ are positive when $t>1$ and $|\theta|<\frac{3}{4} \pi$. The coefficients $\mathscr{A}_{s}(\xi)$ are given by (4.8) and (4.11), the branch of $\left(t^{2}-1\right)^{\frac{3}{2} s}$ being defined similarly. The function $g(\mu)$ is calculable from either of the asymptotic expansions

$$
\begin{aligned}
& \frac{1}{g(\mu)} \sim 2^{\frac{1}{4} \mu^{2}+\frac{1}{4}} e^{\frac{1}{4} \mu^{2}} \mu^{-\frac{1}{2} \mu^{2}+\frac{1}{2}}\left\{1+\sum_{s=0}^{\infty} \frac{g_{2 s+1}}{\mu^{4 s+2}}\right\}, \\
& g(\mu) \sim 2^{-\frac{1}{4} \mu^{2}-\frac{1}{4}} e^{-\frac{1}{4} \mu^{2}} \mu^{\frac{1}{2} \mu^{2}-\frac{1}{2}}\left\{1+\frac{1}{2} \sum_{s=1}^{\infty} \frac{\gamma_{s}}{\left(\frac{1}{2} \mu^{2}\right)^{s}}\right\},
\end{aligned}
$$

both of which are uniformly valid with respect to $\arg \mu$ in the interval $(-\pi+\epsilon, \pi-\epsilon)$. Here $g_{1}, g_{3}$ are given by (4.12); $\gamma_{1}, \gamma_{2}, \gamma_{3}, \gamma_{4}$ by (2.25). 
Expansions valid in regions complementary to $\mathbf{S}(\theta)$ are (5.11), (5.28), (5.29), (5.30), $\left(5.28^{*}\right),\left(5.29^{*}\right)$, and $\left(5.30^{*}\right)$. When $0 \leq \theta \leq \frac{1}{2} \pi$ the expansions (5.28) and (5.29) between them cover the principal curve ER of figure 2 ; expansions $\left(5.28^{*}\right)$ and $\left(5.30^{*}\right)$ cover the principal curve $A Q$, except when $0 \leq \theta \leq \epsilon$ and $\operatorname{Re} t \geq-1-\delta^{\prime}$. The region of validity of (5.11) however includes that part of $\mathrm{AQ}$ to the right of $\operatorname{Re} t \geq-1+\delta^{\prime}$ when $0 \leq \theta \leq \epsilon$. Hence the expansions we have obtained cover the whole $t$-plane (except of course the neighborhoods of the points $t= \pm 1$ ) when $0 \leq \theta \leq \frac{1}{2} \pi$, and similarly also when $-\frac{1}{2} \pi \leq \theta \leq 0$.

The branches of the functions $\xi,\left(t^{2}-1\right)^{\frac{1}{4}}$ and $\mathscr{A}_{s}(\xi)$ in $(5.28),(5.29),(5.30)$, and the conjugate results are the same as in (4.3), and the function $g(\mu)$ again has the asymptotic expansions (6.1) and (6.2).

In $(5.11) \tilde{g}(\mu)$ is identical with $g(\mu)$, and $\eta=\eta(t)$ is given by (5.9). The branch of the latter function is continuous in the region of validity of (5.11) and positive when $-1<t<1$; the same is true of the branches of $\left(1-t^{2}\right)^{\frac{1}{4}}$ and $\left(1-t^{2}\right)^{\frac{3}{2}}$.

The expansions for the derivative $U^{\prime}\left(-\frac{1}{2} \mu^{2}, \mu t \sqrt{ } 2\right)$ corresponding to (4.3) and (5.11) are (4.13) and (5.23) respectively, the coefficients $\mathscr{B}_{s}(\xi)$ and $v_{s}(t)$ being given by (4.14) to (4.16). The derivative expansions corresponding to $(5.28)$ to $\left(5.30^{*}\right)$ have not been recorded, but they can be deduced immediately by use of the formal identities

$$
\frac{d}{d t} \frac{e^{ \pm \mu^{2} \xi}}{\left(t^{2}-1\right)^{\frac{1}{4}}} \sum_{s=0}^{\infty}(\mp)^{s} \frac{\mathscr{A}_{s}(\xi)}{\mu^{2 s}}= \pm \mu^{2}\left(t^{2}-1\right)^{\frac{1}{4}} e^{ \pm \mu^{2} \xi} \sum_{s=0}^{\infty}(\mp)^{s} \frac{\mathscr{B}_{s}(\xi)}{\mu^{2 s}}
$$

and are valid under the same conditions as the parent formulas.

Uniform asymptotic expansions of solutions of eq (1.1) for large $|\mu|$ and unbounded $t$ have previously been given by Watson [12], Schwid [10], and Darwin [1].

Watson applies the method of steepest descents to integral representations of

$$
D_{n}(z) \equiv U\left(-n-\frac{1}{2}, z\right)
$$

and obtains a set of asymptotic expansions for the function $D_{n}(2 \zeta \sqrt{ } n)$ when $|n|$ is large and $|\arg n| \leq \frac{1}{2} \pi-\epsilon$, which cover the whole $\zeta$-plane other than the neighborhoods of the points $\zeta= \pm 1$. For each expansion the $\zeta$-region of validity is fixed, unlike the regions obtained in the present paper which are permitted to depend on $\arg n$. For example, the region of validity of Watson's expansion analogous to our principal expansion (4.3) is

$$
\mathbf{U}_{0}\left(\frac{1}{4} \pi\right) \cup \mathbf{U}_{1}\left(\frac{1}{4} \pi\right) \quad\left(|\zeta-1| \geq \delta^{\prime}\right) .
$$

This is, as we would expect, the region common to $\mathbf{S}(\arg \mu)$ as $\arg \mu$ varies over the interval $\left(-\frac{1}{4} \pi+\epsilon, \frac{1}{4} \pi-\epsilon\right)$.

Schwid applies the methods of Langer for the asymptotic solution of differential equations and his results resemble those we have obtained. The main differences are that Schwid confines attention to the regions $\operatorname{Re} t \geq 0$ and $|\arg \mu|<\frac{3}{4} \pi$, gives only the leading terms in each expansion and identifies the asymptotic solutions by considering their behavior at $t=0$ rather than $t=\infty$.

Schwid's results apply to the even and odd solutions of the differential equation. An effect of carrying out the identification at $t=0$ is that the error term in the asymptotic representation of each of these solutions is obtained essentially in the form

$$
e^{-\mu^{2} \xi} O\left(|\mu|^{-2}\right)+e^{\mu^{2} \xi} O\left(|\mu|^{-2}\right)
$$

in the present notation. If we try to-deduce from these results the asymptotic form of the function $U\left(-\frac{1}{2} \mu^{2}, \mu t \sqrt{ } 2\right)$ by means of connection formulas, the error term will necessarily also be of the form (6.4). Accordingly, a nugatory result will be obtained in the region $\left(\mathbf{U}_{0}(\arg \mu)\right)$ in which $U\left(-\frac{1}{2} \mu^{2}, \mu t \sqrt{ } 2\right)$ is exponentially small for large $|\mu|$. Indeed, the proof given on page 
358 of Schwid's paper of the fact that the error term in the asymptotic expression for the Hermite polynomials is of the form $e^{-\mu^{2} \xi} O\left(|\mu|^{-2}\right)$ instead of (6.4) is false. The mistake is apparently due to overlooking the fact that the $O$ 's are functions of the independent variable as well as of the parameter $\mu$.

Darwin develops series solutions of the eq (2.10), and in [4] Miller applies the same methods to eq (2.1). The results are typified by the series

$$
U(a, z)=\frac{\left\{\Gamma\left(\frac{1}{2}-a\right)\right\}^{\frac{1}{2}}}{(2 \pi)^{\frac{1}{4}}} \exp \left\{-\theta-\frac{1}{2} \ln X+\sum_{s=1}^{\infty}(-)^{s} \frac{d_{3 s}}{X^{3 s}}\right\}
$$

for negative $a$ and real positive $z$, in which

$$
X=\sqrt{ }\left(z^{2}+4 a\right), \quad \theta=\frac{1}{2} \int_{2 \sqrt{ }(-a)}^{z} X d z,
$$

and $d_{3 s}$ is a polynomial in $z$ of degree $3 s$ if $s$ is odd, or $s$ if $s$ is even. Explicit expressions for $d_{3}, d_{6}, \ldots, d_{24}$ are given on page 68 of [4].

The series (6.5) is derived by purely formal methods from the differential equation without any investigation of its asymptotic nature. Applying the substitutions $a=-\frac{1}{2} \mu^{2}$ and $z=\mu t \sqrt{ } 2$ we find that $X=\mu\left(t^{2}-1\right)^{\frac{1}{2}} \sqrt{ } 2$ and $\theta=\mu^{2} \xi(t)$, in the notation of sections 3 and 4 . Next, replacing $\left\{\Gamma\left(\frac{1}{2}-a\right)\right\}^{\frac{1}{2}} \equiv\left\{\Gamma\left(\frac{1}{2}+\frac{1}{2} \mu^{2}\right)\right\}^{\frac{1}{2}}$ by its asymptotic expansion for large positive $\mu$ (cf. (2.22)) and expanding exp $\left\{\sum(-)^{s} d_{3 s} X^{-3 s}\right\}$ in descending powers of $X^{3}$, we find that the series (6.5) reduces to the form (4.3). Hence (6.5) must be a uniform asymptotic expansion for large $|a|$ of the same character as (4.3).

Darwin remarks that the explicit expressions for the coefficients $d_{3 s}$ are simpler in form and easier to calculate than the expressions for the coefficients in series of the form (4.3). This is indeed true, but we cannot however regard the form (6.5) as being entirely superior to $(4.3)$; it does not, for example, lead to convenient forms of expansion when we differentiate with respect to $z$.

\section{Part 3. Expansions in Terms of Airy Functions}

\section{Asymptotic Solutions of the Differential Equation}

In this section we seek to apply theorem B of [9] to the differential eq (1.1). The preliminary step is to take new variables $\zeta=\zeta(t)$ and $W$, defined by

$$
\zeta\left(\frac{d \zeta}{d t}\right)^{2}=t^{2}-1, \quad W=\left(\frac{d \zeta}{d t}\right)^{\frac{1}{2}} w=\left(\frac{t^{2}-1}{\zeta}\right)^{\frac{1}{4}} w
$$

(cf. [7], sec. 2). Equation (1.1) then becomes

where

$$
\frac{d^{2} W}{d \zeta^{2}}=\left\{\mu^{4} \zeta+F(\zeta)\right\} W
$$

$$
F(\zeta) \equiv\left(\frac{d t}{d \zeta}\right)^{\frac{1}{2}} \frac{d^{2}}{d \zeta^{2}}\left\{\left(\frac{d t}{d \zeta}\right)^{-\frac{1}{2}}\right\}=\frac{5}{16 \zeta^{2}}+\zeta \mathscr{F}(\xi)=\frac{5}{16 \zeta^{2}}-\frac{\left(3 t^{2}+2\right)}{4\left(t^{2}-1\right)^{3}} \zeta
$$

on reduction (cf. (3.3)).

The first of eqs (7.1) may be integrated to give

$$
\frac{2}{3} \zeta^{\frac{3}{2}}=\int_{1}^{t}\left(t^{2}-1\right)^{\frac{1}{2}} d t=\frac{1}{2} t\left(t^{2}-1\right)^{\frac{1}{2}}-\frac{1}{2} \ln \left\{t+\left(t^{2}-1\right)^{\frac{1}{2}}\right\},
$$

on specifying that $\xi=0$ when $t=1$. In terms of the variable $\xi$ of section 3 ,

$$
\frac{2}{3} \zeta^{\frac{3}{2}}=\xi \text {. }
$$


An essential difference between the functions $\xi(t)$ and $\zeta(t)$ is that the former has a branch point at $t=1$, whereas the latter is analytic at this point. Both functions have branch points at $t=-1$. The branch of $\zeta(t)$ which will be the most convenient for our purposes depends on $\theta \equiv \arg \mu$. We define it to be positive when $t>1$ and continuous in the $t$-plane cut along the principal curve ER of figure 2 when $0<\theta<\pi$, along the curve conjugate to ER when $-\pi<\theta<0$, or along $\mathrm{EQ}$ and $\mathrm{ER}$ when $\theta=0$. This determines $\zeta(t)$ uniquely everywhere except in the region $\left(\mathbf{U}_{2}(0)\right)$ to the left of $\mathrm{EQ}+\mathrm{ER}$ when $\theta=0$.

Following [9], section 1, we make the further substitutions

$$
u=|\mu|^{2}, \quad \theta=\arg \mu, \quad \zeta=e^{-\frac{4}{3} i \theta} z .
$$

Equation (7.2) then becomes

$$
\frac{d^{2} W}{d z^{2}}=\left\{u^{2} z+e^{-\frac{8}{3} i \theta} F\left(e^{-\frac{4}{3} i \theta} z\right)\right\} W
$$

The mapping of the $t$-plane on the z-plane is conveniently carried out by using $x(t)$ (defined in sec. 3) as an intermediate variable; in terms of $z$

$$
x=\frac{2}{3} z^{\frac{3}{2}}
$$

In our first application of theorem B to eq (7.7) we make a cut in the z-plane along the part of the level curve of the function $\exp \left(\frac{2}{3} z^{\frac{3}{2}}\right)$ which joins the point $\mathrm{E}$ of affix $-\left(\frac{3}{4} \pi\right)^{\frac{2}{3}} e^{\frac{4}{3} i \theta}$ and corresponding to $t=-1$, to the point at infinity on the negative real $z$-axis. Such a join can be made when $|\theta| \leq \frac{1}{2} \pi$ (cf. [7], fig. 2).

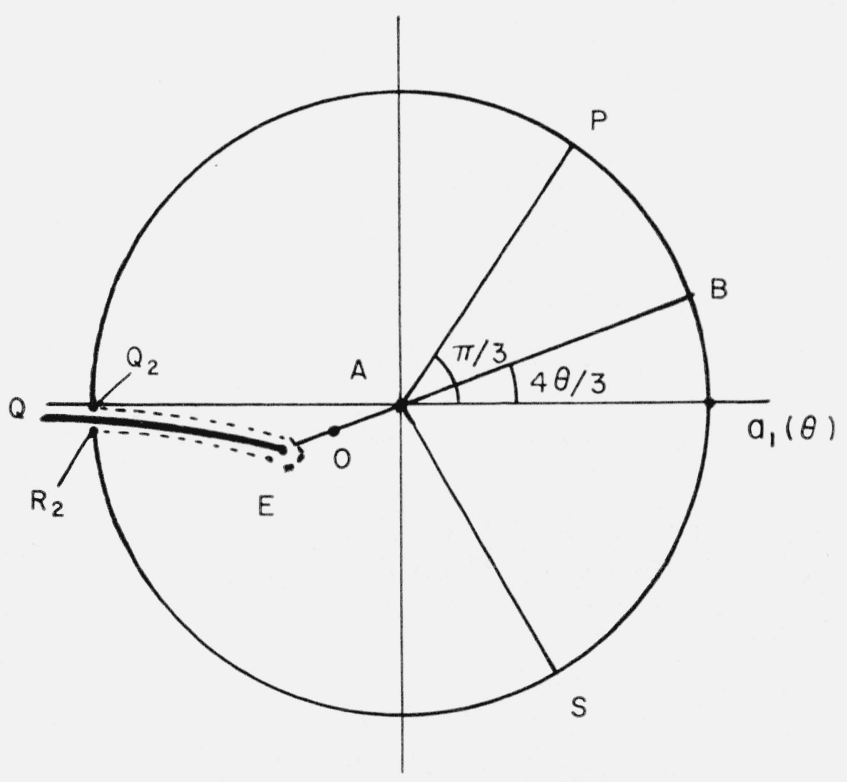

FIgure $6 . \quad z$-plane: domains

$\mathbf{D}(\theta)$ and $\mathbf{G}(\theta) \quad\left(|\theta| \leq \frac{1}{2} \pi-\epsilon\right)$.

Figure 6 illustrates the mapping, the lettering corresponding to figures 2 and 3 . The cut is the heavy curve EQ. The principal curves through $A$ are mapped on the rays $\arg z= \pm \frac{1}{3} \pi$ and $\pi$; the principal curves through $\mathrm{E}$ are mapped on the two sides of the cut EQ and the remaining part of the level curve of $\exp \left(\frac{2}{3} z^{\frac{3}{2}}\right)$ passing through E. Cut in this way, the z-plane is mapped on the complement of the domain $\mathbf{U}_{2}(\theta)$ (see fig. 4 ) in the $t$-plane.

When $|\theta| \leq \frac{1}{2} \pi-\epsilon$, we take the $z$-domain $\mathbf{D}(\theta)$ of [9], section 9 , to be the cut plane with the circle center $\mathrm{E}$ and radius $\frac{1}{2} \delta$ removed, $\delta$ being the fixed small positive number introduced in 
section 3. Then $F\left(e^{-\frac{4}{3} i \theta} z\right)$ is regular in $\mathbf{D}(\theta)$ (including $z=0$ ), and the condition (9.2) of [9] is satisfied with $\sigma=\frac{3}{2}$ because

$$
e^{-\frac{8}{3} i \theta} F\left(e^{-\frac{4}{3} \imath \theta} z\right) \sim-\frac{7}{64 z^{2}}
$$

as $|z| \rightarrow \infty$, uniformly with respect to bounded $\arg z$ and bounded $\theta$; this result follows from (7.3), (7.5), and (3.5).

We take the z-domain $\mathbf{G}(\theta)$ required by [9], section 9 , to be the complement of the map of the $t$-domain $\mathbf{V}_{2}(\theta)$, defined in section 5 . The boundaries of $\mathbf{G}(\theta)$ are the dotted curves surrounding the cut $\mathrm{EQ}$ in figure 6 . All the preliminary conditions of [9], section 9, are then satisfied if $\delta=\delta(\epsilon)$ is taken to be small enough.

Taking the point $a_{1}(\theta)$ of [9], section 9 to be at infinity on the positive real axis, we find $\mathbf{H}_{1}(\theta) \equiv \mathbf{G}(\theta)$. Hence from theorem $\mathrm{B}$, a solution $W_{1}$ of (7.7) exists, such that for large positive values of $u$

$$
W_{1} \sim \operatorname{Ai}\left(u^{\frac{2}{3}} z\right) \sum_{s=0}^{\infty} \frac{A_{s}(\theta, z)}{u^{2 s}}+\frac{\operatorname{Ai}^{\prime}\left(u^{\frac{2}{3}} z\right)}{u^{\frac{4}{3}}} \sum_{s=0}^{\infty} \frac{B_{s}(\theta, z)}{u^{2 s}} \quad\left(z \epsilon \mathbf{G}(\theta),|\theta| \leq \frac{1}{2} \pi-\epsilon\right)
$$

uniformly with respect to $z$ and $\theta$, the coefficients $A_{s}(\theta, z)$ and $B_{s}(\theta, z)$ being given by recurrence relations derived from eqs (9.12) and (9.13) of [9]. When the variables $\mu$ and $\zeta$ are restored, this result becomes

$$
W_{1} \sim \operatorname{Ai}\left(\mu^{\frac{4}{3}} \zeta\right) \sum_{s=0}^{\infty} \frac{A_{s}(\zeta)}{\mu^{4 s}}+\frac{\operatorname{Ai}^{\prime}\left(\mu^{\frac{4}{3}} \zeta\right)}{\mu^{\frac{8}{3}}} \sum_{s=0}^{\infty} \frac{B_{s}(\zeta)}{\mu^{4 s}}
$$

as $|\mu| \rightarrow \infty$, when

$$
t \epsilon C \mathbf{V}_{2}(\theta) \text { and }-\frac{1}{2} \pi+\epsilon \leq \theta \leq \frac{1}{2} \pi-\epsilon
$$

where $A_{0}(\zeta)=$ constant and

$$
\left.\begin{array}{l}
B_{s}(\zeta)=\frac{1}{2} \zeta^{-\frac{1}{2}} \int_{0}^{\zeta} \zeta^{-\frac{1}{2}}\left\{-A_{s}^{\prime \prime}(\zeta)+F(\zeta) A_{s}(\zeta)\right\} d \zeta \\
A_{s+1}(\zeta)=-\frac{1}{2} B_{s}^{\prime}(\zeta)+\frac{1}{2} \int^{\zeta} F(\zeta) B_{s}(\zeta) d \zeta
\end{array}\right\} \quad(s=0,1,2, \ldots)
$$

the paths of integration lying in the map of $C \mathbf{U}_{2}(\theta)$.

A second application of theorem $B$ can be made to the differential eq (7.7) by taking the cut in the $z$-plane for the function $F\left(e^{-\frac{4}{3} i \theta} z\right)$ to be the part of the level curve of exp $\left(\frac{2}{3} z^{\frac{3}{2}}\right)$ joining $\mathrm{E}$ to $\mathrm{S}$, the point at infinity on $\arg z=-\frac{1}{3} \pi$. This join is possible if $0 \leq \theta \leq \pi$; the corresponding $t$-domain is then $C \mathbf{U}_{3}(\theta)$.

With similar analysis, again taking $a_{1}(\theta)$ to be the point $z=+\infty$, we may show that a solution of eq (7.2) exists having the right-hand side of (7.10) as its asymptotic expansion for large $|\mu|$, uniformly valid with respect to $t$ and $\theta$ when

$$
t \in C \mathbf{V}_{3}(\theta) \text { and } \epsilon \leq \theta \leq \pi-\epsilon .
$$

The coefficients $A_{s}(\zeta)$ and $B_{s}(\zeta)$ are again given by (7.12), the paths of integration now lying in the map of $C \mathbf{U}_{3}(\theta)$.

By considering the limiting behavior as $\zeta \rightarrow \infty e^{-\frac{4}{3} i \theta}$, we see that when $\epsilon \leq \theta \leq \frac{1}{2} \pi-\epsilon$, the ratio of the new solution to $W_{1}$ is independent of $\zeta$ and has an asymptotic expansion for large $|\mu|$ identically equal to unity. Hence the expansion (7.10) for $W_{1}$ holds with the conditions (7.13). Similar analysis, or an appeal to symmetry, shows that this expansion also holds with the further conditions

$$
t \epsilon C \mathbf{V}_{1}(\theta) \text { and }-\pi+\epsilon \leq \theta \leq-\epsilon .
$$


We can combine the three $t$-regions of validity into a single region $\mathbf{T}(\theta)$, given by

$$
C \mathbf{V}_{1}, \quad C \mathbf{V}_{1} \cup C \mathbf{V}_{2}, \quad C \mathbf{V}_{2}, \quad C \mathbf{V}_{2} \cup C \mathbf{V}_{3}, \quad C \mathbf{V}_{3},
$$

according as $\theta$ lies in the respective intervals

$$
\left.\left(-\pi+\epsilon,-\frac{1}{2} \pi+\epsilon\right), \quad\left(-\frac{1}{2} \pi+\epsilon,-\epsilon\right), \quad(-\epsilon, \epsilon), \quad\left(\epsilon, \frac{1}{2} \pi-\epsilon\right), \quad\left(\frac{1}{2} \pi-\epsilon, \pi-\epsilon\right) .\right\}
$$

$\mathbf{T}(\theta)$ is illustrated in figure 7 for $0 \leq \theta \leq \pi-\epsilon ; \mathbf{T}(-\theta)$ is the conjugate of $\mathbf{T}(\theta)$. The dotted curves are of the same character as the dotted curves of figure 2 ; their $x$-maps parallel the $x$-maps of the principal curves through $\mathrm{E}$ at a distance $\delta$ away. $\mathbf{T}(\theta)$ is the unshaded domain bounded by the dotted curves.

Collecting together the results of this section, we have shown that there exists a solution $W_{1}$ of the differential eq (7.2) whose asymptotic expansion for large $|\mu|$ is given by (7.10) and is uniformly valid with respect to $t \epsilon \mathbf{T}(\theta)$ and $\theta$ in the interval $(-\pi+\epsilon, \pi-\epsilon)$. The functions $\zeta(t), F(\zeta), A_{s}(\zeta)$ and $B_{s}(\zeta)$ are given by (7.4), (7.3), and (7.12), and are regular when $t \epsilon \mathbf{T}(\theta)$.

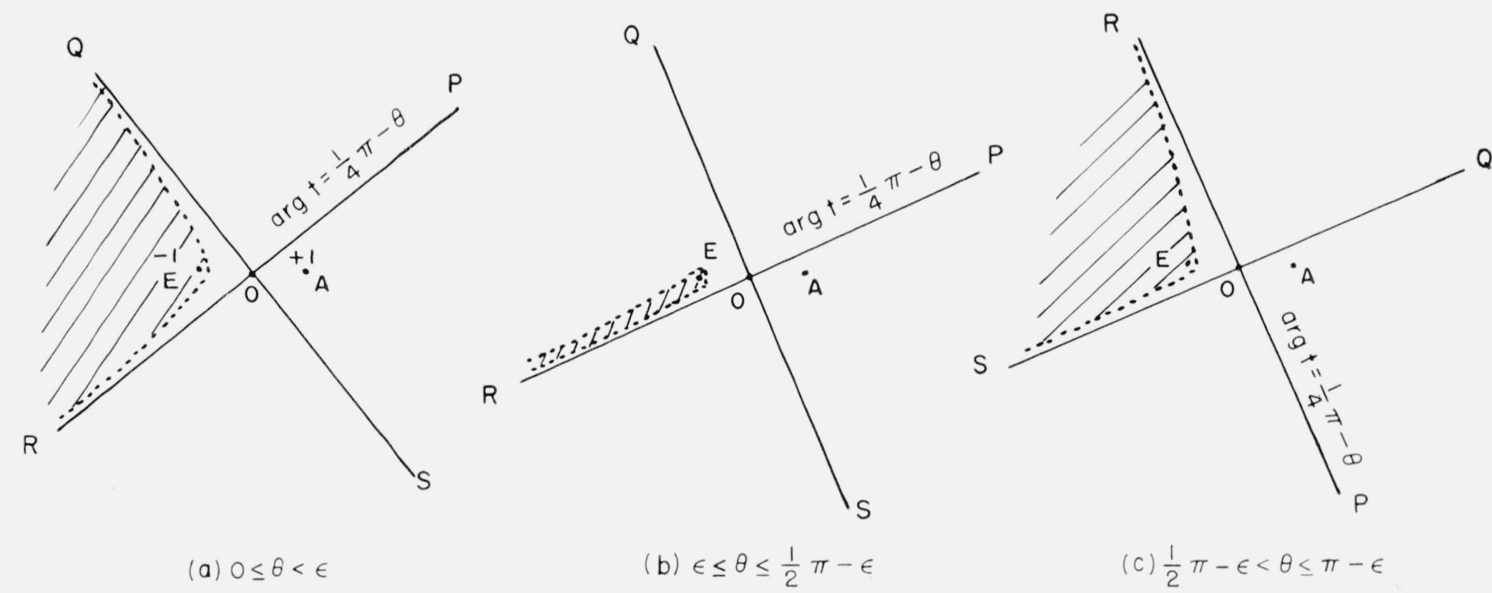

Figure 7. t-plane: domains $\mathbf{T}(\theta)$.

\section{Identification of Solutions}

From (4.1) and the second of eqs (7.1), we see that

$$
W=\left(\frac{t^{2}-1}{\zeta}\right) U\left(-\frac{1}{2} \mu^{2}, \mu t \sqrt{ } 2\right)
$$

satisfies the differential eq (7.2). When $\mu$ is fixed and $t \rightarrow \infty e^{-i \arg \mu}$ this solution becomes exponentially small. If $|\mu|$ is sufficiently large and $|\arg \mu| \leq \pi-\epsilon$, the solution $W_{1}$ of section 7 is also exponentially small in the same circumstances, for from $(7.4)$ we have $\zeta \sim\left(\frac{3}{4}\right)^{\frac{2}{3}} t^{\frac{4}{3}}$, and so $\mu^{\frac{4}{3}} \zeta \rightarrow+\infty$. Hence the two solutions are linearly dependent. Accordingly,

$$
U\left(-\frac{1}{2} \mu^{2}, \mu t \sqrt{ } 2\right) \sim h(\mu)\left(\frac{\zeta}{t^{2}-1}\right)^{\frac{1}{4}}\left[\mathrm{Ai}\left(\mu^{\frac{4}{3}} \zeta\right) \sum_{s=0}^{\infty} \frac{A_{s}(\zeta)}{\mu^{4 s}}+\frac{\mathrm{Ai}^{\prime}\left(\mu^{\frac{4}{3}} \zeta\right)}{\mu^{\frac{8}{3}}} \sum_{s=0}^{\infty} \frac{B_{s}(\zeta)}{\mu^{4 s}}\right]
$$

as $|\mu| \rightarrow \infty$, uniformly with respect to $t \epsilon \mathbf{T}(\arg \mu)$ and $\arg \mu$ in the interval $(-\pi+\epsilon, \pi-\epsilon)$.

This is the fundamental expansion in terms of Airy functions. In order to determine the function $h(\mu)$, we first fix the arbitrary constants of integration in the recurrence relations (7.12) by specifying the conditions

$$
A_{0}(\zeta)=1, \quad \lim _{|\zeta| \rightarrow \infty} A_{s}(\zeta)=0 \quad(s=1,2, \ldots) .
$$


We now examine the limiting form of the ratio of the two sides of the relation (8.2) as $t \rightarrow \infty e^{-i \operatorname{rrg} \mu}$. With the aid of (2.2), (3.5), (7.5), (8.3), and the asymptotic forms

$$
\operatorname{Ai}\left(\mu^{\frac{4}{3}} \zeta\right) \sim \frac{1}{2} \pi^{-\frac{1}{2}}\left(\mu^{\frac{4}{3}} \zeta\right)^{-\frac{1}{4}} e^{-\mu^{2} \xi}, \quad \operatorname{Ai}^{\prime}\left(\mu^{\frac{4}{3}} \zeta\right) \sim-\frac{1}{2} \pi^{-\frac{1}{2}}\left(\mu^{\frac{4}{3}} \zeta\right)^{\frac{1}{4}} e^{-\mu^{2} \xi}
$$

(cf. [8], appendix), we find that $1 / h(\mu)$ has the asymptotic expansion

$$
\frac{1}{h(\mu)} \sim \pi^{-\frac{1}{2}} 2^{\frac{1}{4} \mu^{2}-\frac{3}{4}} \mu^{-\frac{1}{2} \mu^{2}+\frac{1}{6}} e^{\frac{1}{4} \mu^{2}}\left(1-\sum_{s=0}^{\infty} \frac{h_{s}}{\mu^{4 s+2}}\right) \quad(|\arg \mu| \leq \pi-\epsilon)
$$

as $|\mu| \rightarrow \infty$, uniformly valid with respect to $\arg \mu$, where

$$
h_{s}=\lim _{|\zeta| \rightarrow \infty}\left\{\zeta^{\frac{1}{2}} B_{s}(\zeta)\right\} .
$$

An alternative form of expansion, not involving the quantities $h_{s}$, may be found as follows. Suppose that $\epsilon \leq \theta \leq \frac{1}{2} \pi-\epsilon$. The principal curve $A Q$ is then included in the domain $\mathbf{T}(\theta)$. On this curve $\mu^{\frac{4}{3}} \zeta$ is negative (cf. fig. 6). Let us set

$$
\zeta=\zeta_{m} \equiv \mu^{-\frac{4}{3}} a_{m}^{\prime},
$$

where $a_{m}^{\prime}$ is the $m$ th negative zero of the function $\mathrm{Ai}^{\prime}$. Then (8.2) becomes

$$
U\left(-\frac{1}{2} \mu^{2}, \mu t_{m} \sqrt{ } 2\right) \sim h(\mu)\left(\frac{\zeta_{m}}{t_{m}^{2}-1}\right)^{\frac{1}{4}} \mathrm{Ai}\left(a_{m}^{\prime}\right) \sum_{s=0}^{\infty} \frac{A_{s}\left(\zeta_{m}\right)}{\mu^{4 s}}
$$

in which $t_{m}$ denotes the value of $t$ corresponding to $\zeta=\zeta_{m}$. Now keep $\mu$ fixed and let $m \rightarrow \infty$, so that $t \rightarrow \infty e^{i\left(\frac{3}{4} \pi-\theta\right)}$. Then from [8], appendix, we have

$$
a_{m}^{\prime}=-\left\{\frac{3}{8} \pi(4 m-3)\right\}^{\frac{2}{3}}\left\{1+O\left(m^{-2}\right)\right\}, \quad \operatorname{Ai}\left(a_{m}^{\prime}\right)=(-)^{m-1} \pi^{-\frac{1}{2}}\left(-a_{m}^{\prime}\right)^{-\frac{1}{4}}\left\{1+O\left(m^{-2}\right)\right\},
$$

and from (7.5) and (3.5)

$$
t_{m}^{2}-\ln 2 t_{m}=\frac{4}{3} \zeta_{m}^{\frac{3}{2}}+\frac{1}{2}+O\left(\left|t_{m}\right|^{-2}\right) .
$$

Substituting in (2.7) by means of these equations, we find that the limiting form of the lefthand side of (8.7) is minus the expression (5.17), with $t_{n}$ defined of course as in the present section. On the other hand, the limiting form of the right of (8.7) is

$$
h(\mu)\left(\frac{\mu^{-\frac{4}{3}} a_{m}^{\prime}}{t_{m}^{2}-1}\right)^{\frac{1}{4}}(-)^{m-1} \pi^{-\frac{1}{2}}\left(-a_{m}^{\prime}\right)^{-\frac{1}{4}} \sim(-)^{m-1} \pi^{-\frac{1}{2}} e^{\frac{i \pi}{4}} \mu^{-\frac{1}{3}} h(\mu) t_{m}^{-\frac{1}{2}}
$$

(cf. (8.3) and (8.8)). Comparing the two sides we find that $\frac{1}{2} \pi^{-\frac{1}{2}} \mu^{-\frac{1}{3}} h(\mu)$ has the same asymptotic expansion for large $|\mu|$ as the right-hand side of the relation (5.18). Hence we obtain

$$
h(\mu) \simeq 2 \pi^{\frac{1}{2}} \mu^{\frac{1}{3}} \tilde{g}(\mu) \simeq 2 \pi^{\frac{1}{2}} \mu^{\frac{1}{3}} g(\mu),
$$

where $\tilde{g}(\mu), g(\mu)$ are defined in sections 4 and $5 ; g(\mu)$ has the asymptotic expansions (6.1) and (6.2) for large $|\mu|$. Although (8.10) has been proved on the assumption that $\epsilon \leq \arg \mu \leq \frac{1}{2} \pi-\epsilon$, (8.4) shows that $1 / h(\mu)$, and hence also $h(\mu)$, possesses a single asymptotic expansion over the range $|\arg \mu| \leq \pi-\epsilon$. Therefore (8.10) must also hold over this wider range of arg $\mu$.

Substituting (8.10) in (8.2), we obtain the final form of the fundamental expansion, given by

$$
U\left(-\frac{1}{2} \mu^{2}, \mu t \sqrt{ } 2\right) \sim 2 \pi^{\frac{1}{2}} \mu^{\frac{1}{3}} g(\mu)\left(\frac{\zeta}{t^{2}-1}\right)^{\frac{1}{4}}\left[\operatorname{Ai}\left(\mu^{\frac{4}{3}} \zeta\right) \sum_{s=0}^{\infty} \frac{A_{s}(\zeta)}{\mu^{4 s}}+\frac{\operatorname{Ai}^{\prime}\left(\mu^{\frac{4}{3}} \zeta\right)}{\mu^{\frac{8}{3}}} \sum_{s=0}^{\infty} \frac{B_{s}(\zeta)}{\mu^{4 s}}\right]
$$

as $|\mu| \rightarrow \infty$, uniformly with respect to $t \epsilon \mathbf{T}(\arg \mu), \arg \mu$ in the interval $(-\pi+\epsilon, \pi-\epsilon)$. 
The coefficients $A_{s}(\zeta)$ and $B_{s}(\zeta)$ appearing in (8.11) are determined by the recurrence relations (7.12) and the conditions (8.3). Explicit expressions can be found by the method used in [8], section 6 for the determination of the coefficients in the uniform expansions of Bessel functions of large order. We take $\zeta$ to be fixed and positive, and let $\mu \rightarrow+\infty$. The Airy functions appearing in (8.11) can then be expanded in their asymptotic series for large positive arguments and the result compared with (4.3). Thus we derive the expressions

$$
A_{s}(\zeta)=\sum_{m=0}^{2 s} b_{m} \zeta^{-\frac{3}{2} m} \mathscr{A}_{2 s-m}(\xi), \quad \zeta^{\frac{1}{2}} B_{s}(\zeta)=-\sum_{m=0}^{2 s+1} a_{m} \zeta^{-\frac{3}{2} m} \mathscr{A}_{2 s-m+1}(\xi)
$$

(cf. [8], (6.6)), where $\mathscr{A}_{m}(\xi)$ is defined in section $4, a_{0}=1$ and

$$
a_{m}=\frac{(2 m+1)(2 m+3) \ldots(6 m-1)}{m !(144)^{m}}, \quad b_{m}=-\frac{6 m+1}{6 m-1} a_{m} .
$$

Analytic continuation shows that eqs (8.12) hold for all values of $\zeta$.

We note from $(8.10),(8.4)$, and (6.1) that

$$
h_{s} \equiv \lim _{|\zeta| \rightarrow \infty}\left\{\zeta^{\frac{1}{2}} B_{s}(\zeta)\right\}=-g_{2 s+1} \quad(s=0,1, \ldots),
$$

where $g_{s}$ is given by (4.12) and (5.21).

Expansion for the derivative. The expansion (8.11) may be differentiated term by term with respect to $t$; this is a consequence of theorem $B$. With the aid of the first of eqs (7.1), we find that

$$
U^{\prime}\left(-\frac{1}{2} \mu^{2}, \mu t \sqrt{2}\right) \sim \frac{(2 \pi)^{\frac{1}{2}} \mu^{\frac{2}{3}} g(\mu)}{\phi(\zeta)}\left[\frac{\operatorname{Ai}\left(\mu^{\frac{4}{3}} \zeta\right)}{\mu^{\frac{4}{3}}} \sum_{s=0}^{\infty} \frac{C_{s}(\zeta)}{\mu^{4 s}}+\operatorname{Ai}^{\prime}\left(\mu^{\frac{4}{3}} \zeta\right) \sum_{s=0}^{\infty} \frac{D_{s}(\zeta)}{\mu^{4 s}}\right]
$$

as $|\mu| \rightarrow \infty$, uniformly with respect to $t \epsilon \mathbf{T}(\arg \mu)$ and $\arg \mu$ in the interval $(-\pi+\epsilon, \pi-\epsilon)$.

Here we have used the notation

$$
\phi(\zeta) \equiv\left(\frac{\zeta}{t^{2}-1}\right)^{\frac{1}{4}}=\left(\frac{d t}{d \zeta}\right)^{\frac{1}{2}} .
$$

The coefficients $C_{s}(\zeta)$ and $D_{s}(\zeta)$ are expressed in terms of $A_{s}(\zeta)$ and $B_{s}(\zeta)$ by the equations

$$
C_{s}(\zeta)=\chi(\zeta) A_{s}(\zeta)+A_{s}^{\prime}(\zeta)+\zeta B_{s}(\zeta), \quad D_{s}(\zeta)=A_{s}(\zeta)+\chi(\zeta) B_{s-1}(\zeta)+B_{s-1}^{\prime}(\zeta)
$$

(cf. [8], (6.10)), where

$$
\chi(\zeta) \equiv \frac{\phi^{\prime}(\zeta)}{\phi(\zeta)}=\frac{1}{4 \zeta}-\frac{t}{2} \frac{\zeta^{\frac{1}{2}}}{\left(t^{2}-1\right)^{\frac{3}{2}}}=\frac{1-2 t\{\phi(\zeta)\}^{6}}{4 \zeta}
$$

Explicitly,

$$
\zeta^{-\frac{1}{2}} C_{s}(\zeta)=-\sum_{m=0}^{2 s+1} b_{m} \zeta^{-\frac{3}{2} m} \mathscr{B}_{2 s-m+1}(\xi), \quad D_{s}(\zeta)=\sum_{m=0}^{2 s} a_{m} \zeta^{-\frac{3}{2} m} \mathscr{B}_{2 s-m}(\xi),
$$

(cf. [8], (6.12)), where $\mathscr{B}_{m}(\xi)$ is given by (4.14) to (4.16), and $a_{m}, b_{m}$ by (8.13).

\section{Use of Connection Formulas}

In this section we seek expansions which hold in $t$-regions complementary to $\mathbf{T}(\arg \mu)$ when $|\arg \mu| \leq \frac{1}{2} \pi$ (cf. sec 5).

In (8.11) replace $\mu$ by $\mu e^{i \pi}$. Then using (5.24) we obtain

$$
\begin{aligned}
U\left(-\frac{1}{2} \mu^{2},-\mu t \sqrt{ } 2\right) \sim 2 \pi^{\frac{1}{2}} e^{i \pi\left(\frac{1}{2} \mu^{2}-\frac{1}{6}\right)} \mu^{\frac{1}{3}} g(\mu) & \left(\frac{\zeta}{t^{2}-1}\right)^{\frac{1}{4}} \\
\times & {\left[\operatorname{Ai}\left(e^{-\frac{2}{3} \pi i} \mu^{\frac{4}{3}} \zeta\right) \sum_{s=0}^{\infty} \frac{A_{s}(\zeta)}{\mu^{4 s}}+\frac{\operatorname{Ai}^{\prime}\left(e^{-\frac{2}{3} \pi i} \mu^{\frac{4}{3}} \zeta\right)}{e^{\frac{2}{3} \pi i} \mu^{\frac{8}{3}}} \sum_{s=0}^{\infty} \frac{B_{s}(\zeta)}{\mu^{4 s}}\right], }
\end{aligned}
$$


when

$$
-\pi+\epsilon \leq \theta \leq-\epsilon, \quad t \epsilon \mathbf{T}(\theta+\pi) .
$$

In particular, we see from (7.15) and (5.3) that (9.1) holds when

Similarly,

$$
-\frac{1}{2} \pi \leq \theta \leq-\epsilon, \quad t \epsilon C \mathbf{V}_{3}(\theta+\pi)=C \mathbf{V}_{1}(\theta)
$$

$U\left(-\frac{1}{2} \mu^{2},-\mu t \sqrt{ } 2\right) \sim 2 \pi^{\frac{1}{2}} e^{-i \pi\left(\frac{1}{2} \mu^{2}-\frac{1}{6}\right)} \mu^{\frac{1}{3}} g(\mu)\left(\frac{\zeta}{t^{2}-1}\right)^{\frac{1}{4}}$

when

$$
\times\left[\operatorname{Ai}\left(e^{\frac{2}{3} \pi i} \mu^{\frac{4}{3}} \zeta\right) \sum_{s=0}^{\infty} \frac{A_{s}(\zeta)}{\mu^{4 s}}+\frac{\operatorname{Ai}^{\prime}\left(e^{\frac{2}{3} \pi i} \mu^{\frac{4}{3}} \zeta\right)}{e^{-\frac{2}{3} \pi i} \mu^{\frac{8}{3}}} \sum_{s=0}^{\infty} \frac{B_{s}(\zeta)}{\mu^{4 s}}\right]
$$

$$
\epsilon \leq \theta \leq \frac{1}{2} \pi, \quad t \in C \mathbf{V}_{3}(\theta)
$$

The expansions (9.1) and (9.4) are of course the expansions for $U\left(-\frac{1}{2} \mu^{2}, \mu t \sqrt{ } 2\right)$ in terms of Airy functions valid at the turning point $t=-1$. Combined with (8.11) they cover the whole of the $t$-plane when $\epsilon \leq|\theta| \leq \frac{1}{2} \pi$.

When $|\theta| \leq \epsilon$ we cannot expect to be able to represent the function $U\left(-\frac{1}{2} \mu^{2}, \mu t \sqrt{ } 2\right)$ in $\mathbf{V}_{2}(\theta)=C \mathbf{T}(\theta)$ by means of a single Airy function, because as $|t| \rightarrow \infty$ in this region $U\left(-\frac{1}{2} \mu^{2}, \mu t \sqrt{ } 2\right)$ becomes exponentially small or exponentially large according as $\mu^{2}$ is or is not an odd positive integer (cf. (2.7)).

To deal with this remaining case we use the connection formula

$$
\begin{array}{r}
U\left(-\frac{1}{2} \mu^{2},-\mu t \sqrt{ } 2\right)-\sin \frac{1}{2} \pi \mu^{2} \cdot U\left(-\frac{1}{2} \mu^{2}, \mu t \sqrt{ } 2\right)=\left(\frac{\pi}{2}\right)^{\frac{1}{2}} \frac{e^{-i \pi\left(\frac{1}{4} \mu^{2}+\frac{1}{4}\right)}}{\Gamma\left(\frac{1}{2}-\frac{1}{2} \mu^{2}\right)} U\left(\frac{1}{2} \mu^{2},-i \mu t \sqrt{ } 2\right) \\
+\left(\frac{\pi}{2}\right)^{\frac{1}{2}} \frac{e^{i \pi\left(\frac{1}{4} \mu^{2}+\frac{1}{4}\right)}}{\Gamma\left(\frac{1}{2}-\frac{1}{2} \mu^{2}\right)} U\left(\frac{1}{2} \mu^{2}, i \mu t \sqrt{ } 2\right)
\end{array}
$$

obtained in an obvious manner from (5.26). Replacing $\mu$ by $\mu e^{-\frac{1}{2} i \pi}$ in (8.11) and using the first of eqs (5.25), we find

$$
\begin{aligned}
\left(\frac{\pi}{2}\right)^{\frac{1}{2}} \frac{e^{-i \pi\left(\frac{1}{4} \mu^{2}+\frac{1}{4}\right)}}{\Gamma\left(\frac{1}{2}-\frac{1}{2} \mu^{2}\right)} U\left(\frac{1}{2} \mu^{2},-i \mu t \sqrt{ } 2\right) \sim 2 \pi^{\frac{1}{2}} e^{-\frac{1}{6} \pi i} \mu^{\frac{1}{3}} \cos \frac{1}{2} \pi \mu^{2} \cdot g(\mu)\left(\frac{\zeta}{t^{2}-1}\right)^{\frac{1}{4}} & \\
\times & {\left[\operatorname{Ai}\left(e^{-\frac{2}{3} \pi i} \mu^{\frac{4}{3}} \zeta\right) \sum_{s=0}^{\infty} \frac{A_{s}(\zeta)}{\mu^{4 s}}+\frac{\operatorname{Ai}^{\prime}\left(e^{-\frac{2}{3} \pi i} \mu^{\frac{4}{3}} \zeta\right)}{e^{\frac{2}{3} \pi i} \mu^{\frac{8}{3}}} \sum_{s=0}^{\infty} \frac{B_{s}(\zeta)}{\mu^{4 s}}\right] }
\end{aligned}
$$

We substitute this result and its conjugate form on the right of (9.6), and on the left we substitute (8.11). Using the eq [3]

$$
\operatorname{Bi}\left(\mu^{\frac{4}{3}} \zeta\right)=e^{-\frac{1}{6} \pi i} \operatorname{Ai}\left(e^{-\frac{2}{3} \pi i} \mu^{\frac{4}{3}} \zeta\right)+e^{\frac{1}{6} \pi i} \operatorname{Ai}\left(e^{\frac{2}{3} \pi i} \mu^{\frac{4}{3}} \zeta\right),
$$

and its differentiated form, we obtain

$$
\begin{gathered}
U\left(-\frac{1}{2} \mu^{2},-\mu t \sqrt{2}\right) \sim 2 \pi^{\frac{1}{2}} \mu^{\frac{1}{3}} g(\mu)\left(\frac{\zeta}{t^{2}-1}\right)^{\frac{1}{4}} \\
+\sin \frac{1}{2} \pi \mu^{2}\left\{\operatorname{Ai}\left(\mu^{\left.\frac{4}{3} \zeta\right)} \sum_{s=0}^{\infty} \frac{A_{s}(\zeta)}{\mu^{4 s}}+\frac{\operatorname{Ai}^{\prime}\left(\mu^{\frac{4}{3}} \zeta\right)}{\mu^{\frac{8}{3}}} \sum_{s=0}^{\infty} \frac{B_{s}(\zeta)}{\mu^{4 s}}\right\}\right. \\
\left.+\cos \frac{1}{2} \pi \mu^{2}\left\{\operatorname{Bi}\left(\mu^{\frac{4}{3}} \zeta\right) \sum_{s=0}^{\infty} \frac{A_{s}(\zeta)}{\mu^{4 s}}+\frac{\operatorname{Bi}^{\prime}\left(\mu^{\frac{4}{3}} \zeta\right)}{\mu^{\frac{8}{3}}} \sum_{s=0}^{\infty} \frac{B_{s}(\zeta)}{\mu^{4 s}}\right\}\right]
\end{gathered}
$$

This is valid when $|\theta| \leq \frac{1}{2} \pi-\epsilon$ in the $t$-region common to the domains $\mathbf{T}(\theta), \mathbf{T}\left(\theta-\frac{1}{2} \pi\right)$, and 
$\mathbf{T}\left(\theta+\frac{1}{2} \pi\right)$. Using (7.15) and (5.2), we see that this means (9.7) is valid when

$$
|\theta| \leq \frac{1}{2} \pi-\epsilon, \quad t \epsilon C \mathbf{V}_{2}(\theta)
$$

This is the required result. We note that when $\mu^{2}$ is an odd integer the terms in $\mathrm{Bi}, \mathrm{Bi}^{\prime}$ in (9.7) vanish, as we require from a consideration of the behavior of $U\left(-\frac{1}{2} \mu^{2}, \mu t \sqrt{ } 2\right)$ as $|t| \rightarrow \infty$ in the sector $\mathbf{V}_{2}(\theta)$. We may also verify without difficulty that (9.7) agrees with (9.1) and (9.4) in their common $t$-regions of validity when $-\frac{1}{2} \pi+\epsilon \leq \theta \leq-\epsilon$ and $\epsilon \leq \theta \leq \frac{1}{2} \pi-\epsilon$, respectively.

\section{Summary of Expansions in Terms of Airy Functions}

The fundamental expansion of this kind is (8.11). It is valid for large $|\mu|$ uniformly with respect to $\theta \equiv \arg \mu$ and $t$, when $-\pi+\epsilon \leq \theta \leq \pi-\epsilon$ and $t$ lies in the domain $\mathbf{T}(\theta)$, defined by (7.15) and illustrated in figure 7 . The function $g(\mu)$ is calculable from either of the asymptotic expansions (6.1) and (6.2). The function $\zeta(t)$ is given by (7.4), that branch being chosen which is continuous in $\mathbf{T}(\theta)$ and positive when $t>1$; similarly the branch of $\left\{\zeta /\left(t^{2}-1\right)\right\}^{\frac{1}{4}}$ is continuous in $\mathbf{T}(\theta)$ and positive when $t>1$. The coefficients $A_{s}(\zeta)$ and $B_{s}(\zeta)$ are given by eqs (8.12) ; they satisfy the recurrence relations (7.12) and the eqs (8.3). They are regular functions of $\zeta$ in the $\zeta$-map of $\mathbf{T}(\theta)$ and real when $\zeta$ lies on the real axis to the right of the point of affix $-\left(\frac{3}{4} \pi\right)^{\frac{2}{3}}$.

Other asymptotic expansions for $U\left(-\frac{1}{2} \mu^{2}, \mu t \sqrt{ } 2\right)$ are $(9.1),(9.4)$, and $(9.7)$; they are valid with the conditions (9.3), (9.5), and (9.8), respectively. The combination of these expansions and (8.11) covers the whole $t$-plane when $|\theta| \leq \frac{1}{2} \pi$.

The expansion for the derivative $U^{\prime}\left(-\frac{1}{2} \mu^{2}, \mu t \sqrt{2}\right)$ corresponding to (8.11) is (8.15), and holds under the same conditions. The coefficients $C_{s}(\zeta)$ and $D_{s}(\zeta)$ are given by $(8.17)$ and (8.19). The derivative expansions corresponding to (9.1), (9.4), and (9.7) are not recorded, but they can be obtained immediately by analogy with (8.15). They are valid under the same conditions as the parent formulas.

Approximations for parabolic cylinder functions of large orders which hold in regions containing one of the turning points have been given by Watson ([12], sec. 17), Schwid ([10], sec. 6), Erdélyi, Kennedy, and McGregor [2], and Kazarınoff [15].

Watson derives asymptotic expansions in terms of elementary functions for the functions $D_{n}(z \pm 2 \sqrt{ } n) \equiv U\left(-n-\frac{1}{2}, z \pm 2 \sqrt{ } n\right)$ as $|n| \rightarrow \infty$, with the conditions $|\arg n|<\frac{1}{2} \pi$ and $z=o\left(|n|^{-\frac{1}{6}}\right)$. In the present notation these conditions correspond to $|\arg \mu|<\frac{1}{4} \pi$ and $t \mp 1=o\left(|\mu|^{-\frac{4}{3}}\right)$. Watson's expansions are analogous to Mieissel's expansions for Bessel functions of large order and may be obtained from the present uniform expansions by suitable re-expansion (cf. [8], sec. 6).

Schwid gives approximations in terms of Bessel functions of order one-third (Airy functions) which are valid in the neighborhood $t-1=O\left(|\mu|^{-2}\right)$ as $|\mu| \rightarrow \infty$, using the present notation. This neighborhood is complementary to the region of validity of Schwid's approximations in terms of elementary functions, described in section 6 . No investigation is made of the validity of the Bessel function approximations outside the neighborhood.

The approach of Erdélyi, Kennedy, and McGregor is similar to the one we have used. These writers obtain a set of asymptotic approximations in terms of Airy functions, to solutions of eq (1.1) when $\mu$ and $t$ are both complex (using the present notation). The results are contained in those given in the present paper; the principal ways in which they have been extended and improved upon are as follows.

First, the approximations apply to fixed values of arg $\mu$, no investigation is made of their uniform validity with respect to arg $\mu$.

Secondly, the approximations are established with the condition $|\arg \mu| \leq \frac{1}{4} \pi$; outside this interval connection formulas are to be used. This leads to complexity in the number of results; eleven are given compared with the four we have found to be sufficient.

Finally, only the leading terms of the asymptotic expansions are found; no terms involving the first derivative of the Airy functions appear in the approximations. An effect of this is 
to exclude regions in the $t$-plane containing the zeros of the Airy functions. No special treatment of such regions is required in the present theory.

Kazarinoff's paper (which has appeared since the present paper was written) contains a brief investigation of the extension of the results of Erdélyi, Kennedy, and McGregor to include uniformity with respect to $\arg \mu$ and higher terms in the asymptotic expansions. The results are rather more complicated and less complete than those given here.

\section{Part 4. Results for Real Variables}

In this part we suppose $\mu, t, a$, and $x$ to denote real variables except where otherwise indicated; $\mu$ is always large and positive. The variable $x$ is not to be confused with the complex function $x(t)$ introduced in parts 2 and 3 .

\section{The Equation $w^{\prime \prime}=\left(\frac{1}{4} x^{2}+a\right) w$}

The principal solutions of this equation are $U(a, x), U(a,-x), V(a, x)$, and $\bar{U}(a, x)$, defined in section 2 .

(i) a positive. When $a \rightarrow+\infty$ the differential equation has no turning points on the real $x$-axis. The expansions of greatest interest are therefore those in terms of elementary functions, and we shall not consider the forms of the Airy expansions.

In (4.3) replace $\mu, t$ by $i \mu$, - it, respectively. Then using (4.8), we obtain

$$
U\left(\frac{1}{2} \mu^{2}, \mu t \sqrt{ } 2\right) \sim g\left(\mu e^{\frac{1}{2} i \pi}\right) \frac{e^{\mu 2 \xi(-i t)}}{\left(-t^{2}-1\right)^{\frac{1}{4}}} \sum_{s=0}^{\infty}(-)^{s} \frac{u_{s}(-i t)}{\left(-t^{2}-1\right)^{\frac{3}{2} s}} \frac{1}{\mu^{2 s}}
$$

as $\mu \rightarrow+\infty$. In the complex plane the region of validity of this expansion is $e^{\frac{1}{2} i \pi} \mathbf{S}\left(\frac{1}{2} \pi\right)$, and from figure $2(\mathrm{c})$ it is clear that this region includes the whole of the real $t$-axis.

We have now to interpret the branches of the various functions.

From (6.1) and (6.2) we derive

$$
g\left(\mu e^{\frac{1}{2} i \pi}\right)=e^{-i \pi\left(\frac{1}{4}+\frac{1}{4} \mu^{2}\right)} \bar{g}(\mu),
$$

where the function $\bar{g}(\mu)$ is real and has the expansions

$$
\begin{gathered}
\frac{1}{\bar{g}(\mu)} \sim 2^{-\frac{1}{4} \mu^{2}+\frac{1}{4}} e^{-\frac{1}{4} \mu^{2}} \mu^{\frac{1}{2} \mu^{2}+\frac{1}{2}}\left\{1-\sum_{s=0}^{\infty} \frac{g_{2 s+1}}{\mu^{4 s+2}}\right\}, \\
\bar{g}(\mu) \sim 2^{\frac{1}{4} \mu^{2}-\frac{1}{4}} e^{\frac{1}{4} \mu^{2}} \mu^{-\frac{1}{2} \mu^{2}-\frac{1}{2}}\left\{1+\frac{1}{2} \sum_{s=1}^{\infty}(-)^{s} \frac{\gamma_{s}}{\left(\frac{1}{2} \mu^{2}\right)^{s}}\right\},
\end{gathered}
$$

as $\mu \rightarrow+\infty$. Comparison of (11.4) with (2.27) shows incidentally that

$$
\bar{g}(\mu) \simeq 2^{\frac{1}{4} \mu^{2}-\frac{5}{4}} e^{\frac{1}{4} \mu^{2}} \mu^{-\frac{1}{2} \mu^{2}-\frac{1}{2}}+\frac{\pi^{\frac{1}{2}} 2^{-\frac{1}{4} \mu^{2}-\frac{3}{4}} e^{-\frac{1}{4} \mu^{2}} \mu^{\frac{1}{2} \mu^{2}-\frac{1}{2}}}{\Gamma\left(\frac{1}{2}+\frac{1}{2} \mu^{2}\right)},
$$

where the symbol $\simeq$ is defined in section 5 .

Next, when $t$ lies on the negative imaginary axis of figure 2(c), $\xi(t)$ lies on the straight line $\mathrm{O}^{\prime} \mathrm{C}^{\prime}$ shown in the third diagram of figure 1 . Hence

$$
\xi(-i t)=\frac{1}{4} i \pi-\bar{\xi}(t),
$$

where $\bar{\xi}(t)$ is positive when $t>0$. From (3.4) we find

$$
\bar{\xi}(t)=\int_{0}^{t}\left(t^{2}+1\right)^{\frac{1}{2}} d t=\frac{1}{2} t\left(t^{2}+1\right)^{\frac{1}{2}}+\frac{1}{2} \ln \left\{t+\left(t^{2}+1\right)^{\frac{1}{2}}\right\},
$$

the branches here baving their principal values. 
Lastly, we have

$$
\left(-t^{2}-1\right)^{\frac{1}{4}}=e^{-\frac{1}{4} i \pi}\left(t^{2}+1\right)^{\frac{1}{4}}, \quad\left(-t^{2}-1\right)^{\frac{3}{2} s}=e^{-\frac{3}{2} s i \pi}\left(t^{2}+1\right)^{\frac{3}{2} s},
$$

where the functions on the right have their principal values.

Substituting in (11.1) by means of (11.2), (11.6), (11.8), and the relation

$$
\bar{u}_{s}(t) \equiv i^{s} u_{s}(-i t) \text {, }
$$

we obtain the required result in real form, given by

$$
U\left(\frac{1}{2} \mu^{2}, \mu t \sqrt{ } 2\right) \sim \bar{g}(\mu) \frac{e^{-\mu^{2} \bar{\xi}(t)}}{\left(t^{2}+1\right)^{\frac{1}{4}}} \sum_{s=0}^{\infty} \frac{\bar{u}_{s}(t)}{\left(t^{2}+1\right)^{\frac{3}{2} s}} \frac{1}{\mu^{2 s}} \quad(-\infty<t<\infty),
$$

uniformly with respect to $t$. Explicit expressions for the coefficients $\bar{u}_{s}(t)$, obtained from (4.11), are

$$
\left.\begin{array}{l}
\bar{u}_{0}(t)=1, \quad \bar{u}_{1}(t)=\left(-t^{3}-6 t\right) / 24, \quad \bar{u}_{2}(t)=\left(9 t^{4}+249 t^{2}-145\right) / 1152, \\
\bar{u}_{3}(t)=\left(4042 t^{9}+18189 t^{7}+28287 t^{5}-151995 t^{3}+259290 t\right) / 414720 .
\end{array}\right\}
$$

The corresponding result for the derivative is

$$
U^{\prime}\left(\frac{1}{2} \mu^{2}, \mu t \sqrt{ } 2\right) \sim-\frac{\mu}{\sqrt{ } 2} \bar{g}(\mu)\left(t^{2}+1\right)^{\frac{1}{4}} e^{-\mu^{2} \bar{\xi}(t)} \sum_{s=0}^{\infty} \frac{\bar{v}_{s}(t)}{\left(t^{2}+1\right)^{\frac{3}{2} s}} \frac{1}{\mu^{2 s}},
$$

valid with the same conditions. Here

$$
\bar{v}_{s}(t) \equiv i^{s} v_{s}(-i t) .
$$

Explicitly,

$$
\left.\begin{array}{l}
\bar{v}_{0}(t)=1, \quad \bar{v}_{1}(t)=\left(-t^{3}+6 t\right) / 24, \quad \bar{v}_{2}(t)=\left(-15 t^{4}-327 t^{2}+143\right) / 1152, \\
\bar{v}_{3}(t)=\left(4042 t^{9}+18189 t^{7}+36387 t^{5}+238425 t^{3}-259290 t\right) / 414720,
\end{array}\right\}
$$

(cf. (4.13) to $(4.16))$.

Since $U(a, x)$ and $U(a,-x)$ comprise a satisfactory pair of solutions of the differential equation when $a$ is positive, it is unnecessary to give the corresponding expansions for the solutions $V(a, x)$ and $\bar{U}(a, x)$.

(ii) a negative; expansions in terms of elementary functions. For $t \geq 1+\delta$, the expansion of $U\left(-\frac{1}{2} \mu^{2}, \mu t \sqrt{ } 2\right)$ is given in real form by $(4.3)^{3}$. In this expansion $g(\mu)$ is calculable from $(6.1)$, $(6.2)$, or the relation

$$
g(\mu) \simeq 2^{-\frac{1}{4} \mu^{2}-\frac{5}{4}} e^{-\frac{1}{4} \mu^{2}} \mu^{\frac{1}{2} \mu^{2}-\frac{1}{2}}+\pi^{-\frac{1}{2}} 2^{\frac{1}{4} \mu^{2}-\frac{7}{4}} e^{\frac{1}{4} \mu^{2}} \mu^{-\frac{1}{2} \mu^{2}-\frac{1}{2}} \Gamma\left(\frac{1}{2}+\frac{1}{2} \mu^{2}\right),
$$

and $\xi, \mathscr{A}_{s}(\xi)$, by use of (3.4), (4.8), and (4.11), all fractional powers now taking their principal values.

When $-1+\delta \leq t \leq 1-\delta$, the expansion of $U\left(-\frac{1}{2} \mu^{2}, \mu t \sqrt{ } 2\right)$ is given by (5.11). Here $\tilde{g}(\mu)$ is identical with $g(\mu)$ and $\eta$ is given by (5.9).

For the remaining part of the real axis we have the expansion (5.28). In the present circumstances the function $\xi(t)$ is positive and bounded away from zero. Hence the contribution of the series $i \cos \frac{1}{2} \pi \mu^{2} \cdot e^{-\mu^{2} \xi} \sum \mathscr{A}_{s}(\xi) \mu^{-2 s}$ is always asymptotically negligible compared with that of $2 \cos \frac{1}{2} \pi \mu^{2} \cdot e^{\mu \xi \xi} \sum(-)^{s} \mathscr{A}_{s}(\xi) \mu^{-2 s}$. Thus (5.28) reduces to

$U\left(-\frac{1}{2} \mu^{2},-\mu t \sqrt{ } 2\right) \sim \frac{g(\mu)}{\left(t^{2}-1\right)^{\frac{1}{4}}}\left[2 \cos \frac{1}{2} \pi \mu^{2} \cdot e^{\mu^{2} \xi} \sum_{s=0}^{\infty}(-)^{s} \frac{\mathscr{A}_{s}(\xi)}{\mu^{2 s}}+\sin \frac{1}{2} \pi \mu^{2} \cdot e^{-\mu^{2} \xi} \sum_{s=0}^{\infty} \frac{\mathscr{A}_{s}(\xi)}{\mu^{2 s}}\right]$

\footnotetext{
${ }^{3}$ For convenience the symbol $\delta^{\prime}$ of parts 2 and 3 , denoting an arbitrary fixed positive number, is replaced here by $\delta$.
} 
valid when $t \geq 1+\delta$. The functions $g(\mu), \xi$ and $\mathscr{A}_{s}(\xi)$ are the same as in (4.3), discussed above.

The corresponding expansions for the second solution $\bar{U}(a, x)$ are of interest, particularly when $x \geq 0$. It is not possible to obtain them trivially by substituting in the connection formula (2.12) the expansions we have just obtained for $U(a, x)$ and $U(a,-x)$, because although cancellation of terms in the asymptotic series takes place, we cannot assert immediately that the remainder terms implied in these series also cancel. To put the results on a sound basis we substitute the expansions (5.5) and (5.6) in the formula

$\bar{U}\left(-\frac{1}{2} \mu^{2}, \mu t \sqrt{ } 2\right)=(2 \pi)^{-\frac{1}{2}} \Gamma\left(\frac{1}{2}+\frac{1}{2} \mu^{2}\right)\left[e^{-i \pi\left(\frac{1}{4}+\frac{1}{4} \mu^{2}\right)} U\left(\frac{1}{2} \mu^{2},-i \mu t \sqrt{ } 2\right)+e^{i \pi\left(\frac{1}{4}+\frac{1}{4} \mu^{2}\right)} U\left(\frac{1}{2} \mu^{2}, i \mu t \sqrt{ } 2\right)\right]$,

obtained from (2.5) and (2.12).

When $t \geq 1+\delta$ the branches of $\xi(t)$ and $\mathscr{A}_{s}(\xi)$ in both expansions are the principal ones. With the aid of the relation

$$
(2 \pi)^{-\frac{1}{2}} \Gamma\left(\frac{1}{2}+\frac{1}{2} \mu^{2}\right) e^{\mp i \pi\left(\frac{1}{4}+\frac{1}{4} \mu^{2}\right)} g\left(\mu e^{\mp \frac{1}{2} i \pi}\right) \simeq g(\mu),
$$

proved by use of $(2.22),(5.7),(5.20)$, and (6.2), we deduce that

$$
\bar{U}\left(-\frac{1}{2} \mu^{2}, \mu t \sqrt{ } 2\right) \sim 2 g(\mu) \frac{e^{\mu 2 \xi}}{\left(t^{2}-1\right)^{\frac{1}{4}}} \sum_{s=0}^{\infty}(-)^{s} \frac{u_{s}(t)}{\left(t^{2}-1\right)^{\frac{3}{2} s}} \frac{1}{\mu^{2 s}} \quad(t \geq 1+\delta) .
$$

When $-1+\delta \leq t \leq 1-\delta$, we interpret the branches of the many-valued functions by use of (5.8) and (5.10). With the aid of (11.18) we obtain on reduction

$$
\bar{U}\left(-\frac{1}{2} \mu^{2}, \mu t \sqrt{ } 2\right) \sim \frac{2 g(\mu)}{\left(1-t^{2}\right)^{\frac{1}{4}}}\left[\cos \left(\mu^{2} \eta+\frac{1}{4} \pi\right) \sum_{s=0}^{\infty} \frac{(-)^{s} u_{2 s}(t)}{\left(1-t^{2}\right)^{3 s} \mu^{4 s}}-\sin \left(\mu^{2} \eta+\frac{1}{4} \pi\right) \sum_{s=0}^{\infty} \frac{(-)^{s} u_{2 s+1}(t)}{\left(1-t^{2}\right)^{3 s+\frac{3}{2}} \mu^{4 s+2}}\right]
$$

valid when $-1+\delta \leq t \leq 1-\delta$.

The expansions for the derivatives corresponding to the expansions obtained in this subsection can be written down immediately by use of (6.3), or by analogy with (5.23).

(iii) a negative; expansions in terms of Airy functions. For $t \geq-1+\delta$ the expansions of $U\left(-\frac{1}{2} \mu^{2}, \mu t \sqrt{ } 2\right)$ and $U\left(-\frac{1}{2} \mu^{2},-\mu t \sqrt{ } 2\right)$ are given by (8.11) and (9.7), respectively. In these expansions $\zeta(t)$ is given by (7.4) and is real when $t>-1$; a more convenient form of this relation when $-1<t<1$ is

$$
\frac{2}{3}(-\zeta)^{\frac{3}{2}}=\int_{t}^{1}\left(1-t^{2}\right)^{\frac{1}{2}} d t=\frac{1}{2} \cos ^{-1} t-\frac{1}{2} t\left(1-t^{2}\right)^{\frac{1}{2}}=\eta(t)
$$

(cf. (5.9)). The coefficients $A_{s}(\zeta)$ and $B_{s}(\zeta)$ are real and given by (8.12), (8.13), and (4.8)

An expansion for the solution $\bar{U}(a, x)$ may be derived by use of (11.17), as in the case of the exponential-type expansions. The result is

$$
\bar{U}\left(-\frac{1}{2} \mu^{2}, \mu t \sqrt{ } 2\right) \sim 2 \pi^{\frac{1}{2}} \mu^{\frac{1}{3}} g(\mu)\left(\frac{\zeta}{t^{2}-1}\right)^{\frac{1}{4}}\left[\operatorname{Bi}\left(\mu^{\frac{4}{3}} \zeta\right) \sum_{s=0}^{\infty} \frac{A_{s}(\zeta)}{\mu^{4 s}}+\frac{\mathrm{Bi}^{\prime}\left(\mu^{\frac{4}{3}} \zeta\right)}{\mu^{\frac{8}{3}}} \sum_{s=0}^{\infty} \frac{B_{s}(\zeta)}{\mu^{4 s}}\right]
$$

valid when $t \geq-1+\delta$.

The corresponding expansion for $\bar{U}^{\prime}\left(-\frac{1}{2} \mu^{2}, \mu t \sqrt{ } 2\right)$ may be written down by analogy with (8.15).

(iv) Hermite polynomials of large degree. Expansions for the Hermite polynomial $H_{n}(x)$

4. More convenient expressions when $-1<t<1$ are given by (13.4) and (13.5). 
when $n$ is large can be obtained from the results in subsections (ii) and (iii) above by use of the equation

$$
H_{\frac{1}{2} \mu^{2}-\frac{1}{2}}(\mu t)=2^{\frac{1}{4} \mu^{2}-\frac{1}{4}} e^{\frac{1}{2} \mu^{2} t^{2}} U\left(-\frac{1}{2} \mu^{2}, \mu t \sqrt{ } 2\right)
$$

Thus (4.3) and (5.11) yield expansions which hold uniformly in the ranges $x \geq(1+\delta)(2 n+1)^{\frac{1}{2}}$ and $-(1-\delta)(2 n+1)^{\frac{1}{2}} \leq x \leq(1-\delta)(2 n+1)^{\frac{1}{2}}$, respectively. An expansion uniformly valid throughout the range $x \geq-(1-\delta)(2 n+1)^{\frac{1}{2}}$ is furnished by the Airy expansion (8.11).

\section{The Equation $w^{\prime \prime}=\left(a-\frac{1}{4} x^{2}\right) w$}

(i) a positive; expansions in terms of elementary functions. From (2.16), (2.17) and (2.18), we have

$$
k^{-\frac{1}{2}} W\left(\frac{1}{2} \mu^{2}, \mu t \sqrt{ } 2\right)+i k^{\frac{1}{2}} W\left(\frac{1}{2} \mu^{2},-\mu t \sqrt{ } 2\right)=2^{\frac{1}{2}} e^{\frac{1}{8} \pi \mu^{2}} e^{i\left(\frac{1}{2} \phi_{2}+\frac{1}{8} \pi\right)} U\left(\frac{1}{2} i \mu^{2}, e^{-\frac{1}{4} \pi i} \mu t \sqrt{ } 2\right),
$$

where

$$
k=\left(1+e^{\pi \mu^{2}}\right)^{\frac{1}{2}}-e^{\frac{1}{2} \pi \mu^{2}}, \quad e^{\frac{1}{2} i \phi_{2}}= \pm\left\{\Gamma\left(\frac{1}{2}+\frac{1}{2} i \mu^{2}\right) /\left|\Gamma\left(\frac{1}{2}+\frac{1}{2} i \mu^{2}\right)\right|\right\}^{\frac{1}{2}} .
$$

For large $\mu$,

$$
k=\frac{1}{2} e^{-\frac{1}{2} \pi \mu^{2}}+O\left(e^{-\frac{3}{2} \pi \mu^{2}}\right), \quad e^{\frac{1}{2} l \phi_{2}}=e^{-\frac{1}{4} i \mu^{2}}\left(\frac{1}{2} \mu^{2}\right)^{\frac{1}{4} i \mu^{2}}\left\{1+O\left(\mu^{-2}\right)\right\} .
$$

In (4.3) replace $\mu$ by $\mu e^{-\frac{1}{4} \pi i}$. Then we obtain

$$
U\left(\frac{1}{2} i \mu^{2}, e^{-\frac{1}{4} \pi i} \mu t \sqrt{ } 2\right) \sim g\left(\mu e^{-\frac{1}{4} \pi i}\right) \frac{e^{i \mu^{2} \xi}}{\left(t^{2}-1\right)^{\frac{1}{4}}} \sum_{s=0}^{\infty} i^{s} \frac{u_{s}(t)}{\left(t^{2}-1\right)^{\frac{3}{2} s}} \frac{1}{\mu^{2 s}}
$$

as $\mu \rightarrow+\infty$. The $t$-region of validity of this expansion, $\mathbf{S}\left(-\frac{1}{4} \pi\right)$, includes the segments $t \geq 1+\delta$ and $-1+\delta \leq t \leq 1-\delta$ of the real axis (cf. fig. $2(\mathrm{~b})$ ).

When $\mu \rightarrow+\infty$, we have from (12.2) and (2.22)

$$
e^{\frac{1}{2} i \phi_{2}} \sim e^{-\frac{1}{4} i \mu^{2}}\left(\frac{1}{2} \mu^{2}\right)^{\frac{1}{4} i \mu^{2}}\left[\sum_{s=0}^{\infty} \frac{\gamma_{s}}{\left(\frac{1}{2} i \mu^{2}\right)^{s}}\right]^{\frac{1}{2}}\left|\sum_{s=0}^{\infty} \frac{\gamma_{s}}{\left(\frac{1}{2} i \mu^{2}\right)^{s}}\right|^{-\frac{1}{2}}
$$

the choice of ambiguous sign on taking the square root being resolved by use of (12.3). From (6.1)

$$
g\left(\mu e^{-\frac{1}{4} \pi i}\right) \sim 2^{\frac{1}{4} \mu^{2}-\frac{1}{4}} e^{\frac{1}{4} \mu^{2}} e^{\frac{1}{8} \pi i-\frac{1}{8} \pi \mu^{2}} \mu^{-\frac{1}{2} i \mu^{2}-\frac{1}{2}}(1+i L)^{-1},
$$

where the symbol $L$ denotes the asymptotic series

$$
L \equiv \sum_{s=0}^{\infty}(-)^{s} \frac{g_{2 s+1}}{\mu^{4 s+2}}
$$

Further from (5.21), with $\mu$ replaced by $\mu e^{\frac{1}{4} \pi i}$, we have

$$
\sum_{s=0}^{\infty} \frac{\gamma_{s}}{\left(\frac{1}{2} i \mu^{2}\right)^{s}} \sim \frac{2}{1-i L}-1=\frac{1+i L}{1-i L} .
$$

Introducing a function $l(\mu)$, defined by

$$
l(\mu) \equiv 2^{\frac{1}{2}} e^{\frac{1}{8} \pi \mu^{2}} e^{i\left(\frac{1}{2} \phi_{2}-\frac{1}{8} \pi\right)} g\left(\mu e^{-\frac{1}{4} \pi i}\right),
$$

and using (12.5), (12.6) and (12.8), we find that

$$
l(\mu) \sim \frac{2^{\frac{1}{4}}}{\mu^{\frac{1}{2}}}\left(\frac{1+i L}{1-i L}\right)^{\frac{1}{2}}\left|\frac{1+i L}{1-i L}\right|^{-\frac{1}{2}} \frac{1}{1+i L} \sim \frac{2^{\frac{1}{4}}}{\mu^{\frac{1}{2}}} \frac{1}{\left(1+L^{2}\right)^{\frac{1}{2}}} .
$$


Thus $l(\mu)$ is a real function, having an asymptotic expansion of the form

$$
l(\mu) \sim \frac{2^{\frac{1}{4}}}{\mu^{\frac{1}{2}}} \sum_{s=0}^{\infty} \frac{l_{s}}{\mu^{4 s}} \quad \text { as } \mu \rightarrow+\infty .
$$

From (12.10), (12.7), and (4.12), we find

$$
l_{0}=1, \quad l_{1}=-\frac{1}{2} g_{1}^{2}=-\frac{1}{1152}, \quad l_{2}=g_{1} g_{3}+\frac{3}{8} g_{1}^{4}=-\frac{16123}{39813120} .
$$

We are now in a position to combine (12.1) with (12.4). When $t \geq 1+\delta$ each of the functions $\xi(t),\left(t^{2}-1\right)^{\frac{1}{4}}$ and $\left(t^{2}-1\right)^{\frac{3}{2} s}$ is positive. Substituting by means of $(12.9)$ and the first of (12.3), and equating real and imaginary parts, we find

$W\left(\frac{1}{2} \mu^{2}, \mu t \sqrt{ } 2\right) \sim \frac{2^{-\frac{1}{2}} e^{-\frac{1}{4} \pi \mu^{2}} l(\mu)}{\left(t^{2}-1\right)^{\frac{1}{4}}}\left[\cos \left(\mu^{2} \xi+\frac{1}{4} \pi\right) \sum_{s=0}^{\infty} \frac{(-)^{s} u_{2 s}(t)}{\left(t^{2}-1\right)^{3 s} \mu^{4 s}}-\sin \left(\mu^{2} \xi+\frac{1}{4} \pi\right) \sum_{s=0}^{\infty} \frac{(-)^{s} u_{2 s+1}(t)}{\left(t^{2}-1\right)^{3 s+\frac{3}{2}} \mu^{4 s+2}}\right]$,

$$
W\left(\frac{1}{2} \mu^{2},-\mu t \sqrt{ } 2\right) \sim \frac{2^{\frac{1}{2}} e^{\frac{1}{4} \pi \mu^{2}} l(\mu)}{\left(t^{2}-1\right)^{\frac{1}{4}}}\left[\sin \left(\mu^{2} \xi+\frac{1}{4} \pi\right) \sum_{s=0}^{\infty} \frac{(-)^{s} u_{2 s}(t)}{\left(t^{2}-1\right)^{3 s} \mu^{4 s}}+\cos \left(\mu^{2} \xi+\frac{1}{4} \pi\right) \sum_{s=0}^{\infty} \frac{(-)^{s} u_{2 s+1}(t)}{\left(t^{2}-1\right)^{3 s+\frac{3}{2}} \mu^{4 s+2}}\right],
$$

uniformly with respect to $t$ when $t \geq 1+\delta$. The function $l(\mu)$ is calculable from (12.11).

When $-1+\delta \leq t \leq 1-\delta$ the appropriate branches of $\xi$ and $\left(t^{2}-1\right)^{\frac{1}{4}}$ are given by $\xi=-i \eta$ and $\left(t^{2}-1\right)^{\frac{1}{4}}=e^{\frac{1}{2 \pi i}}\left(1-t^{2}\right)^{\frac{1}{4}}$, where $\eta$ is the real function $(5.9)$ and $\left(1-t^{2}\right)^{\frac{1}{4}}$ is positive. Hence we derive

$$
W\left(\frac{1}{2} \mu^{2}, \mu t \sqrt{ } 2\right) \sim \frac{l(\mu)}{2^{\frac{1}{2}} e^{\frac{1}{4} \pi \mu^{2}}} \frac{e^{\mu 2 \eta}}{\left(1-t^{2}\right)^{\frac{1}{4}}} \sum_{s=0}^{\infty} \frac{(-)^{s} u_{s}(t)}{\left(1-t^{2}\right)^{\frac{3}{2} s}} \frac{1}{\mu^{2 s}} \quad(-1+\delta \leq t \leq 1-\delta) .
$$

The expansions for the derivatives corresponding to (12.13), (12.14), and (12.15) may be obtained by term-by-term differentiation with respect to $t$ (cf. (5.23) and (4.13)).

It may be noted that (12.13), (12.14), and (12.15) are respectively equivalent to the formal series (317), (318) and (324) given on pages 84 and 85 of [4] (cf. sec. 6).

(ii) a positive; expansions in terms of Airy functions. In (8.11) replace $\mu$ by $\mu e^{-\frac{1}{4} \pi \imath}$. Then we obtain

$$
\begin{aligned}
U\left(\frac{1}{2} i \mu^{2}, e^{-\frac{1}{4} \pi i} \mu t \sqrt{ } 2\right) \sim & \frac{2 \pi^{\frac{1}{2}} \mu^{\frac{1}{3}}}{e^{\frac{1}{12} \pi i}} g\left(\mu e^{-\frac{1}{4} \pi i}\right)\left(\frac{\zeta}{t^{2}-1}\right)^{\frac{1}{4}} \\
& \times\left[\operatorname{Ai}\left(e^{-\frac{1}{3} \pi} \mu^{\frac{4}{3}} \zeta\right) \sum_{s=0}^{\infty}(-)^{s} \frac{A_{s}(\zeta)}{\mu^{4 s}}+\frac{\operatorname{Ai}^{\prime}\left(e^{-\frac{1}{3} \pi i} \mu^{\frac{4}{3}} \zeta\right)}{e^{-\frac{2}{3} \pi i} \mu^{\frac{8}{3}}} \sum_{s=0}^{\infty}(-)^{s} \frac{B_{s}(\zeta)}{\mu^{4 s}}\right]
\end{aligned}
$$

The $t$-region of validity of this expansion, $\mathbf{T}\left(-\frac{1}{4} \pi\right)$, includes the segment $t \geq-1+\delta$ (cf. fig. $7(b))$. Now from [3]

$$
2 e^{\frac{1}{6} \pi i} \mathrm{Ai}\left(e^{-\frac{1}{3} \pi i} \mu^{\frac{4}{3}} \zeta\right)=\mathrm{Bi}\left(-\mu^{\frac{4}{3}} \zeta\right)+i \mathrm{Ai}\left(-\mu^{\frac{4}{3}} \zeta\right)
$$

Hence substituting (12.16) in (12.1) and equating real parts, we find, with the aid of (12.9) and the first of (12.3),

$W\left(\frac{1}{2} \mu^{2}, \mu t \sqrt{ } 2\right) \sim \frac{\pi^{\frac{1}{2}} \mu^{\frac{1}{3}} l(\mu)}{2^{\frac{1}{2}} e^{\frac{1}{4} \pi \mu^{2}}}\left(\frac{\zeta}{t^{2}-1}\right)^{\frac{1}{4}}\left[\operatorname{Bi}\left(-\mu^{\frac{4}{3}} \zeta\right) \sum_{s=0}^{\infty}(-)^{s} \frac{A_{s}(\zeta)}{\mu^{4 s}}+\frac{\mathrm{Bi}^{\prime}\left(-\mu^{\frac{4}{3}} \zeta\right)}{\mu^{\frac{8}{3}}} \sum_{s=0}^{\infty}(-)^{s} \frac{B_{s}(\zeta)}{\mu^{4 s}}\right](1$

valid when $t \geq-1+\delta$. The corresponding expansion for the derivative is 
$W^{\prime}\left(\frac{1}{2} \mu^{2}, \mu t \sqrt{ } 2\right) \sim-\frac{\pi^{\frac{1}{2}} \mu^{\frac{2}{3}} l(\mu)}{2 e^{\frac{1}{4} \pi \mu^{2}}}\left(\frac{t^{2}-1}{\zeta}\right)^{\frac{1}{4}}$

$$
\times\left[-\frac{\mathrm{Bi}\left(-\mu^{\frac{4}{5}} \zeta\right)}{\mu^{\frac{4}{3}}} \sum_{s=0}^{\infty}(-)^{s} \frac{C_{s}(\zeta)}{\mu^{4 s}}+\mathrm{Bi}^{\prime}\left(-\mu^{\frac{4}{3}} \zeta\right) \sum_{s=0}^{\infty}(-)^{s} \frac{D_{s}(\zeta)}{\mu^{4 s}}\right]
$$

(cf. $(8.15))$.

The expansion for the second solution may be obtained by equating imaginary parts. This gives

$W\left(\frac{1}{2} \mu^{2},-\mu t \sqrt{ } 2\right) \sim \frac{\pi^{\frac{1}{2}} \mu^{\frac{1}{3}} l(\mu)}{2^{-\frac{1}{2}} e^{-\frac{1}{4} \pi \mu^{2}}}\left(\frac{\zeta}{t^{2}-1}\right)^{\frac{1}{4}}\left[\mathrm{Ai}\left(-\mu^{\frac{4}{3}} \zeta\right) \sum_{s=0}^{\infty}(-)^{s} \frac{A_{s}(\zeta)}{\mu^{4 s}}+\frac{\operatorname{Ai}^{\prime}\left(-\mu^{\frac{4}{3}} \zeta\right)}{\mu^{\frac{8}{3}}} \sum_{s=0}^{\infty}(-)^{s} \frac{B_{s}(\zeta)}{\mu^{4 s}}\right]$

However, this result is only proved in this way for $t \geq 1$, i.e., non-negative values of $\zeta$. This is because the error term on truncating the series inside the square brackets at the $m$ th terms is

$$
\left\{\mathrm{Bi}\left(-\mu^{\frac{4}{3}} \zeta\right)+i \mathrm{Ai}\left(-\mu^{\frac{4}{3}} \zeta\right)\right\} O\left(\mu^{-4 m}\right)+\left\{\mathrm{Bi}^{\prime}\left(-\mu^{\frac{4}{5}} \zeta\right)+i \mathrm{Ai}^{\prime}\left(-\mu^{\frac{4}{3}} \zeta\right)\right\}\left(1+|\zeta|^{\frac{1}{2}}\right)^{-1} O\left(\mu^{-4 m-\frac{8}{3}}\right) .
$$

When $\zeta$ is negative the terms in $\mathrm{Bi}$ and $\mathrm{Bi}^{\prime}$ cannot be absorbed in the terms in $\mathrm{Ai}$ and $\mathrm{Ai}^{\prime}$ because they are exponentially larger.

That (12.20) is valid in the wider range $t \geq-1+\delta$ can be proved as follows. In (8.11) replace $\mu$ by $\mu e^{\frac{3}{4} \pi i}$. Then we obtain

$$
\begin{aligned}
U\left(\frac{1}{2} i \mu^{2}, e^{\frac{3}{4} \pi i} \mu t \sqrt{ } 2\right) \sim 2 \pi^{\frac{1}{2}} e^{\frac{1}{4} \pi i} \mu^{\frac{1}{3}} g & \left(\mu e^{\frac{3}{4} \pi i}\right)\left(\frac{\zeta}{t^{2}-1}\right)^{\frac{1}{4}} \\
\times & {\left[\operatorname{Ai}\left(-\mu^{\frac{4}{3}} \zeta\right) \sum_{s=0}^{\infty}(-)^{s} \frac{A_{s}(\zeta)}{\mu^{4 s}}+\frac{\operatorname{Ai}^{\prime}\left(-\mu^{\frac{4}{3}} \zeta\right)}{\mu^{\frac{8}{3}}} \sum_{s=0}^{\infty}(-)^{s} \frac{B_{s}(\zeta)}{\mu^{4 s}}\right] }
\end{aligned}
$$

valid when $t \in \mathbf{T}\left(\frac{3}{4} \pi\right)$ and so including $t \geq-1+\delta$ (cf. fig. 7). Equation (12.1), with $t$ replaced by $-t$, shows that the left-hand side equals

$$
2^{-\frac{1}{2}} e^{-\frac{1}{8} \pi \mu^{2}} e^{-i\left(\frac{1}{2} \phi_{2}+\frac{1}{8} \pi\right)}\left[k^{-\frac{1}{2}} W\left(\frac{1}{2} \mu^{2},-\mu t \sqrt{ } 2\right)+i k^{\frac{1}{2}} W\left(\frac{1}{2} \mu^{2}, \mu t \sqrt{ } 2\right)\right] .
$$

Also from (6.1) and (12.7) we derive

$$
\begin{aligned}
g\left(\mu e^{\frac{3}{4} \pi i}\right) & \sim 2^{\frac{1}{4} i \mu^{2}-\frac{1}{4}} e^{\frac{1}{4} \mu^{2}} e^{-\frac{3}{8} \pi i+\frac{3}{8} \pi \mu^{2}} \mu^{-\frac{1}{2} i \mu^{2}-\frac{1}{2}}(1+i L)^{-1} \\
& \simeq e^{\frac{1}{2} \pi \mu^{2}-\frac{1}{2} \pi i} g\left(\mu e^{-\frac{1}{4} \pi i}\right) \\
& =2^{-\frac{1}{2}} e^{\frac{3}{8} \pi \mu^{2}} e^{-i\left(\frac{1}{2} \phi_{2}+\frac{3}{8} \pi\right)} l(\mu)
\end{aligned}
$$

(cf. (12.6) and (12.9)). The expansion (12.20) may now be obtained by substituting (12.22) and (12.23) in (12.21), equating real parts and using the first of (12.3).

(iii) a negative. When $a$ is negative the differential equation has no turning points on the real axis and we may confine our attention to the exponential-type expansions.

From (2.16), (2.17) and (2.18), we have

$k^{-\frac{1}{2}} W\left(-\frac{1}{2} \mu^{2}, \mu t \sqrt{ } 2\right)+i k^{\frac{1}{2}} W\left(-\frac{1}{2} \mu^{2},-\mu t \sqrt{ } 2\right)=2^{\frac{1}{2}} e^{-\frac{1}{8} \pi \mu^{2}} e^{i\left(\frac{1}{2} \phi_{2}+\frac{1}{8} \pi\right)} U\left(-\frac{1}{2} i \mu^{2}, e^{-\frac{1}{4} \pi i} \mu t \sqrt{ } 2\right)$,

where

$$
k=\left(1+e^{-\pi \mu^{2}}\right)^{\frac{1}{2}}-e^{-\frac{1}{2} \pi \mu^{2}}=1+O\left(e^{-\frac{1}{2} \pi \mu^{2}}\right),
$$


and

$$
e^{\frac{1}{2} i \phi_{2}}= \pm\left\{\Gamma\left(\frac{1}{2}-\frac{1}{2} i \mu^{2}\right) /\left|\Gamma\left(\frac{1}{2}-\frac{1}{2} i \mu^{2}\right)\right|\right\}^{\frac{1}{2}}=e^{\frac{1}{4} i \mu^{2}}\left(\frac{1}{2} \mu^{2}\right)^{-\frac{1}{4} i \mu^{2}}\left\{1+O\left(\mu^{-2}\right)\right\}
$$

(cf. (12.2) and (12.3)).

In (4.3) replace $\mu, t$ by $\mu e^{\frac{1}{4} \pi i}, t e^{-\frac{1}{2} \pi i}$, respectively. Then we obtain

$$
U\left(-\frac{1}{2} i \mu^{2}, e^{-\frac{1}{4} \pi i} \mu t \sqrt{ } 2\right) \sim g\left(\mu e^{\frac{1}{4} \pi i}\right) \frac{e^{-i \mu 2 \xi(-i t)}}{\left(-t^{2}-1\right)^{\frac{1}{4}}} \sum_{s=0}^{\infty}(-i)^{s} \frac{u_{s}(-i t)}{\left(-t^{2}-1\right)^{\frac{3}{2} s}} \frac{1}{\mu^{2 s}} .
$$

The region of validity of this expansion, $e^{\frac{1}{2} \pi i} \mathbf{S}\left(\frac{1}{4} \pi\right)$, includes the whole of the real $t$-axis; this is clear from figures 2 (b), 3 (iii) and 1.

Substituting (12.25), (12.26), and (12.27) in (12.24) in the manner of (i) and (ii) and determining the branches of the many-valued functions, we obtain finally

$$
\begin{aligned}
W\left(-\frac{1}{2} \mu^{2}, \mu t \sqrt{ } 2\right) \sim \frac{l(\mu)}{\left(t^{2}+1\right)^{\frac{1}{4}}}\left[\cos \left(\mu^{2} \bar{\xi}+\frac{1}{4} \pi\right) \sum_{s=0}^{\infty} \frac{(-)^{s} \bar{u}_{2 s}(t)}{\left(t^{2}+1\right)^{3 s} \mu^{4 s}}\right. & \\
& \left.\quad-\sin \left(\mu^{2} \bar{\xi}+\frac{1}{4} \pi\right) \sum_{s=0}^{\infty} \frac{(-)^{s} \bar{u}_{2 s+1}(t)}{\left(t^{2}+1\right)^{3 s+\frac{3}{2}} \mu^{4 s+2}}\right],
\end{aligned}
$$

uniformly when $-\infty<t<\infty$. Here $\bar{\xi} \equiv \bar{\xi}(t)$ and $\bar{u}_{s}(t)$ are given by (11.7), (11.9), and (11.11), and $l(\mu)$ is calculable from (12.11).

The corresponding expansion for the derivative is

$$
\begin{aligned}
W^{\prime}\left(-\frac{1}{2} \mu^{2}, \mu t \sqrt{ } 2\right) \sim-\frac{\mu}{\sqrt{ } 2} l(\mu)\left(t^{2}+1\right)^{\frac{1}{4}}\left[\sin \left(\mu^{2} \bar{\xi}+\frac{1}{4} \pi\right) \sum_{s=0}^{\infty} \frac{(-)^{s} \bar{v}_{2 s}(t)}{\left(t^{2}+1\right)^{3 s} \mu^{4 s}}\right. & \\
& \left.+\cos \left(\mu^{2} \bar{\xi}+\frac{1}{4} \pi\right) \sum_{s=0}^{\infty} \frac{(-)^{s} \bar{v}_{2 s+1}(t)}{\left(t^{2}+1\right)^{3 s+\frac{3}{2}} \mu^{4 s+2}}\right],
\end{aligned}
$$

$\bar{v}_{s}(t)$ being given by (11.13) and (11.14).

\section{Numerical Examples}

Here we illustrate the use of the Airy-type expansions by two examples. It may be remarked that applications would be greatly facilitated by the preparation of suitable numerical tables of the functions $g(\mu), l(\mu), \zeta(t),\left\{\zeta /\left(t^{2}-1\right)\right\}^{\frac{1}{4}}$ and $A_{s}(\zeta), B_{s}(\zeta), C_{s}(\zeta), D_{s}(\zeta)(s=0,1, \ldots)$. In the present examples these functions are calculated directly from formulas given in this paper.

It will appear from the examples that the early coefficients in the expansions decrease in magnitude as $s$ increases. This property, shared with uniform asymptotic expansions for Bessel functions of large order ([8], sec. 6), makes the expansions well suited to numerical work.

Example 1. To evaluate $W(a, \pm x), W^{\prime}(a, \pm x)$ for $a=8, x=7$.

We use the expansions (12.18), (12.19), and (12.20). Here

Hence

$$
\mu=4, \quad t=7 /(4 \sqrt{ } 2)=1.237436867 .
$$

and from (12.11), (12.12)

$$
2^{\frac{1}{2}} e^{\frac{1}{4} \pi \mu^{2}}=4.05527596 \times 10^{5},
$$

so that

$$
l(\mu) \sim\left(2^{\frac{1}{4}} / 2\right)(1-0.000003391-0.000000006+\ldots)=0.594601538,
$$

$$
\pi^{\frac{1}{2}} \mu^{\frac{1}{3}} l(\mu)=1.67296798 .
$$

Next, from (7.4) we have

$$
\zeta^{\frac{3}{2}}=\frac{3}{4} t\left(t^{2}-1\right)^{\frac{1}{2}}-\frac{3}{4} \ln \left\{t+\left(t^{2}-1\right)^{\frac{1}{2}}\right\}=0.169329581 .
$$


Hence

$$
\zeta=0.306070793, \quad \zeta^{\frac{1}{2}}=0.553236652, \quad\left\{\zeta /\left(t^{2}-1\right)\right\}^{\frac{1}{4}}=0.871225817 .
$$

From (4.8) and (4.11) we compute

$$
\mathscr{A}_{0}=1, \quad \mathscr{A}_{1}=-0.59504451, \quad \mathscr{A}_{2}=2.9247970, \quad \mathscr{A}_{3}=-26.482647,
$$

and from (8.13)

$$
\begin{array}{llll}
a_{0}=1, & a_{1}=\frac{5}{48}, & a_{2}=\frac{385}{4608}, & a_{3}=\frac{85085}{663552}, \\
b_{0}=1, & b_{1}=-\frac{7}{48}, & b_{2}=-\frac{455}{4608}, & b_{3}=-\frac{95095}{663552} .
\end{array}
$$

Substitution in (8.12) yields

$$
A_{0}(\zeta)=1, \quad A_{1}(\zeta)=-0.006490, \quad B_{0}(\zeta)=-0.0363797, \quad B_{1}(\zeta)=0.01202
$$

Next, we have $\mu^{\frac{4}{3}} \zeta=1.94342840$, and by interpolation in table VII of [3]

$$
\begin{aligned}
& \operatorname{Bi}\left(-\mu^{\frac{4}{3}} \zeta\right)=-0.39524090, \quad \operatorname{Bi}^{\prime}\left(-\mu^{\frac{4}{3}} \zeta\right)=0.32386134 \\
& \mathrm{Ai}\left(-\mu^{\frac{4}{3}} \zeta\right)=0.26162602, \quad \mathrm{Ai}^{\prime}\left(-\mu^{\frac{4}{3}} \zeta\right)=0.59097966 .
\end{aligned}
$$

The content of the square brackets on the right of (12.18) is accordingly

$-0.39524090(1+0.00002535+\ldots)+4^{-\frac{8}{3}} \times 0.32386134(-0.0363797-0.0000470+\ldots)$

Substituting this value and (13.1), (13.2), (13.3) in (12.18), we obtain

Similarly,

$$
W(8,7)=-\frac{1.67296798 \times 0.871225817 \times 0.39554353}{4.05527596 \times 10^{5}}=\mathbf{- 0 . 1 4 2 1 6 4 8 5} \times \mathbf{1 0}^{-5} .
$$

$W(8,-7)=1.67296798 \times 0.871225817 \times 0.26109871 \times 4.05527596 \times 10^{5}=\mathbf{1 . 5 4 3 2 7} \mathbf{5 7} \times \mathbf{1 0}^{5}$.

For the derivatives, we have first

$$
2^{-\frac{1}{2}} \pi^{\frac{1}{2}} \mu^{\frac{2}{3}} l(\mu)=1.87784306 .
$$

From (4.14) and (4.16), we compute

$$
\mathscr{B}_{0}=1, \quad \mathscr{B}_{1}=1.00283725, \quad \mathscr{B}_{2}=-3.5232659, \quad \mathscr{B}_{3}=29.919423,
$$

and thence from (8.19)

$$
C_{0}(\zeta)=-0.0783370, \quad C_{1}(\zeta)=0.00971, \quad D_{0}(\zeta)=1, \quad D_{1}(\zeta)=0.007604 .
$$

Hence

$$
\begin{aligned}
& W^{\prime}(8,7)=-\frac{1.87784306 \times 0.31897315}{4.05527596 \times 10^{5} \times 0.871225817}=\mathbf{- 0 . 1 6 9 5 3 ~ 6 1 3} \times \mathbf{1 0}^{-5}, \\
& W^{\prime}(8,-7)=\frac{1.87784306 \times 0.59419143 \times 4.05527596 \times 10^{5}}{0.871225817}=\mathbf{5 . 1 9 3 6 8 2 0} \times \mathbf{1 0}^{5} .
\end{aligned}
$$

The results of this example agree adequately with the entries given in [4], table I. 
Example 2. To evaluate the Hermite polynomial $H_{n}(x)$ for $n=10, x=4$ by means of the expansion (8.11).

Here (cf. (11.23))

$$
\mu=\sqrt{ } 21=4.58257569, \quad t=4 / \sqrt{ } 21=0.872871561 .
$$

From (6.1) and (4.12)

$$
1 / g(\mu) \sim 2^{5.5} e^{5.25}(21)^{-5}(1+0.001984127-0.000001052+\ldots)=2.11580149 \times 10^{-3},
$$

and hence

Next, from (11.21)

$$
2 \pi^{\frac{1}{2}} \mu^{\frac{1}{3}} g(\mu)=2.78291471 \times 10^{3} .
$$

$$
(-\zeta)^{\frac{3}{2}}=\frac{3}{4} \cos ^{-1} t-\frac{3}{4} t\left(1-t^{2}\right)^{\frac{1}{2}}=0.062866477
$$

Hence

$$
\zeta=-0.158105174, \quad(-\zeta)^{\frac{1}{2}}=0.397624413, \quad\left\{\zeta /\left(t^{2}-1\right)\right\}^{\frac{1}{4}}=0.90271123 .
$$

When $\zeta$ is negative, real forms of equations (8.12) and (4.8) are

$$
A_{s}(\zeta)=(-)^{s} \sum_{m=0}^{2 s} b_{m}(-\zeta)^{-\frac{3}{2} m} \overline{\mathscr{A}}_{2 s-m}, \quad(-\zeta)^{\frac{1}{2}} B_{s}(\zeta)=(-)^{s-1} \sum_{m=0}^{2 s+1} a_{m}(-\zeta)^{-\frac{3}{2} m} \overline{\mathscr{A}}_{2 s-m+1}
$$

where

$$
\overline{\mathscr{A}}_{s}=u_{s}(t) /\left(1-t^{2}\right)^{\frac{3}{2} s} \text {. }
$$

In the present example we find

and thence

$$
\overline{\mathscr{A}_{0}}=1, \quad \overline{\mathscr{A}}_{1}=-1.63978319, \quad \overline{\mathscr{A}}_{2}=21.1903125, \quad \overline{\mathscr{A}}_{3}=-516.52363,
$$

$$
A_{0}(\zeta)=1, \quad A_{1}(\zeta)=-0.010243, \quad B_{0}(\zeta)=-0.0431757, \quad B_{1}(\zeta)=0.0166
$$

Next, $\mu^{\frac{4}{3}} \zeta=-1.20344324$. Interpolation in table I of [3], yields

$$
\operatorname{Ai}\left(\mu^{\frac{4}{3}} \zeta\right)=0.52582209, \quad \operatorname{Ai}^{\prime}\left(\mu^{\frac{4}{3}} \zeta\right)=0.10920810 .
$$

Substituting these results in (8.11), we obtain

$$
U\left(-\frac{21}{2}, 4 \sqrt{ } 2\right)=2.78291471 \times 10^{3} \times 0.90271123 \times 0.52572856=1.32071865 \times 10^{3},
$$

and thence using (11.23)

$$
H_{10}(4)=2^{5} e^{8} U\left(-\frac{21}{2}, 4 \sqrt{ } 2\right)=\mathbf{1 . 2 5 9 8 4 2 2} \times 10^{8} .
$$

The exact value of this polynomial, computed from explicit expressions given on page 75 of [4], is 125984224 .

\section{Part 5. Zeros and Associated Values}

14. Zeros of $U(a, z), U^{\prime}(a, z), \bar{U}(a, z)$, and $\bar{U}^{\prime}(a, z)$

(i) a negative; $-2(1-\delta) \sqrt{ }(-a) \leq z \leq 2 \sqrt{ }(-a)$. Let the $s$ th real zeros, counted in descending order away from the point $z=2 \sqrt{ }(-a)$, of the functions $U(a, z), U^{\prime}(a, z), \bar{U}(a, z)$, and $\bar{U}^{\prime}(a, z)$ be denoted by $u_{a, s}, u_{a, s}^{\prime}, \bar{u}_{a, s}$, and $\bar{u}_{a, s}^{\prime}$, respectively.

The expansion (8.11) is of exactly the same form as the expansion (4.24) of [8]. The analysis of section 7 of that paper is therefore immediately applicable, and we find

$$
u_{a, s} \sim 2^{\frac{1}{2}} \mu\left[p_{0}(\alpha)+\frac{p_{1}(\alpha)}{\mu^{4}}+\frac{p_{2}(\alpha)}{\mu^{8}}+\ldots\right]
$$


as $\mu \equiv \sqrt{ }(-2 a) \rightarrow+\infty, s$ fixed. Here

$$
\alpha=\mu^{-\frac{4}{3}} a_{s},
$$

$a_{s}$ denoting the sth negative zero of the function $\mathrm{Ai}$ and not to be confused with the $a_{m}$ of (8.13). The coefficients $p_{\tau}(\zeta)$ are given by

$$
p_{0}(\zeta)=t(\zeta), \quad p_{1}(\zeta)=\alpha_{1}(\zeta) t^{\prime}(\zeta), \quad p_{2}(\zeta)=\alpha_{2}(\zeta) t^{\prime}(\zeta)+\frac{1}{2}\left\{\alpha_{1}(\zeta)\right\}^{2} t^{\prime \prime}(\zeta), \ldots
$$

where $t(\zeta)$ is the function inverse to $\zeta(t)$, defined by (11.21), and the $\alpha_{r}(\zeta)$ are given by relations of the form of (7.13) of [8]. Explicitly,

$$
p_{1}(\zeta)=\frac{t^{3}-6 t}{24\left(t^{2}-1\right)^{2}}+\frac{5}{48\left\{\left(t^{2}-1\right) \zeta^{3}\right\}^{\frac{1}{2}}} .
$$

The corresponding expansion for the associated value $U^{\prime}\left(a, u_{a, s}\right)$ can be found by substituting (14.1) in (8.15). More convenient expressions for the coefficients are obtained however, if we substitute instead in the equation

$$
U^{\prime}\left(a, u_{a, s}\right)= \pm(2 \pi)^{\frac{1}{4}}\left\{\Gamma\left(\frac{1}{2}-a\right)\right\}^{\frac{1}{2}}\left(-\frac{d u_{a, s}}{d s}\right)^{-\frac{1}{2}}
$$

obtained from eq (2.15) of [6] and the second of (2.13) in the present paper. This yields the required result in the form

where

$$
U^{\prime}\left(a, u_{a, s}\right) \sim \pi^{\frac{1}{4}} \mu^{\frac{1}{6}}\left\{\Gamma\left(\frac{1}{2}+\frac{1}{2} \mu^{2}\right)\right\}^{\frac{1}{2}} \mathrm{Ai}^{\prime}\left(a_{s}\right) \psi(\alpha)\left[1+\sum_{r=1}^{\infty} \frac{P_{r}(\alpha)}{\mu^{4 r}}\right],
$$

$$
\psi(\zeta) \equiv \frac{1}{\phi(\zeta)}=\left(\frac{t^{2}-1}{\zeta}\right)^{\frac{1}{4}}
$$

and the coefficients $P_{r}(\zeta)$ satisfy the asymptotic identity

Thus

$$
\left[1+\sum_{r=1}^{\infty} \frac{P_{r}(\zeta)}{\mu^{4 r}}\right]^{-2} \sim 1+\{\psi(\zeta)\}^{2} \sum_{r=1}^{\infty} \frac{p_{r}^{\prime}(\zeta)}{\mu^{4 r}}
$$

Explicitly,

$$
P_{1}(\zeta)=-\frac{1}{2}\{\psi(\zeta)\}^{2} p_{1}^{\prime}(\zeta), \quad P_{2}(\zeta)=\frac{3}{2}\left\{P_{1}(\zeta)\right\}^{2}-\frac{1}{2}\{\psi(\zeta)\}^{2} p_{2}^{\prime}(\zeta)
$$

$$
P_{1}(\zeta)=\frac{t^{4}-15 t^{2}-6}{48\left(t^{2}-1\right)^{3}}+\frac{5 t}{96\left(t^{2}-1\right)^{\frac{3}{2}} \zeta^{\frac{3}{2}}}+\frac{5}{64 \zeta^{3}} .
$$

For the zeros of the derivatives of the parabolic cylinder functions, we may show by similar analysis that

where

$$
u_{a, s}^{\prime} \sim 2^{\frac{1}{2}} \mu\left[q_{0}(\beta)+\frac{q_{1}(\beta)}{\mu^{4}}+\frac{q_{2}(\beta)}{\mu^{8}}+\ldots\right]
$$

$$
\beta=\mu^{-\frac{4}{3}} a_{s}^{\prime}
$$

$$
q_{0}(\zeta)=t(\zeta), \quad q_{1}(\zeta)=\beta_{1}(\zeta) t^{\prime}(\zeta), \quad q_{2}(\zeta)=\beta_{2}(\zeta) t^{\prime}(\zeta)+\frac{1}{2}\left\{\beta_{1}(\zeta)\right\}^{2} t^{\prime \prime}(\zeta), \ldots
$$

and the $\beta_{r}(\zeta)$ are given by eq $(7.22)$ of [8].

The equation corresponding to (14.5) is

$$
U\left(a, u_{a_{\mathbf{v}} s}^{\prime}\right)= \pm(2 \pi)^{\frac{1}{4}}\left\{\Gamma\left(\frac{1}{2}-a\right)\right\}^{\frac{1}{2}}\left(-a-\frac{1}{4} u_{a, s}^{\prime 2}\right)^{-\frac{1}{2}}\left(-\frac{d u_{a, s}^{\prime}}{d s}\right)^{-\frac{1}{2}}
$$


and from it we derive

$$
U\left(a, u_{a, s}^{\prime}\right) \sim \frac{(4 \pi)^{\frac{1}{4}}}{\mu^{\frac{1}{6}}}\left\{\Gamma\left(\frac{1}{2}+\frac{1}{2} \mu^{2}\right)\right\}^{\frac{1}{2}} \operatorname{Ai}\left(a_{s}^{\prime}\right) \phi(\beta)\left[1+\sum_{r=1}^{\infty} \frac{Q_{r}(\beta)}{\mu^{4 r}}\right]
$$

in which the coefficients satisfy the identity

$$
\left[1+\sum_{r=1}^{\infty} \frac{Q_{r}(\zeta)}{\mu^{4 r}}\right]^{-2} \sim \frac{\{\phi(\zeta)\}^{2}}{\zeta}\left[\left\{\sum_{r=0}^{\infty} \frac{q_{r}(\zeta)}{\mu^{4 r}}\right\}^{2}-1\right]\left[\sum_{r=0}^{\infty} \frac{q_{r}^{\prime}(\zeta)}{\mu^{4 r}}\right]
$$

For the function $\bar{U}(a, z)$, the expansions corresponding to (14.1), (14.6), (14.11), and (14.15) are obtained on replacing the symbols $U, u, \mathrm{Ai}$, and $a_{s}$ by $\bar{U}, \bar{u}, \mathrm{Bi}$, and $b_{s}$, respectively.

(ii) a negative; other zeros. Since the zeros of the function Ai are all real and negative, it follows from (8.11) that for all sufficiently large positive values of the parameter $\mu$, the zeros of $U\left(-\frac{1}{2} \mu^{2}, \mu t \sqrt{ } 2\right)$ in the $t$-domain $\mathbf{T}(0)$ (the unshaded region of fig. $7($ a)), all lie in the interval $-1<t<1$. This is also true of the zeros of the derived function $U^{\prime}\left(-\frac{1}{2} \mu^{2}, \mu t \sqrt{ } 2\right)$. The function $\bar{U}\left(-\frac{1}{2} \mu^{2}, \mu t \sqrt{ } 2\right)$ and its derivative however, have complex zeros in this region corresponding to the complex zeros of $\mathrm{Bi}$ and $\mathrm{Bi}^{\prime}$ (cf. [8], appendix).

In the complementary region $C \mathbf{T}(0)$ the distribution of the zeros of $U\left(-\frac{1}{2} \mu^{2}, \mu t \sqrt{ } 2\right)$ can be investigated by reversion of the expansion (9.7). The situation is more complicated; the pattern of the zeros depends largely upon the non-integer part of $\frac{1}{2} \mu^{2}$. It should be noted that expansions of zeros in the interval $-1<t<1$ of fixed enumeration, counted away from $t=-1$, come within this category.

(iii) a complex. The extension of the results of this section to general values of $a$ presents no difficulty. We readily see, for example, that when $\epsilon \leq \arg \mu \leq \pi-\epsilon$ the zeros of $U\left(-\frac{1}{2} \mu^{2}, \mu t \sqrt{ } 2\right)$ in the $t$-domain $\mathbf{T}(\arg \mu)$ are asymptotically close to the principal curve AQ, illustrated in figure 4 .

\section{Zeros of $W(a, x)$ and $W^{\prime}(a, x)$}

We denote the sth positive zeros of the functions $W(a, x), W^{\prime}(a, x), W(a,-x)$ and $W^{\prime}(a,-x)$, by $w_{a, s}, w_{a, s}^{\prime}, \bar{w}_{a, s}$ and $\bar{w}_{a, s}^{\prime}$, respectively.

(i) a positive. Writing $\mu=\sqrt{ }(2 a)$ and

$$
\alpha=\mu^{-\frac{4}{3}} b_{s}, \quad \beta=\mu^{-\frac{4}{3}} b_{s}^{\prime},
$$

where $b_{s}$ and $b_{s}^{\prime}$ are the $s$ th negative zeros of $\mathrm{Bi}$ and $\mathrm{Bi}^{\prime}$ respectively, we find by reversion of (12.18) and (12.20)

$$
\begin{aligned}
& w_{a . s} \sim 2^{\frac{1}{2}} \mu\left[p_{0}(-\alpha)-\frac{p_{1}(-\alpha)}{\mu^{4}}+\frac{p_{2}(-\alpha)}{\mu^{8}}-\ldots\right], \\
& 2^{\frac{1}{2}} e^{\frac{1}{2} \pi a} W^{\prime}\left(a, w_{a, s}\right) \sim-2^{-\frac{1}{4}} \pi^{\frac{1}{2}} \mu^{\frac{1}{6}} \mathrm{Bi}^{\prime}\left(b_{s}\right) \psi(-\alpha)\left[1+\sum_{r=1}^{\infty}(-)^{r} \frac{P_{r}(-\alpha)}{\mu^{4 r}}\right], \\
& w^{\prime}{ }_{a, s} \sim 2^{\frac{1}{2}} \mu\left[q_{0}(-\beta)-\frac{q_{1}(-\beta)}{\mu^{4}}+\frac{q_{2}(-\beta)}{\mu^{8}}-\ldots\right], \\
& 2^{\frac{1}{2}} e^{\frac{1}{2} \pi a} W\left(a, w_{a, s}^{\prime}\right) \sim 2^{\frac{1}{4}} \pi^{\frac{1}{2}} \mu^{-\frac{1}{6}} \mathrm{Bi}\left(b_{s}^{\prime}\right) \phi(-\beta)\left[1+\sum_{r=1}^{\infty}(-)^{r} \frac{Q_{r}(-\beta)}{\mu^{4 r}}\right],
\end{aligned}
$$

as $a \rightarrow+\infty$, uniformly with respect to $s$. Here $\psi(\zeta), \phi(\zeta), p_{r}(\zeta), P_{r}(\zeta), q_{r}(\zeta)$ and $Q_{r}(\zeta)$ are the functions defined in section 14(i).

With $\alpha, \beta$ given by (14.2) and (14.12) instead of (15.1) and $\mathrm{Bi}, b$ replaced by $\mathrm{Ai}, a$, the right-hand sides of (15.2), (15.3), (15.4), and (15.5) are the corresponding expansions of $\bar{w}_{a, s}$, $-2^{-\frac{1}{2}} e^{-\frac{1}{2} \pi a} W^{\prime}\left(a,-\bar{w}_{a, s}\right), \bar{w}_{a, s}^{\prime}$ and $2^{-\frac{1}{2}} e^{-\frac{1}{2} \pi a} W\left(a,-\bar{w}_{a, s}^{\prime}\right)$, respectively. 
(ii) a negative. Let $\mu=\sqrt{ }(-2 a)$, and

$$
t=\tau(\bar{\xi})
$$

be the function inverse to $\bar{\xi}(t)$, defined by (11.7). Then by reversion of the expansion (12.28), we find

$$
w_{a, s} \sim 2^{\frac{1}{2}} \mu\left[\tau+\frac{\mathrm{e}_{1}(\tau)}{\mu^{4}}+\frac{\mathrm{e}_{2}(\tau)}{\mu^{8}}+\cdots\right]
$$

as $a \rightarrow-\infty$, uniformly with respect to $s$. Here

$$
\tau=\tau\left\{\frac{\left(s-\frac{3}{4}\right) \pi}{\mu^{2}}\right\},
$$

and the leading coefficients are given by the formulas

$$
\mathrm{e}_{1}(\tau)=\frac{\tau^{3}+6 \tau}{24\left(\tau^{2}+1\right)^{2}}, \quad \mathrm{e}_{2}(\tau)=\frac{56 \tau^{9}+247 \tau^{7}+291 \tau^{5}-2520 \tau^{3}+3780 \tau}{5760\left(\tau^{2}+1\right)^{5}} .
$$

The expansion of the associated value of the derivative can be obtained by substitution of (15.7) in the equation

$$
W^{\prime}\left(a, w_{a, s}\right)= \pm \pi^{\frac{1}{2}}\left(\frac{d w_{a . s}}{d s}\right)^{-\frac{1}{2}}
$$

obtained from eq (2.15) of [6] and (2.19). Thence we derive

where

$$
W^{\prime}\left(a, w_{a, s}\right) \sim(-)^{s} 2^{-\frac{1}{4}} \mu^{\frac{1}{2}}\left(\tau^{2}+1\right)^{\frac{1}{4}}\left[1+\sum_{r=1}^{\infty} \frac{E_{r}(\tau)}{\mu^{4 r}}\right],
$$

$$
E_{1}(\tau)=\frac{\tau^{4}+15 \tau^{2}-6}{48\left(\tau^{2}+1\right)^{3}}, \quad E_{2}(\tau)=\frac{112 \tau^{10}+489 \tau^{8}-98 \tau^{6}-34995 \tau^{4}+80460 \tau^{2}-7020}{23040\left(\tau^{2}+1\right)^{6}} .
$$

In a similar manner we obtain from (12.29)

where now

$$
w_{a, s}^{\prime} \sim 2^{\frac{1}{2}} \mu\left[\tau+\frac{f_{1}(\tau)}{\mu^{4}}+\frac{f_{2}(\tau)}{\mu^{8}}+\ldots\right]
$$

$$
\tau=\tau\left\{\frac{\left(s-\frac{1}{4}\right) \pi}{\mu^{2}}\right\}
$$

and

$$
f_{1}(\tau)=\frac{\tau^{3}-6 \tau}{24\left(\tau^{2}+1\right)^{2}}, \quad f_{2}(\tau)=\frac{56 \tau^{9}+247 \tau^{7}+651 \tau^{5}+2640 \tau^{3}-3420 \tau}{5760\left(\tau^{2}+1\right)^{5}} .
$$

Finally, from

we find

$$
W\left(a, w_{a, s}^{\prime}\right)= \pm \pi^{\frac{1}{2}}\left(\frac{1}{4} w_{a, s}^{\prime 2}-a\right)^{-\frac{1}{2}}\left(\frac{d w_{a, s}^{\prime}}{d s}\right)^{-\frac{1}{2}}
$$

$$
W\left(a, w_{a, s}^{\prime}\right) \sim(-)^{s} 2^{\frac{1}{4}} \mu^{-\frac{1}{2}}\left(\tau^{2}+1\right)^{-\frac{1}{4}}\left[1+\sum_{r=1}^{\infty} \frac{F_{r}(\tau)}{\mu^{4 \tau}}\right]
$$

where $\tau$ is again given by (15.14), and

$$
F_{1}(\tau)=\frac{-\tau^{4}-9 \tau^{2}+6}{48\left(\tau^{2}+1\right)^{3}}, \quad F_{2}(\tau)=\frac{-112 \tau^{10}-479 \tau^{8}-142 \tau^{6}+25725 \tau^{4}-67500 \tau^{2}+7380}{23040\left(\tau^{2}+1\right)^{6}} .
$$

If in the right-hand sides of (15.7) and (15.11) we take the value (15.14) for $\tau$ instead of (15.8), we obtain the asymptotic expansions of $\bar{w}_{a, s}$ and $-W^{\prime}\left(a,-\bar{w}_{a, s}\right)$, respectively. Similarly the right-hand sides of (15.13) and (15.17) with $\tau$ given by (15.8) are the expansions of $\bar{w}_{a, s}^{\prime}$ and $-W\left(a,-\bar{w}_{a, s}^{\prime}\right)$. 
It may be noticed that some of the numerical coefficients occurring in the expressions (14.4) and (14.10) for the coefficients in the reverted Airy-type expansions are the same as those in the expressions (15.9) and (15.12) pertaining to the reverted exponential-type expansions. This is no mere coincidence; we may expect that by suitable re-expansion of the reverted Airy forms, and subsequent comparison with exponential-type expansions obtained by reversion of (5.11), we shall obtain explicit expressions for the coefficients $p_{r}$ and $P_{r}$ in terms of the $e_{\tau}$ and $E_{r}$ respectively, analogous to the formulas (8.12). Similarly for $q_{r}$ and $Q_{r}$.

\section{Numerical Examples}

Example 1. To compute the smallest positive zero of $W(10, x)$ and the corresponding $W^{\prime}(10, x)$.

We use (15.2) and (15.3) with $\mu=\sqrt{ } 20=4.47213595, \quad s=1$. Entering table $\mathrm{V}$ of [3], we find

Hence

$$
b_{1}=-1.17371322, \quad \operatorname{Bi}^{\prime}\left(b_{1}\right)=0.60195789 .
$$

The value of $t$ is given by

$$
\zeta=-\alpha=-\mu^{-\frac{4}{3}} b_{1}=0.159297392 .
$$

$$
t\left(t^{2}-1\right)^{\frac{1}{2}}-\ln \left\{t+\left(t^{2}-1\right)^{\frac{1}{2}}\right\}=\frac{4}{3} \zeta^{\frac{3}{2}}=0.084771864 .
$$

Solving this equation by successive approximation, using Newton's rule, we find

and thence from (14.4)

$$
t=1.124896229=p_{0}(-\alpha),
$$

Hence

$$
p_{1}(-\alpha)=0.0296004 \text {. }
$$

$$
w_{10,1} \sim 2^{\frac{1}{2}} \times 4.47213595(1.124896229-0.000074001+\ldots)=\mathbf{7 . 1 1 4 0 0} 040 .
$$

For the derivative, we compute from (14.7) and (14.10)

$$
1 / \psi(-\alpha)=\phi(-\alpha)=0.880198053, \quad P_{1}(-\alpha)=0.009422 .
$$

Substitution in (15.3) then yields

$$
W^{\prime}\left(10, w_{10,1}\right) \sim-\frac{2^{-\frac{3}{4}} \pi^{\frac{1}{2}}(20)^{\frac{1}{12}} \times 0.60195789}{e^{5 \pi} \times 0.880198053}(1-0.00002356+\ldots)=-\mathbf{1 . 3 9 4 1 6} 8 \mathbf{6 5} \times \mathbf{1 0}^{-7} \text {. }
$$

By inverse and direct interpolation in table III of [4], we find

$$
w_{10,1}=7.11400000, \quad W^{\prime}\left(10, w_{10.1}\right)=-1.3941686 \times 10^{-7} .
$$

The discrepancy in the values of $w_{10,1}$ is undoubtedly due to neglect of the third term of the asymptotic series.

Example 2. To compute the fifth positive zero of $W^{\prime}(-10, x)$ and the corresponding $W(-10, x)$.

We use (15.13) and (15.17). From (15.14), (15.6), and (11.7) we see that the appropriate value of $\tau$ satisfies

$$
\tau\left(\tau^{2}+1\right)^{\frac{1}{2}}+\ln \left\{\tau+\left(\tau^{2}+1\right)^{\frac{1}{2}}\right\}=2 \bar{\xi}=2\left(5-\frac{1}{4}\right) \pi / 20=1.492256510 .
$$

Solving by successive approximation, we find

$$
\tau=0.693900720, \quad\left(\tau^{2}+1\right)^{\frac{1}{2}}=1.217168110,
$$


and thence from (15.15)

Thus

$$
f_{1}(\tau)=-0.07269508, \quad f_{2}(\tau)=-0.03321
$$

$$
w_{-10,5}^{\prime} \sim 2^{\frac{1}{2}} \times 4.47213595(0.693900720-0.000181738-0.000000208+\ldots)=\mathbf{4 . 3 8 7 4 6 2 7 6 .}
$$

From (15.18) we compute

and thence

$$
F_{1}(\tau)=0.00919198, \quad F_{2}(\tau)=-0.07882,
$$

$$
\begin{aligned}
W\left(-10, w_{-10,5}^{\prime}\right) \sim & -2^{\frac{1}{4}}(20)^{-\frac{1}{4}}(1.217168110)^{-\frac{1}{2}}(1+0.000022980-0.000000493+\ldots . .) \\
& =-\mathbf{0 . 5 0 9 7 2} \mathbf{3 2 9 7} .
\end{aligned}
$$

The results of this example agree with values obtained by interpolation in table III of [4].

\section{References}

[1] C. G. Darwin, On Weber's function, Quart. J. Mech. Appl. Math. 2, 311 (1949).

[2] A. Erdélyi, M. Kennedy, and J. L. McGregor, Parabolic cylinder functions of large order, J. Rat. Mech. Anal. 3, 459 (1954)

[3] J. C. P. Miller, The Airy integral, British Assoc. Math. Tables, Part-Volume B (Cambridge University Press, Cambridge, Eng. 1946).

[4] J. C. P. Miller, Tables of Weber parabolic cylinder functions, London: H. M. Stationery Office (1955).

[5] L. M. Milne-Thomson, The calculus of finite differences, London: Macmillan (1933).

[6] F. W. J. Olver, A new method for the evaluation of zeros of Bessel functions and of other solutions of second-order differential equations, Proc. Cambridge Philos. Soc. 46, 570 (1950).

[7] F. W. J. Olver, The asymptotic solution of linear differential equations of the second order for large values of a parameter, Phil. Trans. Roy. Soc. London, Ser. A $\mathbf{2 4}$, 307 (1954).

[8] F. W. J. Olver, The asymptotic expansion of Bessel functions of large order, Phil. Trans. Roy. Soc. London, Ser. A $\mathbf{2 4 7}, 328$ (1954).

[9] F. W. J. Olver, Uniform asymptotic expansions of solutions of linear second-order differential equations for large values of a parameter, Phil. Trans. Roy. Soc. London, Ser. A 250, 479 (1958).

[10] N. Schwid, The asymptotic forms of the Hermite and Weber functions, Trans. Amer. Math. Soc. 37, 339 (1935)

[11] G. N. Watson, A theory of asymptotic series, Phil. Trans. Roy. Soc. London, Ser. A 211, 279 (1911).

[12] G. N. Watson, The harmonic functions associated with the parabolic cylinder, Proc. London Math. Soc. 17, 116 (1918).

[13] E. T. Whittaker, On the functions associated with the parabolic cylinder in harmonic analysis, Proc. London Math. Soc. 35, 417 (1903).

[14] E. T. Whittaker and G. N. Watson, A course of modern analysis (Cambridge University Press, Cambridge, Eng., 1952).

[15] N. D. Kazarinoff, Asymptotic theory of second order differential equations with two simple turning points, Arch. Rat. Mech. Anal. 2, 129 (1958).

Washington, D. C.

(Paper 63B2-14) 

(including papers in outside journals)

\section{Selected Abstracts}

Diffraction of electromagnetic waves by smooth obstacles for grazing angles, J. R. Wait and A. M. Conda, J. Research NBS 63D, No. 2, 18 (1959).

The diffraction of electromagnetic waves by a convex cylindrical surface is considered. Attention is confined primarily to the region near the light-shadow boundary. The complexintegral representation for the field is utilized to obtain a correction to the Kirchoff theory. Numerical results are presented which illustrate the influence of surface curvature and polarization on the diffraction pattern. Good agreement with the experimental results of Bachynski and Neugebauer is obtained. The effect of finite conductivity is also considered.

Path antenna gain in an exponential atmosphere, W. J. Hartman and R. E. Wilkerson, J. Research NBS 63D, No. 3, 273 (1959).

The problem of determining path antenna gain is treated here in greater detail than previously. The method used here takes into account for the first time the exponential decrease of the gradient of refractive index with height, and a scattering cross section inversely proportional to the fifth power of the scattering angle. Results are given for all combinations of beamwidths and path geometry, assuming that symmetrical beams are used on both ends of the path and that atmospheric turbulence is isotropic. The result appears as a function of both of the beamwidths, in addition to other parameters, and thus the loss in gain cannot be determined independently for the transmitting and receiving antennas. The values of the loss in gain are generally lower than the previous estımates for which a comparison is possible.

Pattern synthesis for slotted-cylinder antennas, J. R. Wait and James Householder, J. Research NBS 63D, No. 3, 303 (1959).

The radiation from a cylinder excited by an array of axial slots is discussed. A procedure for synthesizing a given radiation pattern is developed with particular attention being paid to a Tchebyscheff type pattern. Specifying the side lobe level and the width of the main beam, the required source distributions are computed for a number of cases. The effect of using a finite number of slot elements to approximate the continuous source distribution is also considered.

Convexity of the field of a linear transformation, A. J. Goldman and M. Marcus, Can. Math. Bul. 2, No. 1, 15 (1959).

The field $F(A)$ of a linear transformation $A$ of unitary $n$-space $U_{n}$ is the set of complex numbers $z=(A x, x)$, where $x$ ranges over the unit sphere in $U_{n}$. The convexity of $F(A)$ (as a subset of the $z$-plane) is proved by a simple inductive argument which reduces the essential computations to the case $n=2$.

Graphical diagnosis of interlaboratory test results, W. J. Youden, Ind. Qual. Control XV, No. 11, 1 (1959).

This analysis of interlaboratory test results depends upon the availability from a number of laboratories of a single test result for each of two materials. The two results from each laboratory are used to plot a point using the $x$-axis for one material and the $y$-axis for the other. The resulting configuration of the points from the several laboratories permits deductions on the prevalence and extent of laboratory bias, the presence of sampling variation and the occurrence of blunders. In addition the graph provides an estimate of the precision of the test procedure results.
The calculation of the field in a homogeneous conductor with a wavy interface, J. R. Wait, Proc. IRE 47, 1155 (1959).

The field at any depth in a homogeneous conductor with a wavy interface is calculated. For purposes of illustration, the field above the interface is taken to be a uniform plane wave traveling in the horizontal direction.

Lower bounds for eigenvalues with application to the helium atom, N. W. Bazley, Proc. Nat. Acad. Sci. U.S. 45, No. 6, 850 (1959).

Let $A$ be a self-adjoint operator with domain $D$ in a Hilbert space. Suppose $A=A^{\prime}+\bar{A}$ where $\bar{A}$ is self-adjoint and $A^{\prime}$ is positive definite. The eigenvalue problem for $\bar{A}$, whose solution we assume known, gives rough lower bounds. If $\bar{u}_{i}$ $(i=1, \ldots, k)$ are $k$ discrete eigenvectors of $\bar{A}$ and if $p_{i}=\left(a^{\prime}\right)^{-1}$ $\bar{u}_{i}(i=1, \ldots, k)$ exist then one can substantially improve the lower bounds. The theory is applied to helium atom operator.

List of Titles *

Journal of Research, Section A. Physics and Chemistry, Volume 63A, No. 2, SeptemberOctober 1959. 70 cents.

Reflection of fast neutrons from water. Martin $J$. Berger and John W. Cooper.

Some vibrational-rotational bands of deuterated methanes. Harry C. Allen, Jr., and Earle K. Plyler.

Precise coulometric titration of acids and bases. John K. Taylor and Stanley W. Smith.

Heat of formation of titanium tetraiodide. W. H. Johnson, A. A. Gilliland, and E. J. Prosen.

Reactions of pentafluorohalobenzenes. Walter J. Pummer and Leo A. Wall.

Tritium-labeled compounds I. Radioassay of tritium-labeled compounds in "infinitely thick" films with a windowless, gas-flow, proportional contour. Horace S. Isbell, Harriet L. Frush, and Ruth Peterson.

Tritium-labeled compounds II. General-purpose apparatus, and procedures for the preparation, analysis, and use of tritium oxide and tritiumlabeled lithium borohydride. Horace S. Isbell and Joseph D. Moyer.

Preparation of trichloride and tetrachloride of molybdenum. Dwight E. Couch and Abner Brenner. Infrared spectra of asphalts. Burton D. Beitchman.

Journal of Research, Section C. Engineering and Instrumentation, Volume 63C, No. 2, OctoberDecember 1959.75 cents.

Conical coaxial capacitors and their advantages. M. C. Selby.

A photoelectric followup and recording system, and its application to remote observations of the beam in high precision balances. H. A. Bowman and L. B. Macurdy. 
A stroboscopic vibration analyzer. S. Edelman, R. Brooks, S. Saito, E. Jones, and E. R. Smith.

Evaluation of lens distortion by the inverse nodal slide. Francis H. Washer and Walter R. Darling.

Evaluation of lens distortion by the modified goniometric method. Francis E. Washer and Walter R. Darling.

Proposed criteria for defining load failure of beams, floors, and roof constructions during fire tests. J. V. Ryan and A. F. Robertson.

Conductive flooring for hospital operating rooms. Thomas H. Boone, Francis L. Hermach, Edgar H. MacArthur, and Rita C. McAuliff.

Measurement of the aging of rubber vulcanizates. J. Mandel, F. L. Roth, M. N. Steel, and R. D. Stiehler.

Journal of Research, Section D. Radio Propagation, Volume 63D, No. 2, September-October 1959. 70 cents.

Stratification in the lower ionosphere. C. Ellyett and J. M. Watts.

Effect of small irregularities on the constitutive relations for the ionosphere. K. G. Budden.

Ionospheric investigations using the sweep-frequency pulse technique at oblique incidence. Vaughn Agy and Kenneth Davis.

Fields in electrically short ground systems: an experimental study. A. N. Smith and T. E. Devaney.

Diffraction of electromagnetic waves by smooth obstacles for grazing angles. James R. Wait and Alyce M. Conda.

Very-low-frequency radiation spectra of lightning discharges. W. L. Taylor and A. G. Jean.

Radio-wave scattering by tropospheric irregularities. Albert D. Wheelon.

Study at 1046 megacycles per second of the reflection coefficient of irregular terrain at grazing angles. Raymond E. McGavin and Leo J. Maloney.

Synoptic study of the vertical distribution of the radio refractive index. B. R. Bean, L. P. Riggs, and J. D. Horn.

\section{Journal of Research, Section D. Radio Propaga-} tion, Volume 63D, No. 3, November-December 1959. 70 cents.

Radio-refractive-index climate near the ground. B. R. Bean and J. D. Horn.

Path antenna gain in an exponential atmosphere. W. J. Hartman and R. E. Wilkerson (see abstracts above).

Effect of atmospheric horizontal inhomogeneity upon ray tracing. B. R. Bean and B. A. Cahoon.

Correlation of solar noise fluctuations in harmonically related bands. L. R. O. Storey.

A monochromatic low-latitude aurora. F. E. Roach and E. Marovich.

Pattern synthesis for slotted-cylinder antennas. James R. Wait and James Householder (see abstracts above).
Central Radio Propagation Laboratory exponential reference atmosphere. B. R. Bean and G. D. Thayer.

Excitation mechanisms of the oxygen 5577 emission in the upper atmosphere. E. 'Tandberg-Hanssen and F. E. Roach.

Method for measuring local electron density from an artificial satellite. L. R. O. Storey.

Thermodynamic properties of helium at low tempertures and high pressures, D. B. Mann and R. B. Stewart, TN8 (PB151367) \$1.25.

Transmission loss in radio propagation-II, K. A. Norton, TN12 (PB151371) \$3.00.

Technical considerations leading to an optimum allocation of radio frequencies in the band 25 to 60 Mc, K. A. Norton, TN13 (PB151372) \$2.50.

The role of government research laboratories, A. V. Astin, Elec. Engr. 78, No. 7, 738 (1959).

The ampere, F. B. Silsbee, Proc. IRE 47, No. 5, 643 (1959).

On the theory of reflection from a wire grid parallel to an interface between homogeneous media (II), J. R. Wait, Appl. Sci. Research 7, 355 (1959).

The basis of our measuring system, A. G. McNish, Proc. IRE 47, No. 5, 636 (1959).

Reflectors for a microwave Fabry-Perot interferometer, W. Culshaw, IRE Trans. on Microwave Theory and Techniques, Vol. MTT-7, No. 2, 221 (1959).

The research frontier, I. C. Schoonover, Saturday Rev. p. 58 (May 2, 1959).

The nature, cause and effect of the porosity in electrodeposits, III. Microscopic detection of porosity, F. Ogburn and D. W. Ernst, Plating 46, 831 (1959).

Paper test data from pendulum and inertialess testers, R. J. Capott, S. B. Newman, and J. Mandel, TAPPI 42, No. 6, 480 (1959).

Factors affecting the accuracy of distortion measurements made on the nodal slide optical bench, F. E. Washer and W. R. Darling, J. Opt. Soc. Am. 49, No. 6, 517 (1959).

Mechanism of contraction in the muscle fiber-ATP system, L. Mandelkern, A. S. Posner, A. F. Diorio, and K. Laki, Proc. Nat. Acad. Sci. 45, No. 6, 814 (1959).

Microwave spectrum of methyl germane, V. W. Laurie, J. Chem. Phys. 30, No. 5, 1210 (1959).

IGY instruction manual, Part 1: World days and communications, A. H. Shapley, Annals of International Geophys. Year 7, Pt. 1, 1 (Pergamon Press, New York, N.Y., 1959).

Measurement of ozone in terms of its optical absorption, R. Stair, Advances in Chem. Series of the Am. Chem. Soc., No. 21, 269 (1959).

On the perturbation of the vibrational equilibrium distribution of reactant molecules by chemical reactions, K. E. Shuler, 7th Symp. (International) on Combustion, London and Oxford, August 28 to September 3, 1958, Combustion Institute, p. 87 (Butterworths Sci. Pub., London, England, 1958). 
Rack for standard resistors, P. H. Lowrie, Jr., Rev. Sci. Instr. 30, No. 4, 291 (1959).

Spectroscopic evidence for triatomic nitrogen in solids at very low temperature, M. Peyron, E. M. Horl, H. W. Brown, and H. P. Broida, J. Chem. Phys. 30, No. 5, 1304 (1959).

The evaluation of small color differences: Part I. Visual observations, J. C. Richmond and W. N. Harrison, Am. Ceram. Bul. 38, No. 6, 292 (1959).
*Publications for which a price is indicated (except for Technical Notes) are available only from the Superintendent of Documents, U.S. Government Printing Office, Washington 25, D.C. (foreign postage, onefourth additional). Technical Notes are available only from the Office of Technical Services, U.S. Department of Commerce, Washington 25, D.C. (Order by PB number.) Reprints from outside journals and the NBS Journal of Research may often be obtained directly from the authors. 